\title{
Spatially Restricted Immune Responses Allow for Root Meristematic Activity During Bacterial Colonisation
}

\author{
Authors \\ Aurélia Emonet ${ }^{1}$, Feng Zhou ${ }^{1,4}$, Jordan Vacheron ${ }^{2}$, Clara Margot Heiman ${ }^{2}$, Valérie Dénervaud Tendon ${ }^{1}$, \\ Ka-Wai Ma ${ }^{3}$, Paul Schulze-Lefert ${ }^{3}$, Christoph Keel ${ }^{2}$, Niko Geldner ${ }^{1,5}{ }^{*}$
}

\author{
Affiliations \\ ${ }^{1}$ Department of Plant Molecular Biology, Biophore, UNIL-Sorge, University of Lausanne, 1015 \\ Lausanne, Switzerland \\ ${ }^{2}$ Department of Fundamental Microbiology, Biophore, UNIL-Sorge, University of Lausanne, 1015 \\ Lausanne, Switzerland \\ ${ }^{3}$ Max Planck Institute for Plant Breeding Research, 50829 Cologne, Germany \\ ${ }^{4}$ Present address: National Key Laboratory of Plant Molecular Genetics, CAS Center for Excellence in \\ Molecular Plant Sciences, Chinese Academy of Sciences, Shanghai 200032, China \\ ${ }^{5}$ Lead Contact \\ *Correspondence: niko.geldner@unil.ch (N.G.)
}

\section{Summary (150 words)}

Plants circumscribe microbe-associated molecular pattern (MAMP)-triggered immune responses to weak points of the roots. This spatially restricted immunity was suggested to avoid constitutive responses to rhizosphere microbiota. To demonstrate its relevance, we combined cell-type specific expression of the plant flagellin receptor (FLS2) with fluorescent defence markers and mapped immune competency at cellular resolution. Our analysis distinguishes cell-autonomous and non-cell autonomous responses and reveals lignification to be tissue-independent, contrasting cell-type specific suberisation. Importantly, our analysis divides the non-responsive meristem into a central zone refractory to FLS2 expression, and a cortex that becomes highly sensitised by FLS2 expression, causing meristem collapse upon MAMP exposure. Meristematic epidermal expression generates supercompetent lines that detect native bacterial flagellin and bypass the absence of response to commensals, providing a powerful tool for studying root immunity. Our precise manipulations and read-outs demonstrate incompatibility of meristematic activity and defence and the importance of cell-resolved studies of plant immunity. 


\section{Introduction}

2 Plant roots host a vast range of microorganisms in their rhizosphere. Amongst those, some can act as pathogens, negatively impacting plant growth and reproduction. However, the plant's sophisticated innate immune system keeps the vast majority of pathogens at bay. This MAMP-triggered immunity (MTI) rests on the recognition of highly conserved microbial molecules, recognised by plasma membrane-localised pattern-recognition receptors (PRRs) (Zipfel, 2008). One of the most investigated MAMPs is a 22 amino acid fragment of the bacterial flagellin protein (flg22). It is detected by the

FLAGELLIN SENSING 2 (FLS2) receptor (Felix et al., 1999; Gómez-Gómez and Boller, 2000; GómezGómez et al., 1999; Zipfel et al., 2004) and induces a signalling cascade including ROS production, calcium signalling, MAPKs (MITOGEN-ACTIVATED PROTEIN KINASE) phosphorylation and gene transcription, eventually leading to defence responses, such as callose and lignin deposition or phytoalexin production (Lee et al., 2019; Li et al., 2016).

Yet, plant PRRs equally perceive MAMPs from commensal or beneficial microbes, which are part of the normal plant rhizosphere. Whereas MTI is associated with growth inhibition (Chinchilla et al., 2007; Gómez-Gómez and Boller, 2000), a plethora of publications have established a growth promoting action of the soil microbiome (Berendsen et al., 2012). It therefore becomes particularly interesting to understand how roots accommodate a rhizosphere community, while avoiding a constant activation of PRRs and the growth-defence trade-off that comes with it. Many researchers have argued that the growth inhibition can be overcome by the ability of commensal microorganisms to supress plant immunity (Yu et al., 2019a). In addition, it was recently shown that the root has an inherently dampened MTI until it encounters damage, which locally boosts immune responsiveness (Zhou et al., 2020).

Indeed, root immune responses are generally lower than in the shoot, often because of an absence or low abundance of PRRs (Beck et al., 2014; Faulkner and Robatzek, 2012). Interestingly, plants restrict their defence to regions considered vulnerable. These coincide with regions where protective 
endodermal barriers are absent or broken, such as in the elongation zone and at the lateral root emergence sites. It is also where bacteria are found to preferentially accumulate (Beck et al., 2014; Bulgarelli et al., 2013; De Coninck et al., 2015; Faulkner and Robatzek, 2012; Millet et al., 2010; Poncini et al., 2017; Zhou et al., 2020).

Here, we set out to address the relevance of spatially limited responses. Wyrsch et al. (2015) ectopically expressed FLS2 under tissue-specific promoters and their data suggested that all root tissues were competent to mount an immune response provided that $F L S 2$ is expressed, although the nature of the tissue had a large influence on the strength of the innate immunity responses. Yet, the immune read-outs used in this work were at whole-plant or organ-level resolution and did not allow the authors to ascertain from which cell-type responses were originating, or whether responses were cell-autonomous, regional or systemic. Specifically, MAMP-induced ROS production, as well as cytosolic calcium increases, are known to act in a paracrine, even systemic fashion (Dubiella et al., 2013; Gilroy et al., 2014, 2016; Marhavý et al., 2019). Calcium waves were reported to initiate in the root elongation zone and to spread across tissues after flg22 treatment (Keinath et al., 2015; Stanley et al., 2018), opening the possibility that MAMP responses are induced in cell layers far away from the site of perception.

To address this issue, we combined new fluorescent markers lines with cell-type-specific FLS2 receptor lines. These marker lines use a triple mVenus fluorochrome coupled to a nuclear localisation signal (prom::NLS-3xmVenus). Combining concatemerisation with nuclear concentration generates high sensitivity and allows for a clear cellular assignment, not achievable with cytosolic, ER or PM-localised markers. These lines now enable us to observe damage and defence responses with cellular resolution, adding a crucial layer of complexity to our analyses (Marhavý et al., 2019; Poncini et al., 2017; Vermeer et al., 2014; Zhou et al., 2020). We also added fluorescence-based markers that have been used for assessing cytosolic calcium changes triggered by flg22 at single cell resolution (Thor and Peiter, 2014). 
This has allowed us to manipulate and quantitatively map defence responses at cellular resolution in the root. Our approach revealed the presence of regions refractory to FLS2 presence, as well as others which are super-competent. We show that inappropriate FLS2 expression has drastic impact on root development, affecting growth, cell wall composition and cell viability. To assess the impact of $F L S 2$ misexpression in response to natural microbiota, we use our super-competent lines in the presence of commensal bacteria, normally not detected by wild-type plants. We demonstrate stimulation of FLS2 directly by native, bacteria-derived flagellin and reveal the importance of spatial restriction of immune responses in order to adequately balance growth and defence.

\section{Results}

\section{Tissue-specific expression of FLS2}

In order to analyse the ability of the different root tissues to respond to flg22, we used lines expressing FLS2 under cell-type-specific promoters in an fls2 mutant background (Wyrsch et al., 2015). We selected lines expressing FLS2-GFP driven by three different tissue-specific promoters: WEREWOLF for epidermis (WER::FLS2), CASPARIAN STRIP DOMAIN PROTEIN 1 for endodermis (CASP1::FLS2), and SHORT-ROOT for inner cell layers (SHR::FLS2). As controls, we monitored FLS2-GFP driven by the constitutive promoter UBIQUITIN 10 (UBQ10::FLS2) and by the native FLS2 promoter (FLS2::FLS2). As described previously, endogenous FLS2 expression was observed principally in the differentiated stele (Beck et al., 2014) (Fig.1A) but also weakly in all tissues from the elongation to the differentiated zone, as well as in root cap cells (Zhou et al., 2020) (Fig.1B). WER::FLS2, by contrast, was strongly expressed in the epidermis of the meristematic zone (Fig.1), as predicted (Lee and Schiefelbein, 1999), with some weak signal in the older cortex (elongation zone) (Fig.S1A). In agreement with its established expression (Benfey et al., 1993; Helariutta et al., 2000), we detected SHR::FLS2 in the stele close to the meristem (Fig.1), but also faintly in the neighbouring endodermis, suggesting that either FLS2 proteins or mRNAs move through plasmodesmata (Fig.S1D). CASP1::FLS2 had the predicted exclusive 
expression in differentiated endodermis (Fig.1, S1B) and UB10::FLS2 was detected in all tissues throughout the root, from meristem to differentiation zone (Fig.1, S1C).

\section{Ectopic FLS2 expression alters MTI response patterns}

We crossed our selection of FLS2 lines with two typical MTI transcriptional markers, PEROXIDASE 5 (PER5) and MYB DOMAIN PROTEIN 51 (MYB51), and generated homozygous lines at all three loci (marker, prom::FLS2 and fls2). As control, we used the two markers in wild-type Col-0 background. Markers were chosen for their strong response to flg22 and their divergent response patterns (Poncini et al., 2017; Wyrsch et al., 2015; Zhou et al., 2020). In addition, we developed a pipeline using tissuespecific quantitative analysis, for measuring and comparing MTI responses in an unbiased fashion (Fig.

S2). For this, we additionally introduced ubiquitous nuclear markers (UBQ10::NLS-mTurquoise or UBQ10::NLS-tdTomato) in all our genotypes, which allows to call all nuclei as separate, individual 3D Regions-of-Interests (ROIs), even those with weak or absent MTI-response. After mock or flg22 treatment and fixation, cell-wall-stained roots were imaged at three different zones of the root: Meristem (MZ), Elongation (EZ) and Differentiation (DZ). Each nucleus was automatically detected as a 3D object and the obtained nuclei object maps were then combined to the cell wall marker channels to manually curate and assign each nucleus to a tissue. Once the selected nuclei were assigned, mean intensity for each cell type per zone per treatment per genotype were calculated and colour coded for the generation of a quantitative MTI-response atlas for each prom::FLS2 line (Fig.S2, values in Fig.S4).

Our cell-specific quantification and microscopic analysis confirmed that PER5 is not expressed in absence of flg22 treatment (Fig.2A, 2BC), but that MYB51 presents a basal, flg22-independent expression in the epidermis and root cap cells of the undifferentiated tissues (MZ and EZ) and in the stele and the cortex of the DZ (Fig.S3A, S3C). In wild-type plants, both MAMP markers are strongly induced in the EZ, recapitulating previous observations (Fig.2A and S3A) (Millet et al., 2010; Poncini et al., 2017; Zhou et al., 2020). Specifically, PER5 is triggered almost exclusively in the elongating epidermis and root cap cells (Fig.2B, 2C, 2D). MYB51 induction is restricted to these same tissues close to the meristem, but induction expands to cortex and pericycle cells in the later root (Fig.S3C, S3D). 
For both markers, changing expression of $F L S 2$ had an obvious impact on the pattern of responses.

101

102

103

104

105

106

107

108

109

110

111

112

113

114

115

116

117

Rather than remaining restricted to the elongation zone, PER5 and MYB51 induction largely follows the ectopic FLS2 expression pattern. The defence markers extend to the whole root in UBQ10::FLS2, while they are restricted to the DZ or the MZ in CASP1::FLS2 and WER::FLS2, respectively (Fig.2A and S3A). As expected, the fls 2 mutant does not respond to flg22 in any tissue.

PER5 responds only in the differentiated endodermis in the CASP1::FLS2 recombinant line, which matches with the very specific expression pattern of CASP1 promoter. For WER::FLS2 line, the PER5 response also follows $F L S 2$ expression. We could quantify a strong response in root cap cells and the meristematic epidermis, extending until the early DZ, as well as in cortex cells, where we could also detect FLS2 protein (Fig.2B, 2C, 2D, S1A). In contrast to PER5, we detected MYB51 response to flg22 not only in cells expressing FLS2, but also some degree of induction in neighbouring cells (Fig. S3B, S3C, S3D). Intensity ratio between flg22 and mock treated plants were calculated and represented graphically in Fig.S5. Non-cell-autonomous responses were obvious for MYB51 in the DZ of CASP1::FLS2. Although FLS2 is specifically expressed in the endodermis, we could barely detect any MYB51 responses in this tissue, while the neighbouring stele and cortex cells strongly up-regulated MYB51 (Fig.S3C, S3D, S5). Similarly, flg22 treatment led to MYB51 expression not only in the epidermis and cortex, but also in central tissues in WER::FLS2. Thus, we concluded that MYB51 induction by MAMPs is controlled by non-cell autonomous mechanisms, in contrast with the strict cell-autonomy of PER5 and FRK1 (this work and Zhou et al., 2020).

\section{FLS2 expression is insufficient to cause flg22-responses in the vascular meristem}

Intriguingly, some tissues were also completely refractory to flg22-triggered responses. Despite a clear presence of FLS2 in the vascular meristem (Fig.1B), flg22 treatment did not trigger PER5 or MYB51 in SHR and UBQ10::FLS2 lines (Fig.2, S3), except for some weak MYB51 induction in meristematic pericycle cells in UBQ10::FLS2 (Fig.S3D, S5). We conclude that flg22-induction of MYB51 in the pericycle is due to a non-cell autonomous signal from outer cell layers. Thus, central meristematic tissues differ from outer tissue layers in their competence to respond to flg22 in the presence of receptor. 
127 Cytosolic $\mathrm{Ca}^{2+}$ increases are among the earliest responses upon MAMP perception, preceding 128 transcriptional changes (Jeworutzki et al., 2010; Seybold et al., 2014). In roots, Ca ${ }^{2+}$ influx after flg22 perception was shown to spread across tissues (Keinath et al., 2015). However, since many cells express some degree of FLS2 in wild-type, it is impossible to dissect to what extent such waves represent a non-cell autonomous propagation of the $\mathrm{Ca}^{2+}$ signalling, or are due to flg22 diffusion and direct stimulation of the different tissue layers and regions. We therefore introduced the intensitybased $\mathrm{Ca}^{2+}$ reporter $\mathrm{R}$-GECO1 in our transgenic lines (Keinath et al., 2015). We observed in WER::FLS2that, like in WT (Movie 1 and 6), calcium signals initiate in the epidermis and spread to inner tissues (Fig.3AB, Movies 2 and 7). Since the receptor was not expressed in central tissues, this clearly demonstrates the non-cell autonomous nature of FLS2-stimulated calcium signalling. This spreading of $\mathrm{Ca}^{2+}$ could be observed in all recombinant lines tested, with the intriguing feature that wave direction could be manipulated - i.e. in both CASP1::FLS2 and SHR::FLS2 lines, the wave started first in the endodermis then spread to outer and inner tissues (Fig.3CD, Movie 8 and 9). Moreover, in these two lines, the wave starts in the differentiated zone rather than in the elongation zone (Movies 3 and 4). When FLS2 was expressed in all tissues under UBQ10 promoter, all tissues respond almost simultaneously (Fig.3E, Movie 5 and 10). Taken together, while transcriptional read-outs are largely cell-autonomous, with some degree of tissue-specificity, cytosolic calcium increases represent a noncell autonomous signalling branch. This implies that even cells that are neither exposed to MAMPs, nor possessing perception capacity, are nonetheless rapidly receiving some sort of stress signal in the form of a calcium wave.

\section{Epidermal meristematic expression of FLS2 leads to flg22 hypersensitivity and meristem collapse}


UBQ10::FLS2 line was strongly reduced with additionally stunted shoot development (Fig.4A and 4B).

More surprisingly, a strong root length inhibition was also observed in the WER::FLS2 line, although this lines expresses FLS2 only in young epidermal and root cap cells. SHR::FLS2 and CASP1::FLS2, by contrast, showed root growth similar to wild-type.

In order to precisely identify the tissue responsible for root growth inhibition, we generated two additional prom::FLS2 lines using the RCH1 (RECOGNITION OF C.HIGGINSIANUM) and PRP3 (PROLINERICH PROTEIN 3) promoters (Marquès-Bueno et al., 2015). RCH1 is expressed in the whole meristem, while PRP3 is expressed strongly in differentiating root hair cells (Fig.S1E). While PER5 induction followed the expression of FLS2 in both lines (Fig. S1GH), only RCH1::FLS2 presents an increased root growth inhibition (Fig.S1F), whereas PRP3::FLS2 responds as wild-type (Fig.S1I). Therefore, we conclude that it is the expression of $F L S 2$ in meristematic epidermal cell layers that causes hypersensitive root growth inhibition in response to flg22. Indeed, when comparing the pattern of PER5 expression between wild-type and WER::FLS2 at high resolution, it is evident that only the meristematic epidermal cells show strong PER5 induction in WER::FLS2, whereas root cap cells show flg22 responsiveness in both lines. This suggests that MTI in epidermal cells are the cause of supercompetent response (Fig. 4C). Importantly, neither the MAMPs elf18, chitin or the LPS fragment 3-OHC10:0, nor Atpep1 showed enhanced PER5 in WER::FLS2 (Fig.4D). This demonstrates that ectopic FLS2 expression does not cause a global upregulation of responsiveness to MAMPs, but specifically affect flg22 signalling.

Interestingly, treatment of WER::FLS2 super-competent line with flg22 induces profound morphological changes in the root, not observed in wild-type. After two days of treatment, cells reaching the transition zone start to swell and division patterns become disorganized, giving rise to bulky meristem shapes (Fig.4E, upper panel, 4G). Virtual cross-sections revealed that cortex cells expand tremendously, dislocating epidermal cells (Fig.4F). Thus, precise spatial regulation of $F L S 2$ expression levels is necessary to avoid severe growth inhibition caused by flg22-induced disorganized cell expansion in the meristem. 
MTI is known to modify cell wall composition, such as callose deposition or lignification (Chezem et al., 2017; Lee et al., 2019; Millet et al., 2010). Indeed, lignin or suberin deposition are long-known damage and immune responses (Bernards, 2002; Hijwegen, 1963; Kamula et al., 1994; Messner and Boll, 1993; Ranathunge et al., 2008; Thomas et al., 2007), but have not been widely adopted in modern studies on MTI (Lange et al., 1995; Mandal and Mitra, 2007), see (Chezem et al., 2017; Lee et al., 2019) for exceptions.

Interestingly, we found that flg22 treatment induced strong lignification from transition to differentiated zone in WER::FLS2 (Fig. 4E and S6A). Lignin was deposited between epidermis and cortex cells, mainly at the corners (Fig. 4F). In younger regions, lignin was also found between epidermis and root cap cells. All other recombinant lines also showed lignin deposition following their respective $F L S 2$ expression pattern, except in the stele, matching the absence of PER5 response in these tissues (Fig.S6B). Interestingly, no lignin deposition could be observed in flg22-treated wild-type roots (Fig.4E, S6), fitting with previous reports (Chezem et al. (2017). It is intriguing to speculate that PER5, ROSproduction and other flg22-responsive genes, categorised as "oxidative stress" response genes (Tognolli et al., 2002), are actually part of a lignification response that stays below a productive threshold in wild-type, but pivots into a full lignification upon flg22-stimulation of FLS2 overexpression lines.

The stronger root growth inhibition observed in the super-competent WER::FLS2 line could be due to

197 the impact of lignin deposition in the transition zone. To test if cell wall reinforcement by lignin 198 prevents cell division and elongation, we inhibited lignin formation with the monolignol synthesis 199 inhibitor piperonylic acid (PA), expecting to restore root growth (Fig. 4I). Nevertheless, even in the absence of lignin, WER::FLS2 still showed root meristem collapse and stronger RGI than wild-type (Fig. $4 \mathrm{H})$. 
Ectopic lignin deposition occurs in the endodermis as a compensatory mechanism for deficient

We wanted to assess whether the overactivation of MTI could trigger the deposition of suberin in cells expressing FLS2. In wild-type untreated plants, suberin is usually present in the endodermis only, suberized zone (Andersen et al., 2015, 2018).

In wild-type, suberin was not induced by flg22 (Fig.5AB). In contrast, lines expressing FLS2 in the endodermis, such as CASP1::, SHR:: and UBQ10::FLS2, showed increased endodermal suberization, leading to a complete disappearance of the patchy zone (Fig. 5B). Earlier suberisation is not simply due to earlier differentiation of endodermal cells due to growth arrest, since WER::FLS2 still conserved a normal proportion of patchy and suberized zone despite its shorter root length. Reversely, CASP1:: and SHR::FLS2 root growth was not affected by flg22, but suberin formed nevertheless much earlier. Therefore, flg22 can induce suberization only when expressed in the endodermis. This endodermisspecific suberisation is a nice demonstration of a flg22 response that only occurs in a specific cellular context.

\section{Super-competent WER::FLS2 line can detect native bacterial flagellin}

The strong impact of flg22 on WER::FLS2 root growth and cell wall modification prompted us to evaluate whether commensal bacteria would have a similar effects. Indeed, plants that mount ectopic defences in sensitive tissues might suffer from the presence of usually harmless bacteria and tip the balance between growth and defence. The model commensal/beneficial Pseudomonas protegens CHAO does not induce MTI responses in wild-type plants, except at high concentration or if the root is wounded (Zhou et al., 2020). However, when inoculated on WER::FLS2 line, a very evident PER5 induction could be observed, although no synthetic flg22 peptide was added (Fig.6A). This experiment

227 is therefore a first clear example, where a flg22 response is caused by actual, living bacteria. This 228 flagellin must be released and processed into FLS2-binding smaller peptides (Buscaill et al., 2019). 
To confirm that the induction of PER5 was caused by native, bacterial flg22, we infected seedlings with demonstrating that defences are induced by the direct FLS2-mediated detection of bacteria-derived flagellin molecules (Fig.6A). We then assessed the impact of CHAO bacteria on root growth. Surprisingly, despite its induction of PER5, CHAO did not significantly enhance root growth inhibition in WER::FLS2 compared to wild-type (Fig.6B). One explanation would be that some commensal bacteria are able to attenuate the excessive MAMP-triggered immune responses in WER::FLS2, thus avoiding root growth inhibition and deleterious defence responses (Garrido-Oter et al., 2018; Pel and Pieterse, 2013). Indeed, Ma et al. were unable to observe any growth phenotype of WER::FLS2 plants grown in non-sterile soil. Interestingly, they reported that $41 \%$ of root commensals can suppress

MAMP-triggered root growth inhibition in mono-associations (manuscript in preparation).

\section{Bacterial community members have diverse impact on WER::FLS2}

243 In order to obtain a more comprehensive picture of how WER::FLS2 affects responses to bacteria, we

244 screened a set of 34 bacterial isolates from the At-SPHERE culture collection of Cologne (Bai et al., 245 2015) for both induction of PER5 marker and enhanced root growth inhibition in WER::FLS2 compared to wild-type lines. We selected isolates to represent bacteria from all phyla, with a bias towards bacteria predicted to possess a flg22 peptide sequence recognised by the FLS2 receptor (Fig.6C, Table S1) (Garrido-Oter et al., 2018). Amongst the 17 strains predicted to be recognized by FLS2 based on their sequence, only 10 triggered an enhanced PER5 marker induction in WER::FLS2. Moreover, five additional strains, without a predicted recognizable flg22 sequence, had the same effect. This plant. 
Although half of bacterial isolates could induce PER 5 marker specifically in the WER::FLS2 line, only 5 of them affected WER::FLS2 root growth more strongly than WT, though often with great variation (Fig.6C, Table S1). However, Pseudomonas isolate R569 caused strongly enhanced PER5 induction (Fig.6D) and root growth inhibition compared to WT (Fig.6E). This effect was very robust and was repeatedly observed both in Lausanne and Cologne laboratory growth conditions (Fig.S7C). We demonstrated that commercial, synthetized flg22 from Pseudomonas aeruginosa as well as from isolate R569 (flg22 $2_{\text {R569) }}$ similarly induced PER5 marker expression and inhibited root growth. These effects were abrogated in the fls2 mutant background (Fig.S7B). We conclude that the commensal R569 isolate induces MTI responses in the WER::FLS2 line through its native flg22 peptide, which then causes an unbalancing of growth and defence not observed when the bacterium grows on wild-type roots.

\section{Discussion}

It is not understood why only a restricted subset of root tissues can directly respond to MAMPs in the absence of other stimuli (Millet et al., 2010; Poncini et al., 2017; Zhou et al., 2020). The combination of tissue-specific receptor expression and cellular resolution read-outs presented here provides insights into the consequences of altering the spatial patterns of MTI in roots. Our work reveals three important features of MAMP responses.

First, different MTI responses are highly tissue-specific and varying in cell-autonomy. Suberin, for example, is only induced in the endodermis. While PER5 induction is strictly cell autonomous, MYB51 and calcium signals are found in cell lacking FLS2 receptor. It will be important to describe larger numbers of response genes for a comprehensive view of MTI. Cell-type specific transcriptomic analysis can complete our understanding of tissue-specific immune pathways (Rich-Griffin et al., 2020). Our prom::FLS2 lines coupled to transcriptional read-outs can now help to distinguish cell-autonomous responses from indirect activation by MTI. 
Secondly, we found that the vascular meristem is refractory to flg22 even when expressing FLS2 receptor. The seemingly contradictory finding in Wyrsch et al. (2015) can be explained by the wholeorgan read-outs used, as well as use of $L B D 16:: F L S 2$, thought to be stele-specific, but that we found to also slightly express in other tissues (Fig.S1J). Lack of downstream signalling components or increased activity of negative regulators could both be responsible for the stele's inability to respond to flg22. The vascular meristem might be particularly vulnerable to an activation of defence as it contains earlydifferentiating phloem providing nutrition and hormones to the growing meristem.

Finally, we observed root regions that can be rendered super-competent by FLS2 expression. We speculate that epidermal meristematic cells are not responsive in wild-type (Millet et al., 2010; Zhou et al., 2020), because only the outer root cap cells can mount MAMP responses that are not detrimental to meristem function. This might be linked to the particular fate of root cap cells that enter apoptosis once they reach the transition zone (Fendrych et al., 2014; Kumpf and Nowack, 2015) and excrete mucilage and secondary metabolites influencing root microbiota (Bulgarelli et al., 2013; Kumpf and Nowack, 2015). By contrast, epidermal cells might only maintain a competency to respond, if root cap damages by pathogens or other stresses induce FLS2 expression. Indeed, we clearly showed that constitutive expression of $F L S 2$ in the meristematic epidermis leads to drastic changes in the root structure upon flg22 treatment in ways that could be detrimental to growth.

Though lignification upon actual bacterial infection is well documented (Lee et al., 2019; Nicholson and Hammerschmidt, 1992; Vance et al., 1980), treatment with single MAMP was rarely seen to stimulate root lignin deposition (Adams-Phillips et al., 2010; Chezem et al., 2017; Robertsen, 1986; Smit and Dubery, 1997). Here we show that strong FLS2 expression reveals the capacity of MTI responses to modify cell walls, probably overriding endogenous negative feedbacks that prevent this from happening in wild-type. This provides an opportunity to study MTI-induced lignification in a simplified and reproducible setting. Interestingly, ectopic corner lignification together with defence genes induction are also observed in response to CIF2 peptide treatment in the endodermis (Alassimone et al., 2016; Doblas et al., 2017; Fujita et al., 2020, 2020; Pfister et al., 2014), suggesting the 

is only partly explaining the severe root growth inhibition we observe. Other factors produced in response to flg22 might also interfere with meristem function, such as basic coumarins (Stringlis et al., 2019), which inhibit cellulose, resulting in meristem swelling similar to the one observed on WER::FLS2 (Hara et al., 1973).

Our work also reveals that overexpression of a single PRR in a competent, but otherwise nonresponsive cell-type, bypasses the absence of visual immune responses to commensal bacteria (Garrido-Oter et al., 2018; Millet et al., 2010; Yu et al., 2019b; Zhou et al., 2020). Though bacteria can also inhibit MTI (Couto and Zipfel, 2016; Yu et al., 2019a), MAMPs produced by rhizosphere bacteria might often be too low in concentration to activate MTI responses in the first place. Therefore, roots might appear largely unresponsive to bacterial presence without additional stresses (Zhou et al., 2020). The obvious root growth phenotype triggered by MTI in WER::FLS2 lines proves to be a powerful tool to investigate the effect of commensals on root immune responses. Our super-competent lines have allowed for the first time to directly observe stimulation of FLS2 by a native flagellin peptide from an avirulent bacterium. Generally, the cocktail of elicitors that bacteria are thought to release prevent assignment of a MAMP response to an individual MAMP (Tang et al., 2017). The WER::FLS2 line now generates a cell type that responds only to a single MAMP and can test predictions about flg22 peptide detectability, release and processing. Extending our approach, the ectopic overexpression of potential PRR receptors in the epidermal meristem cells could be used to functionally pair novel receptors and ligands.

It has become evident that immune responses cannot be understood without taking into consideration the specificities of different cell type and developmental stages. Our data establishes the necessity for the plant to spatially restrict its immune response. This spatial allocation of defence capacities might in turn influence the microbial colonization pattern of the rhizosphere. The new tools presented will pave the way for a better understanding of bacterial community structures in roots. 


\section{Acknowledgement}

331

332

333

334

335 (ALTF 1139-2014).

\section{Author contributions}

A.E. and N.G. conceived, designed, and coordinated the project. A.E., F.Z., J.V., C.M.H., V.D.T. and N.G. revised the manuscript and were involved in the discussion of the work.

\section{Declaration of interests}

The authors declare no competing interests. 


\section{Material and methods}

349

350

351

352

353

354

355

356

357

358

359

360

361

362

363

364

365

366

367

368

369

370

371

\section{Plant material}

Arabidopsis thaliana ecotype Columbia Col-0 was used for most experiments. The T-DNA line FLS2 was obtained from NASC (SALK_062054C) and originally described in (Zipfel et al., 2004). The recombinant WER::FLS2-3myc-GFP, CASP1::FLS2-3myc-GFP, SHR::FLS2-3myc-GFP, UBQ10::FLS2-3myc-GFP, LBD16::FLS2-3myc-GFP in fls2 (SAIL691_C04) background, as well as FLS2::FLS2-3myc-GFP in Wassilewskija Ws-0 background were provided by Prof. Thomas Boller's group (Robatzek et al., 2006; Wyrsch et al., 2015). The defence marker lines PER5::NLS-3mVenus and MYB51::NLS-3mVenus are described in (Poncini et al., 2017). Calcium signalling analysis was carried out thanks to the line UBQ10::R-GECO1 kindly shared by Prof. Melanie Krebs's group (Keinath et al., 2015).

PER5::NLS-3mVenus and MYB51::NLS-3mVenus lines were crossed to the four recombinant lines WER::, CASP1::, SHR:: and UBQ10::FLS2-3myc-GFP fls2 lines as well as to the fls2 mutant. In addition, UBQ10::R-GECO1 was first crossed to fls2 mutant, then the resulting homozygous line was crossed again to the four recombinant lines (WER::/CASP1::/SHR::/UBQ10::FLS2-3myc-GFP fls2), so that F1 could be directly used for experiments. For quantification of tissue-specific nuclear signal, the constructs UBQ10::NLS-mTurquoise or UBQ10::NLS-tdTomato were transformed by floral dipping method in all PER5::/MYB51::NLS-3mVenus marked prom::FLS2-3myc-GFP fls2, fls2 and wild-type lines (Clough and Bent, 1998). In addition, RCH1::FLS2-GFP, PRP3::FLS2-GFP and GRP::FLS2-GFP were transformed in fls2 (SALK_062054C).

\section{Plant growth conditions}

For all experiments, seeds were surface-sterilized by gaseous chlorine for 2.5 hours or immersed in a solution of $70 \% \mathrm{EtOH} 0.01 \%$ Triton-X-100 for $5 \mathrm{~min}$, washed once in $96 \% \mathrm{EtOH}$ and dried under sterile conditions. Seeds were stratified in the obscurity for 2 days, then germinated on $1 \%$ agar plates containing half-strength Murashige and Skoog ( $1 \frac{1}{2} \mathrm{MS}$ ) medium and 500mg/l MES (Duchefa). Seedlings 
372 were grown vertically for 5 days before analysis (otherwise differently specified) in continuous light at $37323^{\circ} \mathrm{C}$.

374 For experiments done in Cologne, seeds were sown on $1 \%$ Bacto-Agar supplemented with $1 / 2$ MS with $375250 \mathrm{mg} / \mathrm{I}$ of MES. Seedlings were grown in a light cabinet with short day conditions (10h light-14h dark, $21^{\circ} / 19^{\circ} \mathrm{C}, 65 \%$ relative humidity).

\section{Bacterial strains and growth conditions}

Pseudomonas protegens strain $\mathrm{CHAO}$ used in this study is a tobacco root isolate with plant-beneficial activities (Stutz et al., 1986). CHAO mutants $\Delta$ fliC and $\Delta$ fleQ carrying in-frame deletions in the fliC and fleQ genes, respectively, were generated using the suicide vector PEMG and the I-Scel system (Kupferschmied, 2015; Martínez-García and Lorenzo, 2011) adapted to P. protegens (Kupferschmied et al., 2014) with plasmids and primers listed in Supplemental Table S2. The Pseudomonas R569 and other natural commensal bacterial isolates were obtained from the At-SPHERE culture collection (Bai et al., 2015). CHAO strains and commensal isolates were routinely cultured at $28^{\circ} \mathrm{C}$ in, respectively, lysogeny broth (LB) medium ( $1 \%$ tryptone, $0.5 \%$ yeast extract and $1 \% \mathrm{NaCl}$ ) or half-strength tryptic soy broth (TSB) (Sigma-Aldrich).

\section{Plant plasmid construction}

Generation of expression constructs was performed with both In-Fusion Advantage PCR Cloning Kit (Clontech) and Gateway Cloning Technology (Invitrogen). selection. For the red version of nuclei labelling, the plasmid UBQ10::NLS-tdTomato was used for its 
reaction. pDONR P1-NLS-tdTomato-P2 was previously generated using in-Fusion cloning to integrate the NLS sequence to pDONR P1-tdTomato-P2.

RCH1::FLS2-GFP, PRP3::FLS2-GFP and GRP::FLS2-GFP were generated combining the respective entry clones pDONR L4-pRCH1-R1 and L4-pPRP3-R1 (SWELL lines)(Marquès-Bueno et al., 2015), or pDONR L4-pGRP-R1 (Andersen et al., 2018), with pDon207 containing the L1-FLS2-3xmyc-GFP-L2 sequence (Wyrsch et al., 2015), in the destination clone pH7m24GW,3.

\section{Elicitors and inhibitors treatments}

Commercial flg22 $\mathrm{Pa}$ peptide from Pseudomonas aeruginosa (QRLSTGSRINSAKDDAAGLQIA) was

ordered from EZBioLab. Elf18 oligopeptide from Escherichia coli strain Gl826 (Acdissolved in absolute $\mathrm{EtOH}$ at a concentration of $20 \mathrm{mM}$ for stock solution. indicated concentration. Seedlings were grown vertically for 4 days on small $1 / 2 \mathrm{MS}$ petri dishes $(5.5 \mathrm{~cm}$ diameter), then $1.5 \mathrm{ml}$ of elicitor solution was gently poured over the seedlings to avoid damages induced by transfer. Care was taken that all roots were properly submersed. Seedlings were incubated horizontally for $24 \mathrm{~h}$ before live imaging analysis of 5-day-old plants or fixation.

For root growth analysis, 5day-old seedlings were carefully transferred on new $1 / 2$ MS agar plates containing $1 \mu \mathrm{M}$ flg22 $\mathrm{pa}$ or flg22 $2_{\mathrm{R} 569}$ and grown vertically for 6 days in standard growth conditions. 
422

To overcome PA degradation by light but still conserve proper root growth in control conditions, plates were inserted in black boxes open to the top, allowing roots to grow in the obscurity but leaves to reach the light. Roots were hidden from top light using black sterile plastic caches. After overnight pretreatment, seedlings were again transferred on plates containing $10 \mu \mathrm{M} \mathrm{PA} / \mathrm{EtOH}$ with/without $1 \mu \mathrm{M}$ flg22/mock, using the same black boxes. Their root tip location was recorded. $48 \mathrm{~h}$ after the first transfer, root growth was measured and seedlings were fixed as described.

\section{Microscopy settings and image processing}

Imaging was performed on either a Zeiss LSM880, LSM700 or a Leica SP8 inverted confocal scanning microscope. Pictures were taken with a 63x oil immersion objective (Zeiss LSM880), 63x water immersion objective (Leica SP8), 40x water immersion objective (Leica SP8), as well as 20x or 10x dry immersion objectives for tile-scan with $10 \%$ overlap (Zeiss LSM880 or LSM700).

The excitation and detection windows were set as following: for visualisation of FLS2 and defence markers, on Leica SP8, GFP/PI (488nm, 500-530nm and 600-670nm); GFP/mVenus/PI (488nm, 490508nm; 514nm, 517-560nm and 600-670nm, sequential scan), on Zeiss LSM880, GFP/PI(488nm, 500530nm and 600-650nm respectively), mVenus $(514 \mathrm{~nm}, 520-550 \mathrm{~nm})$. For lignin analysis: on Zeiss LSM880, calcofluor $(405 \mathrm{~nm}, 425-475 \mathrm{~nm})$, basic fuchsin $(561 \mathrm{~nm}, 600-650 \mathrm{~nm})$. For cell-specific quantification: on Zeiss LSM880, DirectRed 23/mVenus/mTurquoise2 (561nm, 580-700nm; 514nm, 520-590nm; 458nm, 460-500nm; sequential scan) and Calcofluor/mVenus/tdTomato (405nm, 415450nm; 514nm, 520-545nm; 561nm; 570-640nm, sequential scan). For calcium analysis: Zeiss LSM880, R-GECO1 (561nm, 580-640nm). For suberin staining: on Zeiss LSM700, fluorol yellow $(488 \mathrm{~nm}, 500-$ $600 \mathrm{~nm})$.

Images were processed using the Fiji software. For cross-section maximum projection of MAMPinduced signal (Fig.2B, 2C, S3B, S3C), z-stack pictures were resliced then realigned thanks to the Descriptor-based series registration $(2 d / 3 d+t)$ plugin. A maximum projection of the MAMP marker channel was then merged to a representative single stack of the PI-stained cell wall channel. 


\section{Fixation and staining}

448 Fixation and cell-wall staining were performed according to adapted Clearsee protocol (Kurihara et al., 449 2015; Ursache et al., 2018). Briefly, 5-day-old seedlings were fixed for $1 \mathrm{~h}$ at room temperature under vacuum in 4\% paraformaldehyde PBS solution, using 6-well plates, then washed twice for $1 \mathrm{~min}$ with PBS. Once fixed, seedlings were cleared in Clearsee solution for at least $24 \mathrm{~h}$ under mild shaking. To visualize cell wall for quantification, clearing solution was exchanged with either $0.1 \%$ Calcofluor White or $0.1 \%$ Direct Red 23 in Clearsee solution. After at least respectively $30 \mathrm{~min}$ and $2 \mathrm{~h}$ of staining, the staining solution was removed and samples rinsed once in fresh Clearsee solution, then washed for $30 \mathrm{~min}$ in a renewed Clearsee solution with gentle shaking before mounting.

For combined cell wall and lignin staining, fixed and cleared samples were incubated overnight in a Clearsee solution supplemented with 0.2\% Basic Fuchsin and 0.1\% Calcofluor White. Once the dye solution removed, samples were rinsed once, washed firstly $30 \mathrm{~min}$ then at least $1.5 \mathrm{~h}$ before observation.

\section{Cell-specific quantification}

461 To realize the complete atlas of defence marker responses, the different prom::FLS2 lines analysed were first transformed with UBQ10::nIs-mTurquoise2 to delimit nuclei. Alternatively, wild-type PER5:: and MYB51::NLS-3Venus lines were transformed with UBQ10::nls-tdTomato, which comprise a FastRed rather than a Kanamycin selection. This allowed to quantify directly the T1 and skip one generation. After flg22 treatment, seedlings were fixed in Clearsee and their cell wall stained with DirectRed23, or Calcofluor White respectively. Z-stack were imaged on half section of the roots at 4 different positions, i.e. meristematic zone (MZ), elongation zone (EZ), 12 cells and 30 cells after the onset of elongation for 3 to 6 roots by treatment (mock and flg22) and by genotype. Three channels Calcofluor White) and the defence markers PER5 and MYB51 (mVenus), using the same settings on all

471 pictures for mVenus channel. However, wild-type UBQ10::NLS-tdTomato and prom::FLS2 UBQ10::NLS- 
constructs. Pictures were processed on Fili software with a custom batch macro automatizing the following pipeline (Schneider et al., 2012). Images were first resliced from the top, then the three channels were separated. A Gaussian blur was applied on the nuclear and cell wall marker channels, while the PTI marker channel was left untouched to not affect the signal to measure. In a second step, the cell wall channel was subtracted to the nuclear channel to reduce the unspecific background noise of the UBQ10::nIs-mTurquoise2 marker. The "cleaned" nuclear marker channel was transform to 8 bits to facilitate further processing.

We then used the 3D suite to generate a 3-dimensional Region Of Interest (ROI) for each nucleus (Ollion et al., 2013). We first applied the plugin 3D iterative thresholding on the 8bits-cleaned-nuclear marker channel (Gul-Mohammed et al., 2014). In this process, all possible thresholds are tested, which will detect objects for all thresholds. Subsequently, the algorithm will define the best object segmentation for each of the object, which means that different objects can be segmented with different threshold. This is particularly useful to detect objects with variable intensity in an uneven background, to which a single intensity threshold would either miss many objects or include background noise. We used the following settings: $\min$ vol pix $=250, \max$ vol pix $=10000, \min$ threshold $=0$, min contrast $(\exp )=5$, criteria method $=$ COMPACTNESS, threshold method $=$ STEP, Segment results $=$ All, value method $=10.0$, Starts at mean $=$ on. The plugin gives as output the 3D threshold delimiting all the future ROIs, i.e. the nuclei to quantify. It must be noted that depending on the pictures, some nuclei can be missed, or false positive can be added, but all pictures were then manually curated in a later step. The output came as 2-channels-images, whose last channel is completely black and can be removed by the splitting channel function.

We then use the 3D object counter plugin to define all ROls, based on the 3D threshold obtained previously, and to redirect the analysis on the defence marker channel (Bolte and Cordelières, 2006). Options were set using the 3D OC Options as following: all parameters were selected, i.e. "Volume", "Nb of Obj. voxels", "Nb of Surf. voxels ", "Integrated Density", "Mean Gray Value", "Std Dev Gray Value", "Median Gray Value", "Minimum Gray Value", "Maximum Gray Value", "Centroid" "Mean 
distance to surface", "Std Dev distance to surface", "Median distance to surface", "Centre of mass", and "Bounding box". In addition, we ticked both parameters "Close original images while processing" and 'Show masked image. The maps' parameters were set as follows: dots size $=5$, font size $=12$, "Show numbers" and "White numbers" were ticked. Importantly, the "Results Table Parameters" should be set on: "Store results within a table named after the image", which allows to keep track of the files in batch mode. Finally, the measures were "Redirected to" the defence marker channel. After setting all the parameters, the analyse "3D Object Counter" was run. Threshold was set to 1 and

The process gives in output four different files. The "Centroid map" shows the centre of each ROI by a dot, numbered accordingly. The "Object map" is the representation of all ROIs, each of them being numbered. Our macro merge this map to both the cell wall marker channel and the nuclei channel for later identification of nuclei. The 3D Object counter also provides a .csv file called "Statistics" with all parameters measured for each nucleus. The last file provided is the "Masked image", which represent the defence marker channel masked by the threshold.

To terminate the quantification, each data set was manually curated to assign a tissue (epidermis, cortex, endodermis, stele or root cap) to each nucleus. A maximum of around 20 nuclei by tissue type and by picture were identified. "Mean Gray Values" was extracted and use for analysis. Mean nuclear intensity for each genotype, treatment, root region and tissue were calculated and colour coded using

518 the heatmaply() function in R (heatmaply library). Atlas maps were drawn according to these coloured 519 values. Fold changes for MYB51 induction were calculated and colour-coded using the same 520 procedure.

\section{$521 \mathrm{Ca}^{2+}$ imaging on roots and quantification}

522 For calcium responses analysis, UBQ10::R-GECO1 samples were mounted as follows. Seedlings, once 
Medical Adhesive Spray, Hollister). A silicon isolator (Grace Bio-Labs Press-to-seal silicon isolator, No PSA, $20 \mathrm{~mm}$ diameter, Sigma) was then quickly placed around the seedling and $600 \mu$ of sterile germination medium $\left(0.75 \mathrm{mM} \mathrm{CaCl}_{2}, 1 \mathrm{mM} \mathrm{KCl}, 0.25 \mathrm{mM} \mathrm{Ca}\left(\mathrm{NO}_{3}\right)_{2} \bullet 4 \mathrm{H}_{2} \mathrm{O}, 1 \mathrm{mM} \mathrm{MgSO} \mathrm{m}_{4} \bullet 7 \mathrm{H}_{2} 0,0.2 \mathrm{mM}\right.$ $\mathrm{KH}_{2} \mathrm{PO}_{4}, 50 \mu \mathrm{M} \mathrm{NaFe}(\mathrm{III})$ EDTA, $50 \mu \mathrm{M} \mathrm{H}_{3} \mathrm{BO}_{3}, 5 \mu \mathrm{M} \mathrm{MnCl} \mathrm{ML}_{2} \bullet 4 \mathrm{H}_{2} \mathrm{O}, 10 \mu \mathrm{MnSO}_{4} \bullet 7 \mathrm{H}_{2} \mathrm{O}, 0.5 \mu \mathrm{M} \mathrm{CuSO} \mathrm{C}_{4} \bullet 5 \mathrm{H}_{2} \mathrm{O}$, $0.1 \mu \mathrm{M} \mathrm{Na}_{2} \mathrm{MoO}_{3}, \mathrm{pH}$ adjusted to 5.6 with $\mathrm{NaOH}$ ) was dropped on the root. The drop was spread with a pipet tip to cover the whole surface delimited by the silicon isolator and the seedling let to rest for at least $20 \mathrm{~min}$. For full root imaging, tile scans combined to time laps were performed under Zeiss LSM880 confocal laser scanning microscope with $20 x$ objective as described above. As few tiles as possible were selected to limit time acquisition, no averaging was done, and pinhole was entirely open. Images were taken continuously, with an average time interval of 5 to 7 seconds. Acquisition of baseline signal was performed for $5 \mathrm{~min}$, then $7.5 \mu \mathrm{l}$ of $100 \mu \mathrm{M}$ flg22 diluted in water was added to the germination medium solution. Acquisition was continued for at least $20 \mathrm{~min}$. For tissue-specific imaging and quantification, small z-stack ( 8 slices) with $5 \mu \mathrm{m}$ intervals were taken on half a root in the elongation zone for wild-type and WER::FLS2 samples, or in the differentiated zone for CASP1::/SHR::/UBQ10::FLS2.

R-GECO1 signal was quantified for each tissue on the z-stack acquisition. ROIs delimiting a tissue type were drawn manually on the most appropriate stack (i.e. that presents a clear surface view if possible), using maximum projection of 2 stacks when necessary.

Fractional fluorescence changes $\Delta \mathrm{F} / \mathrm{F}$ were calculated for each $\mathrm{ROI}$ from background corrected intensity values as $\left(F-F_{0}\right) / F_{0}$, where $F_{0}$ is equal to the average fluorescent intensity of the baseline of the measure, on 4 min from $t=0$.

\section{Suberin staining}

To highlight suberin lamellae, seedlings were fixed and stained with the methanol-based fluorol yellow staining protocol as described in (Fujita et al., 2020). Samples were imaged using the Zeiss LSM700 as described above. 


\section{Bacterial root inoculation assays}

550 PTI assays were performed by drop inoculation on agar plates. Briefly, $2 \mu$ l of bacterial suspension (cells 551 centrifuged and resuspended in fresh LB or 50\% TSB for $\mathrm{CHAO}$ and R569, respectively) of $\mathrm{OD}_{600}=0.01$ was added to the tip of 5-day-old seedlings. Once the drop dried, seedlings were grown vertically in standard conditions for 1 to 3 days. For the fast screening of bacterial isolates, roots were observed under a Leica DM 5500B epifluorescence microscope (GFP lamp). Representative pictures of roots were imaged using confocal scanning microscopy (Leica SP8) after a short wash in deionized $\mathrm{H}_{2} \mathrm{O}$.

Root growth inhibition assays were completed on agar plates inoculated with bacteria at mentioned concentrations. Briefly, bacterial cultures were grown as previously described in 50\% TSB, then centrifuged and resuspended in fresh medium. $\mathrm{OD}_{600}$ was measured and adjusted to $100 \mathrm{x}$ the desired concentration. $500 \mu \mathrm{l}$ of concentrated bacterial inoculum was then added to $50 \mathrm{ml}$ of semi solid $1 / 2 \mathrm{MS}$ medium afore cooled down to around $30^{\circ} \mathrm{C}$. Inoculated media were gently mixed by inverting several times, then poured in square petri dishes. Five-day-old WER::FLS2 and wild-type Col-0 seedlings previously grown on mesh $(15 \mathrm{~mm} \times 100 \mathrm{~mm}$, on top of the plate), were transferred with sterile forceps on the inoculated plates. Seedlings were selected for similar root size, the ones being obviously too long or too short removed from the mesh with sterile toothpicks. After transfer, root tip locations were marked for keeping track of growth, then plants were grown in standard conditions for 6 days. One from the transfers were considered. Plates were scanned at $6 \mathrm{dpi}$ and root growth measured using Fiji plugins "Simple Neurite Tracer" (Frangi et al., 1998).

\section{Statistical analysis}

571 Statistical analyses were done using R3.6.0 or Graphpad Prism 7.0 software

572 (https://www.graphpad.com/). For multiple comparison, ANOVA followed by Tukey's Honestly

573 Significant difference (HSD) test were applied when linear model assumptions were met. On the 
contrary, Kruskal-Wallis test followed by Dunn's multiple comparison test were performed. For analysis of suberization along the roots, comparisons were performed for each zone separately, and different letters indicates significant differences for a given zone (a, b, $c$ or $a^{\prime}, b^{\prime}, c^{\prime}$ or $\left.a^{\prime \prime}, b^{\prime \prime}, c^{\prime \prime}\right)$.

\section{References}

Adams-Phillips, L., Briggs, A.G., and Bent, A.F. (2010). Disruption of poly(ADP-ribosyl)ation mechanisms alters responses of Arabidopsis to biotic stress. Plant Physiol. 152, 267-280.

Alassimone, J., Fujita, S., Doblas, V.G., Dop, M. van, Barberon, M., Kalmbach, L., Vermeer, J.E.M., RojasMurcia, N., Santuari, L., Hardtke, C.S., et al. (2016). Polarly localized kinase SGN1 is required for Casparian strip integrity and positioning. Nat. Plants 2, 1-10.

Andersen, T.G., Barberon, M., and Geldner, N. (2015). Suberization - the second life of an endodermal cell. Curr. Opin. Plant Biol. 28, 9-15.

Andersen, T.G., Naseer, S., Ursache, R., Wybouw, B., Smet, W., De Rybel, B., Vermeer, J.E.M., and Geldner, N. (2018). Diffusible repression of cytokinin signalling produces endodermal symmetry and passage cells. Nature 555, 529-533.

Arora, S.K., Ritchings, B.W., Almira, E.C., Lory, S., and Ramphal, R. (1997). A transcriptional activator, FleQ, regulates mucin adhesion and flagellar gene expression in Pseudomonas aeruginosa in a cascade manner. J. Bacteriol. 179, 5574-5581.

Bai, Y., Müller, D.B., Srinivas, G., Garrido-Oter, R., Potthoff, E., Rott, M., Dombrowski, N., Münch, P.C., Spaepen, S., Remus-Emsermann, M., et al. (2015). Functional overlap of the Arabidopsis leaf and root microbiota. Nature 528, 364-369.

Beck, M., Wyrsch, I., Strutt, J., Wimalasekera, R., Webb, A., Boller, T., and Robatzek, S. (2014). Expression patterns of FLAGELLIN SENSING 2 map to bacterial entry sites in plant shoots and roots. J. Exp. Bot. 65, 6487-6498.

Benfey, P.N., Linstead, P.J., Roberts, K., Schiefelbein, J.W., Hauser, M.T., and Aeschbacher, R.A. (1993). Root development in Arabidopsis: four mutants with dramatically altered root morphogenesis. Development 119, 57-70.

Berendsen, R.L., Pieterse, C.M.J., and Bakker, P.A.H.M. (2012). The rhizosphere microbiome and plant health. Trends Plant Sci. 17, 478-486.

Bernards, M.A. (2002). Demystifying suberin. Can. J. Bot. 80, 227-240.

Bolte, S., and Cordelières, F.P. (2006). A guided tour into subcellular colocalization analysis in light microscopy. J. Microsc. 224, 213-232.

Bulgarelli, D., Schlaeppi, K., Spaepen, S., van Themaat, E.V.L., and Schulze-Lefert, P. (2013). Structure and Functions of the Bacterial Microbiota of Plants. Annu. Rev. Plant Biol. 64, 807-838. 
Buscaill, P., Chandrasekar, B., Sanguankiattichai, N., Kourelis, J., Kaschani, F., Thomas, E.L., Morimoto, K., Kaiser, M., Preston, G.M., and Ichinose, Y. (2019). Glycosidase and glycan polymorphism control hydrolytic release of immunogenic flagellin peptides. PLANT Sci. 12.

Chezem, W.R., Memon, A., Li, F.-S., Weng, J.-K., and Clay, N.K. (2017). SG2-type R2R3-MYB transcription factor MYB15 controls defense-induced lignification and basal immunity in Arabidopsis. Plant Cell Online tpc.00954.2016.

Chinchilla, D., Zipfel, C., Robatzek, S., Kemmerling, B., Nürnberger, T., Jones, J.D.G., Felix, G., and Boller, T. (2007). A flagellin-induced complex of the receptor FLS2 and BAK1 initiates plant defence. Nature $448,497-500$.

Clough, S.J., and Bent, A.F. (1998). Floral dip: a simplified method for Agrobacterium-mediated transformation of Arabidopsis thaliana. Plant J. 16, 735-743.

Couto, D., and Zipfel, C. (2016). Regulation of pattern recognition receptor signalling in plants. Nat. Rev. Immunol. 16, 537.

De Coninck, B., Timmermans, P., Vos, C., Cammue, B.P.A., and Kazan, K. (2015). What lies beneath: belowground defense strategies in plants. Trends Plant Sci. 20, 91-101.

Doblas, V.G., Smakowska-Luzan, E., Fujita, S., Alassimone, J., Barberon, M., Madalinski, M., Belkhadir, Y., and Geldner, N. (2017). Root diffusion barrier control by a vasculature-derived peptide binding to the SGN3 receptor. Science 355, 280-284.

Dubiella, U., Seybold, H., Durian, G., Komander, E., Lassig, R., Witte, C.-P., Schulze, W.X., and Romeis, T. (2013). Calcium-dependent protein kinase/NADPH oxidase activation circuit is required for rapid defense signal propagation. Proc. Natl. Acad. Sci. 110, 8744-8749.

Faulkner, C., and Robatzek, S. (2012). Plants and pathogens: putting infection strategies and defence mechanisms on the map. Curr. Opin. Plant Biol. 15, 699-707.

Felix, G., Duran, J.D., Volko, S., and Boller, T. (1999). Plants have a sensitive perception system for the most conserved domain of bacterial flagellin. Plant J. 18, 265-276.

Fendrych, M., Van Hautegem, T., Van Durme, M., Olvera-Carrillo, Y., Huysmans, M., Karimi, M., Lippens, S., Guérin, C.J., Krebs, M., Schumacher, K., et al. (2014). Programmed cell death controlled by ANAC033/SOMBRERO determines root cap organ size in Arabidopsis. Curr. Biol. 24, 931-940.

Frangi, A.F., Niessen, W.J., Vincken, K.L., and Viergever, M.A. (1998). Multiscale vessel enhancement filtering. In Medical Image Computing and Computer-Assisted Intervention - MICCAI'98, W.M. Wells, A. Colchester, and S. Delp, eds. (Berlin, Heidelberg: Springer), pp. 130-137.

Fujita, S., De Bellis, D., Edel, K.H., Köster, P., Andersen, T.G., Schmid-Siegert, E., Dénervaud Tendon, V., Pfister, A., Marhavý, P., Ursache, R., et al. (2020). SCHENGEN receptor module drives localized ROS production and lignification in plant roots. EMBO J. $n / a$, e103894.

Garrido-Oter, R., Nakano, R.T., Dombrowski, N., Ma, K.-W., McHardy, A.C., and Schulze-Lefert, P. (2018). Modular traits of the rhizobiales root microbiota and their evolutionary relationship with symbiotic rhizobia. Cell Host Microbe 24, 155-167.e5.

Gilroy, S., Suzuki, N., Miller, G., Choi, W.-G., Toyota, M., Devireddy, A.R., and Mittler, R. (2014). A tidal wave of signals: calcium and ROS at the forefront of rapid systemic signaling. Trends Plant Sci. 19, 623630. 
Gilroy, S., Białasek, M., Suzuki, N., Górecka, M., Devireddy, A., Karpinski, S., and Mittler, R. (2016). ROS, calcium and electric signals: key mediators of rapid systemic signaling in plants. Plant Physiol. pp.00434.2016.

Gómez-Gómez, L., and Boller, T. (2000). FLS2: An LRR receptor-like kinase involved in the perception of the bacterial elicitor flagellin in Arabidopsis. Mol. Cell 5, 1003-1011.

Gómez-Gómez, L., Felix, G., and Boller, T. (1999). A single locus determines sensitivity to bacterial flagellin in Arabidopsis thaliana. Plant J. 18, 277-284.

Gul-Mohammed, J., Arganda-Carreras, I., Andrey, P., Galy, V., and Boudier, T. (2014). A generic classification-based method for segmentation of nuclei in 3D images of early embryos. BMC Bioinformatics 15, 9.

Hara, M., Umetsu, N., Miyamoto, C., and Tamari, K. (1973). Inhibition of the biosynthesis of plant cell wall materials, especially cellulose biosynthesis, by coumarin. Plant Cell Physiol. 14, 11-28.

Helariutta, Y., Fukaki, H., Wysocka-Diller, J., Nakajima, K., Jung, J., Sena, G., Hauser, M.-T., and Benfey, P.N. (2000). The SHORT-ROOT gene controls radial patterning of the Arabidopsis root through radial signaling. Cell 101, 555-567.

Hijwegen, T. (1963). Lignification, a possible mechanism of active resistance against pathogens. Neth. J. Plant Pathol. 69, 314-317.

Jeworutzki, E., Roelfsema, M.R.G., Anschütz, U., Krol, E., Elzenga, J.T.M., Felix, G., Boller, T., Hedrich, R., and Becker, D. (2010). Early signaling through the Arabidopsis pattern recognition receptors FLS2 and EFR involves Ca2+-associated opening of plasma membrane anion channels. Plant J. 62, 367-378.

Kamula, S.A., Peterson, C.A., and Mayfield, C.I. (1994). Impact of the exodermis on infection of roots by Fusarium culmorum. Plant Soil 167, 121-126.

Keinath, N.F., Waadt, R., Brugman, R., Schroeder, J.I., Grossmann, G., Schumacher, K., and Krebs, M. (2015). Live cell imaging with R-GECO1 sheds light on flg22- and chitin-induced transient [Ca2+]cyt patterns in Arabidopsis. Mol. Plant 8, 1188-1200.

Kumpf, R.P., and Nowack, M.K. (2015). The root cap: a short story of life and death. J. Exp. Bot. 66, 5651-5662.

Kupferschmied, P. (2015). Molecular basis and regulation of insect pathogenicity in plant-beneficial pseudomonads. Université de Lausanne, Faculté de biologie et médecine.

Kupferschmied, P., Péchy-Tarr, M., Imperiali, N., Maurhofer, M., and Keel, C. (2014). Domain shuffling in a sensor protein contributed to the evolution of insect pathogenicity in plant-beneficial Pseudomonas protegens. PLOS Pathog. 10, e1003964.

Kurihara, D., Mizuta, Y., Sato, Y., and Higashiyama, T. (2015). ClearSee: a rapid optical clearing reagent for whole-plant fluorescence imaging. Development 142, 4168-4179.

Lange, B.M., Lapierre, C., and Jr, H.S. (1995). Elicitor-induced spruce stress lignin (structural similarity to early developmental lignins). Plant Physiol. 108, 1277-1287.

Lee, M.M., and Schiefelbein, J. (1999). WEREWOLF, a MYB-related protein in Arabidopsis, is a positiondependent regulator of epidermal cell patterning. Cell 99, 473-483. 
Lee, M.-H., Jeon, H.S., Kim, S.H., Chung, J.H., Roppolo, D., Lee, H.-J., Cho, H.J., Tobimatsu, Y., Ralph, J., and Park, O.K. (2019). Lignin-based barrier restricts pathogens to the infection site and confers resistance in plants. EMBO J. 38, e101948.

Li, B., Meng, X., Shan, L., and He, P. (2016). Transcriptional Regulation of Pattern-Triggered Immunity in Plants. Cell Host Microbe 19, 641-650.

Mandal, S., and Mitra, A. (2007). Reinforcement of cell wall in roots of Lycopersicon esculentum through induction of phenolic compounds and lignin by elicitors. Physiol. Mol. Plant Pathol. 71, 201209.

Marhavý, P., Kurenda, A., Siddique, S., Dénervaud Tendon, V., Zhou, F., Holbein, J., Hasan, M.S., Grundler, F.M., Farmer, E.E., and Geldner, N. (2019). Single-cell damage elicits regional, nematoderestricting ethylene responses in roots. EMBO J. 38, e100972.

Marquès-Bueno, M.M., Morao, A.K., Cayrel, A., Platre, M.P., Barberon, M., Caillieux, E., Colot, V., Jaillais, Y., Roudier, F., and Vert, G. (2015). A versatile Multisite Gateway-compatible promoter and transgenic line collection for cell type-specific functional genomics in Arabidopsis. Plant J. n/a-n/a.

Martínez-García, E., and Lorenzo, V. de (2011). Engineering multiple genomic deletions in Gramnegative bacteria: analysis of the multi-resistant antibiotic profile of Pseudomonas putida KT2440. Environ. Microbiol. 13, 2702-2716.

Messner, B., and Boll, M. (1993). Elicitor-mediated induction of enzymes of lignin biosynthesis and formation of lignin-like material in a cell suspension culture of spruce (Picea abies). Plant Cell Tissue Organ Cult. 34, 261-269.

Millet, Y.A., Danna, C.H., Clay, N.K., Songnuan, W., Simon, M.D., Werck-Reichhart, D., and Ausubel, F.M. (2010). Innate Immune Responses Activated in Arabidopsis Roots by Microbe-Associated Molecular Patterns. Plant Cell 22, 973-990.

Nicholson, R.L., and Hammerschmidt, R. (1992). Phenolic Compounds and Their Role in Disease Resistance. Annu. Rev. Phytopathol. 30, 369-389.

Ollion, J., Cochennec, J., Loll, F., Escudé, C., and Boudier, T. (2013). TANGO: a generic tool for highthroughput 3D image analysis for studying nuclear organization. Bioinformatics 29, 1840-1841.

Pel, M.J.C., and Pieterse, C.M.J. (2013). Microbial recognition and evasion of host immunity. J. Exp. Bot. 64, 1237-1248.

Pfister, A., Barberon, M., Alassimone, J., Kalmbach, L., Lee, Y., Vermeer, J.E., Yamazaki, M., Li, G., Maurel, C., Takano, J., et al. (2014). A receptor-like kinase mutant with absent endodermal diffusion barrier displays selective nutrient homeostasis defects. ELife 3, e03115.

Poncini, L., Wyrsch, I., Tendon, V.D., Vorley, T., Boller, T., Geldner, N., Métraux, J.-P., and Lehmann, S. (2017). In roots of Arabidopsis thaliana, the damage-associated molecular pattern AtPep1 is a stronger elicitor of immune signalling than flg22 or the chitin heptamer. PLOS ONE 12, e0185808.

Ranathunge, K., Thomas, R.H., Fang, X., Peterson, C.A., Gijzen, M., and Bernards, M.A. (2008). Soybean Root Suberin and Partial Resistance to Root Rot Caused by Phytophthora sojae. Phytopathology 98, 1179-1189. 

K., Esteban, E., Pasha, A., Kogel, K.-H., et al. (2020). Regulation of Cell Type-Specific Immunity Networks in Arabidopsis Roots. Plant Cell.

Robatzek, S., Chinchilla, D., and Boller, T. (2006). Ligand-induced endocytosis of the pattern recognition receptor FLS2 in Arabidopsis. Genes Dev. 20, 537-542.

Robertsen, B. (1986). Elicitors of the production of lignin-like compounds in cucumber hypocotyls. Physiol. Mol. Plant Pathol. 28, 137-148.

Schneider, C.A., Rasband, W.S., and Eliceiri, K.W. (2012). NIH Image to ImageJ: 25 years of image analysis. Nat. Methods 9, 671-675.

Seybold, H., Trempel, F., Ranf, S., Scheel, D., Romeis, T., and Lee, J. (2014). Ca2+ signalling in plant immune response: from pattern recognition receptors to $\mathrm{Ca}^{2+}$ decoding mechanisms - Seybold - 2014 - - Wiley Online Library. New Phytol. Prasad, K., et al. (2016). MultiSite Gateway-Compatible Cell Type-Specific Gene-Inducible System for Plants. Plant Physiol. 170, 627-641.

Smit, F., and Dubery, I.A. (1997). Cell wall reinforcement in cotton hypocotyls in response to a Verticillium dahliae elicitor. Phytochemistry 44, 811-815.

740

741

742

743

Stanley, C.E., Shrivastava, J., Brugman, R., Heinzelmann, E., Swaay, D. van, and Grossmann, G. (2018). Dual-flow-RootChip reveals local adaptations of roots towards environmental asymmetry at the physiological and genetic levels. New Phytol. 217, 1357-1369.

Stringlis, I.A., de Jonge, R., and Pieterse, C.M.J. (2019). The Age of Coumarins in Plant-Microbe Interactions. Plant Cell Physiol.

Stutz, E.W., Défago, G., and Kern, H. (1986). Naturally occurring fluorescent pseudomonads involved in suppression of black root rot of tobacco. Phytopathology 76, 181-185.

Tang, D., Wang, G., and Zhou, J.-M. (2017). Receptor Kinases in Plant-Pathogen Interactions: More Than Pattern Recognition. Plant Cell 29, 618-637.

Thomas, R., Fang, X., Ranathunge, K., Anderson, T.R., Peterson, C.A., and Bernards, M.A. (2007). Soybean Root Suberin: Anatomical Distribution, Chemical Composition, and Relationship to Partial Resistance to Phytophthora sojae. Plant Physiol. 144, 299-311.

Thor, K., and Peiter, E. (2014). Cytosolic calcium signals elicited by the pathogen-associated molecular pattern flg22 in stomatal guard cells are of an oscillatory nature. New Phytol. 204, 873-881.

Tognolli, M., Penel, C., Greppin, H., and Simon, P. (2002). Analysis and expression of the class III peroxidase large gene family in Arabidopsis thaliana. Gene 288, 129-138.

Ursache, R., Andersen, T.G., Marhavý, P., and Geldner, N. (2018). A protocol for combining fluorescent proteins with histological stains for diverse cell wall components. Plant J. 93, 399-412.

Vance, C.P., Kirk, T.K., and Sherwood, R.T. (1980). Lignification as a Mechanism of Disease Resistance. Annu. Rev. Phytopathol. 18, 259-288. 
Vermeer, J.E.M., Wangenheim, D. von, Barberon, M., Lee, Y., Stelzer, E.H.K., Maizel, A., and Geldner, N. (2014). A Spatial Accommodation by Neighboring Cells Is Required for Organ Initiation in Arabidopsis. Science 343, 178-183.

Wyrsch, I., Domínguez-Ferreras, A., Geldner, N., and Boller, T. (2015). Tissue-specific FLAGELLINSENSING 2 (FLS2) expression in roots restores immune responses in Arabidopsis fls2 mutants. New Phytol. 206, 774-784.

Yamaguchi, S., Fujita, H., Sugata, K., Taira, T., and lino, T. (1984). Genetic Analysis of H2, the Structural Gene for Phase-2 Flagellin in Salmonella. Microbiology, 130, 255-265.

Yu, K., Pieterse, C.M.J., Bakker, P.A.H.M., and Berendsen, R.L. (2019a). Beneficial microbes going underground of root immunity. Plant Cell Environ. 42, 2860-2870.

Yu, K., Liu, Y., Tichelaar, R., Savant, N., Lagendijk, E., Kuijk, S.J.L. van, Stringlis, I.A., Dijken, A.J.H. van, Pieterse, C.M.J., Bakker, P.A.H.M., et al. (2019b). Rhizosphere-Associated Pseudomonas Suppress Local Root Immune Responses by Gluconic Acid-Mediated Lowering of Environmental pH. Curr. Biol. 0 . Co-incidence of Damage and Microbial Patterns Controls Localized Immune Responses in Roots. Cell 775 180, 440-453.e18.

Zipfel, C. (2008). Pattern-recognition receptors in plant innate immunity. Curr. Opin. Immunol. 20, 1016. 
782

783

Figure 1: Tissue-specific promoters drive FLS2 receptor expression ectopically

(A) Tile scan of $f / s 2$ roots complemented with GFP-tagged FLS2 receptor under epidermal (WER::), endodermal (CASP1::), central cylinder (SHR::) and ubiquitous (UBQ10::) promoters. For comparison, endogenous FLS2 expression is shown in FLS2::FLS2-GFP Ws-0 lines. Root shape is highlighted with PI staining cell wall $(\mathrm{PI}, \mathrm{red})$. Scale bar, $100 \mu \mathrm{m}$. Developmental regions of the roots are labelled: differentiated zone (DZ), elongation zone (EZ), meristematic zone (MZ). (B) Close up view of FLS2-GFP expression at selected regions of the complemented lines. FLS2 driven by its endogenous promoter is expressed in all tissues in the differentiated zone (DZ). Note that in contrast to previous report, low FLS2 expression is observed in epidermis and cortex (white arrow). In the meristem (MZ), WER promoter expresses FLS2 specifically in epidermis (ep) and root cap (rc), SHR promoter in the stele (st) and endodermis (en). In the differentiated zone (DZ), FLS2 is expressed in all tissues under UBQ10 promoter, but is restricted to endodermis with CASP1 promoter. FLS2-GFP (BlueGreen) is co-visualized with PI-stained cell wall (red). Separated and overlaid channels (right column) are presented. Scale bar, $25 \mu$ m. ep, epidermis; c, cortex; en, endodermis; st, stele; rc, root cap cells.

\section{Figure 2: PER5 marker gene is induced cell-autonomously by flg22 treatment}

(A) Overview of PER5::NLS-3mVenus marker response to flg22 in different FLS2 recombinant lines. Tile scan images of $1 \mu \mathrm{M}$ flg22 treated plants versus mock. Pictures were taken with similar settings. Settings were always identical between mock and corresponding flg22 treatment. Region of responsiveness is modified by the different expression patterns of $F L S 2$. Brackets indicate responsive regions. For SHR, close-up view was generated with increased brightness to highlight stellar signal (white arrow). Scale bar, $100 \mu \mathrm{m}$. (B) Maximal projection of transverse sections views of PER5 expression pattern in UBQ10:: and WER::FLS2-GFP fls2 compared to WT shown for meristematic zone (MZ), elongation zone (EZ) and differentiated zone (DZ, 30 cells after start of elongation). Seedlings were treated for $24 \mathrm{~h}$ with $1 \mu \mathrm{M}$ flg22. Note the refractory region in the central cylinder in UBQ10::FLS2 
806

807

808

809

810

811

812

813

814

815

816

817

818

819

820

821

822

823

824

825

826

827

828

(white arrows). Nuclear localized mVenus signal (yellow) was co-displayed with propidium iodide cell wall marker ( $\mathrm{PI}$, purple). Images were taken with similar settings, but corresponding mock and flg22 treatment pictures for each zone separately always have identical parameters. Note that epidermal signal in flg22-treated wild-type seedlings is faint (EZ, black arrow), due to settings chosen to avoid saturation of signal in the transgenic lines. Compare to Fig.2C, WT. Scale bar, $25 \mu \mathrm{m}$. (C) Maximal projection of transverse section views of PER5::NLS-3mVenus expression pattern in CASP1:: and SHR::FLS2-GFP fls2 as well as WT and fls2 control. White arrows point at ectopic response in the endodermis. Images were acquired as in Fig.2B., with similar settings between genotypes, but with identical parameter for corresponding mock and flg22 treatment. Pictures were acquired with increased gain compared to Fig.2B due to lower average signal intensity. Scale bar, $25 \mu \mathrm{m}$. (D) Quantitative map of PER5::NLS-3mVenus responses inferred from tissue specific quantification after 24h treatment with $1 \mu \mathrm{M}$ flg22. Nuclear signals were quantified in ROI delimited with UBQ10::NLSmTurquoises2 for all tissue-specific promoter lines, while wild-type (WT) signal was quantified with UBQ10::NLS-tdTomato marker. Mean intensity is therefore comparable between prom::FLS2-GFP fls2 lines, but not to wild-type.

\section{Figure 3: $\mathrm{Ca}^{2+}$ waves are non-cell autonomous responses}

(A) $\mathrm{Ca}^{2+}$-dependent signal in the prom::FLS2-GFP fls2 lines in response to $1.25 \mu \mathrm{M}$ flg22. Time series of UBQ10::R-GECO1 fluorescence: pictures are longitudinal middle sections of roots at the elongation zone (EZ) or differentiated zone (DZ). Time 0:00 corresponds to the start of flg22 treatment. White arrows point at tissues showing a strong increase in $\mathrm{Ca}^{2+}$ content. Scale bar, $25 \mu \mathrm{m}$. (B) Normalized RGECO1 fluorescence intensity ( $\Delta F / F)$ measured in tissue-specific ROIs. Values present the dynamics of $\mathrm{Ca}^{2+}$ cytosolic concentration in response to flg22 in the root shown in (A) for each tissue type. Black arrows point at the maximum intensity of the trace. Grey background corresponds to flg22 treatment. 
832 (A) Flg22 treatment increases root growth inhibition in WER::FLS2 hypersensitive line. Root length quantification of prom::FLS2-GFP fls2 lines transferred on $1 \mu \mathrm{M}$ flg22 for $6 \mathrm{dpi}$. Boxplot centre represents the median ( $n=23$ to 28 roots). Different letters indicate statistically significant difference between means by Kruskal-Wallis test and Dunn's multiple comparison. (B) Representative pictures of seedlings transferred for 6 days on $1 \mu \mathrm{M}$ flg22. Scale bar, $1 \mathrm{~cm}$. (C) Flg22 induces strongly PER5::NLS$3 m$ Venus in the epidermis of WER::FLS2-GFP fIs2 hypersensitive line. On the right, maximum projection of $z$-stacks taken in root tips of plants treated for $24 \mathrm{~h}$ with $1 \mu \mathrm{M}$ flg22 or mock. Schematic represents the depth of the z-stack. Pictures were taken with identical settings. Scale bar, $25 \mu \mathrm{m}$. (D) WER::FLS2GFP fls2 hypersensitivity is specific to flg22. WER::FLS2-GFP fls2 and wild-type plants were treated for $24 \mathrm{~h}$ with either $1 \mu \mathrm{M}$ elf18, $2 \mu \mathrm{g} / \mathrm{ml}$ chitin, $1 \mu \mathrm{M} 3-\mathrm{OH}-\mathrm{C} 10: 0$ or $1 \mu \mathrm{M}$ AtPep1. Maximum projection of zstacks taken in root tips. PER5 induction is highlighted with mVenus (Fire LUT). Parameters were identical for mock and treatment. Scale bar, $25 \mu \mathrm{m}$. (E) Treatment of WER::FLS2-GFP fls2 for 2 days with $1 \mu \mathrm{M}$ flg22 induces meristem swelling and lignin deposition. Upper panel shows median projection of calcofluor white stained cell wall in the transition zone of the root tip (blue). Note bulky cells of the epidermis (white arrowhead). Lower panel presents maximum projection of lignin deposition stained with basic fuchsin (red). Lignin accumulates between cells only in WER::FLS2-GFP fls2 after flg22 treatment. Scale bar, $25 \mu \mathrm{m}$. (F) Cross-section of pictures in (E). Cell wall stained with calcofluor white (blue) is co-visualized with lignin stained with basic fuchsin (red). Flg22 treatment induces massive sometimes missing. Scale bar, $25 \mu \mathrm{m}$. (G) Epidermal view of plasma membrane visualized by the construct UBQ10::mScarlet-SYP122 in WER::FLS2-GFP fls2. Cell division is disorganized after $1 \mu \mathrm{M}$ flg22 treatment. Scale bar, $25 \mu \mathrm{m}$. (H) Inhibition of monolignol synthesis does not rescue meristem flg22driven increased root growth inhibition of WER::FLS2-GFP fls2. Root growth measured after overnight 

Boxplot centre represents the median $(16<=n<=27)$. Different letters indicate statistically significant difference $(p<0.05)$ between means by Kruskal-Wallis test and Dunn's multiple comparison. (I) Flg22 induces meristem swelling despite inhibition of monolignol by PA treatment. Pictures taken from samples quantified in $(\mathrm{H})$. Upper panel shows median projection of calcofluor white stained cell wall in the transition zone of root tip (blue). Lower panel presents maximum projection of lignin deposition stained with basic fuchsin (red). White arrowheads points at examples of bulky cells. Scale bar, $25 \mu \mathrm{m}$.

(A) Quantification of suberized zone length in seedlings treated for 1 day with $1 \mu \mathrm{M}$ flg22 (18<=n <= significant differences amongst lines for the specified zone $(p<0.05)$. Multiple comparison was performed using ANOVA and Tukey's tests for the suberized zone, whereas Kruskal-Wallis and Dunn's suberin lamellae deposition in CASP1:: and UBQ10::FLS2-GFP fIs2 lines compared to wild-type after $1 \mu \mathrm{M}$ flg22 treatment vs mock. Suberin was stained with fluorol yellow. White arrowheads start of 872 patchy zone; yellow arrowheads, start of fully suberized zones. Scale bar, $1 \mathrm{~mm}$.

\section{Figure 6: WER::FLS2 line detects endogenous bacterial flg22}

874 (A) CHAO bacteria trigger a strong induction of PER5::NLS-3mVenus marker (Fire LUT) on WER::FLS2GFP fls2. Mutants $\triangle$ fliC and $\Delta$ fleQ defective for flagellum lose their ability to induce detectable MTI. $\Delta$ fliC mutant was confirmed by motility assay (see Fig.S7D). Maximum projection of z-stacks imaging meristematic (MZ) and elongation (EZ) zones treated with drop inoculation of bacterial solution of a concentration of $\mathrm{OD}_{600}=0.01$ or mock, respectively. Images were acquired at $1 \mathrm{dpi}$. Acquisition done with identical settings. Scale bar, $25 \mu \mathrm{m}$. (B) CHAO do not induce consistently increased root growth 
881 at $\mathrm{OD}_{600}=10^{-3}$. Different letters indicate statistically significant differences $(p<0.05)$. Multiple

882 comparison was performed using ANOVA and Tukey's test. (C) Proportion of natural isolates from At-

883 SPHERE culture collection triggering stronger PER5::NLS-3mVenus induction and increased root growth

884 inhibition (RGI) on WER::FLS2-GFP fls2 compared to wild-type seedlings (yes), or not (no). Bacteria

885 classified in "variable" presented contradictory results between replicates. Bacteria flg22 sequence

886 was predicted to be recognized by FLS2 (flg22 predicted) or not (flg22 not predicted). Numbers of

887 bacterial isolates in each category are indicated in colour. Grey surfaces indicate identical bacteria

888 strains. (D) Pseudomonas isolate R569 from At-SPHERE culture collection triggers strong PER5::NLS-

$8893 m$ Venus (Fire LUT ) induction on WER::FLS2-GFP fls2. Seedlings were imaged after one-day treatment

890 with $\mathrm{OD}_{600}=0.01$. Maximum projection of z-stacks at meristematic zone (MZ) and elongation zone (EZ).

891 Scale bar, $25 \mu \mathrm{m}$. (E) Isolate R569 induces a robust increased root growth inhibition on WER::FLS2-GFP

892 fls2 compared to wild-type plants. High concentration of bacteria $\left(\mathrm{OD}_{600}=0.1\right)$ is deleterious to both

893 genotypes. Root growth was quantified at 6 dpi on plate inoculated with bacteria at $O D_{600}=10^{-1}$ to $10^{-}$

$894{ }^{4}$. Different letters indicate statistically significant differences $(p<0.05)$. Multiple comparison was 895 performed using Kruskal-Wallis and Dunn's test. 


\section{Supplemental Information Titles and Legends}

897

898

899

900

901

902

903

904

905

906

\section{Supplemental Figure S1: Expression pattern of prom::FLS2 complementing fls2.}

(A) WER::FLS2-GFP expression. WER promoter expressed principally FLS2 in epidermal cells, but some weak signal can be observed in cortex (black arrowheads). Picture of wild-type plants taken with identical setting (EZ-wt) is showed for comparison (cortical cell, white arrowhead). (B) CASP1::FLS2GFP is expressed exclusively in endodermal cell line in early and later differentiated zones (15 cells respectively 30 cells after onset of elongation). (C) UBQ10::FLS2-GFP is expressed in all tissue types in every region of the root. (D) SHR::FLS2-GFP is expressed strongly in the stele of the meristem then decreases in intensity in later regions. Some weak signal can be detected in endodermal cells (black arrowheads). Picture of wild-type plants taken with identical setting (DZ-wt) is shown for comparison (endodermal cells, white arrowheads). Close-up view of dashed squared box is found in (a). FLS2-GFP (visualized by ICA and Thermal LUTs) is merged with cell wall stained by PI (white). White arrowheads point at endodermal cells expressing weakly FLS2. (E) $R C H 1$ promoter expresses $F L S 2$ in the meristem, PRP3:: in the root hair cells and GRP:: in the pericycle cells. FLS2-GFP (green) is co-visualized with PIstained cell wall (magenta). (F) Flg22 treatment increases root growth inhibition in WER:: and RCH1::FLS2-GFP fls2 hypersensitive line only. Root length quantification of prom::FLS2-GFP fls2 lines treated with $1 \mu \mathrm{M}$ flg22 for 2 days. Boxplot centre represents the median $(5<=\mathrm{n}<=14)$. Different letters indicate statistically significant difference between means by 2-ways ANOVA and Tukey's multiple comparison. (G) Maximal projection PER5::NLS-3mVenus marker (Fire LUT) in RCH1::FLS2-GFP fls2 compared to WT shown for MZ. Seedlings were treated for $24 \mathrm{~h}$ with $1 \mu \mathrm{M}$ flg22. Images were taken with identical settings. White arrow, epidermal signal. (H) Maximal projection PER5::NLS-3mVenus marker (Fire LUT) in PRP3::FLS2-GFP fIs2 compared to WT shown for MZ. Seedlings were treated for 24h with $1 \mu \mathrm{M}$ flg22. Images were taken with identical settings. (I) Maximal projection PER5::NLS3mVenus marker (Fire LUT) in GRP::FLS2-GFP fls2 compared to WT shown for the DZ. Seedlings were treated for $24 \mathrm{~h}$ with $1 \mu \mathrm{M}$ flg22. Images were taken with identical settings. (J) LBD16 promoter expresses FLS2-GFP in all tissues in the differentiated zone (DZ). Note that in contrast to previous 
922

923

report, FLS2 is present in epidermis, cortex and endodermis (white arrows) in addition to the stele. Meristematic zone (MZ), elongation zone (EZ), differentiation zone (DZ). Scale bar, $25 \mu \mathrm{M}$.

\section{Supplemental Figure S2: Quantification procedure}

(A) Z-stack images with 3 channels (red: cell wall, blue: UBQ10::NLS-mTurquoise/tdTomato, yellow: PER5/MYB51::NLS-3mVenus) were taken from 4 different regions of the root (meristematic zone, elongation zone, 12 cells and 30 cells after onset of elongation), for 3-6 seedlings by treatment by genotype. (B) Each Z-stacks are resliced to get cross-section view. The three channels are separated (C) and a Gaussian blur filter is applied on the cell wall and the MTI marker channel (D). Blurred cell wall channel is then subtracted from blurred nuclear marker channel to remove non-nuclear background (E). The obtained cleaned nuclear channel is then converted to 8-bit and a 3D iterative thresholding is performed to delimit ROI for each nuclei (F). The 3D object counter plugin is then used to measure the mean signal intensity of each nuclei delimited by the obtained ROIs in the MTI marker channel. The plugin gives as output a .csv file with the measured values, a masked image of the PTI marker channel and an object map, delimiting the identified nuclei (G). The object map is then coupled to the original cell wall marker to define the tissue origin of each nuclei $(\mathbf{H})$. Each map was then reviewed manually to assign 20 nuclei for each cell type and to complete .csv files (I). Average of the mean signal intensity of each nuclear tissue-specific signal were calculated, transformed into $\log _{10}$ and colour coded using the heatmaply() function in R (J). Boxplots were generated to represent signal variability (K).

\section{Supplemental Figure S3: MYB51 marker is induced non cell-autonomously by flg22 treatment}

(A) Overview of MYB51::NLS-3mVenus response to $1 \mu \mathrm{M}$ flg22 after 1 day in different prom::FLS2-GFP fls2 lines. MYB51 zone of responsiveness follows FLS2 expression pattern. Tile scan images were taken with similar settings. Settings are always identical between mock and corresponding flg22 treatment.

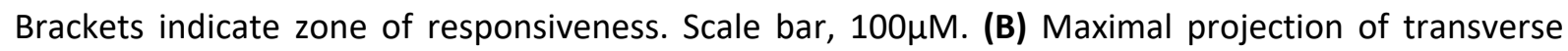
sections views of MYB51 expression pattern in UBQ10:: and WER::FLS2-GFP fls2 compared to WT 
shown for meristematic zone (MZ), elongation zone (EZ) and differentiated zone (DZ, 30 cells after start of elongation). Seedlings were treated for $24 \mathrm{~h}$ with $1 \mu \mathrm{M}$ flg22. Nuclear localized mVenus signal (yellow) was co-displayed with propidium iodide cell wall marker (PI, purple). Images were taken with similar settings, while corresponding mock and flg22 treatment pictures for each zone separately have identical parameters. Pictures were acquired with low gain compare to Fig.S2C due to strong average intensity of UBQ10:: and WER::FLS2-GFP fls2 responses, explaining the faint signal in WT (white arrowheads). Scale bar, $25 \mu \mathrm{m}$. (C) Maximal projection of transverse sections views of MYB51::NLS3mVenus expression in CASP1:: and SHR::FLS2 as well as WT and fls2. MYB51 expression pattern stay conserved (epidermis-cortex-stele), but intensity is increased in neighbourhood of cells expressing FLS2, such as in cortex in CASP1::FLS2-GFP fIs2 or stele in SHR::FLS2-GFP fls2 (white arrowheads). Imaged were acquired as Fig.S2B., with similar settings between genotypes, while corresponding mock and flg22 treatment pictures have identical parameters. Due to lower average signal intensity, pictures the constitutive signal present in untreated seedlings.

\section{Supplemental Figure S4: PER5 and MYB51 tissue-specific quantification values}

Boxplots for mean intensity of PER5::NLS-3mVenus (A-F) and MYB51::NLS-3mVenus $\left(\mathbf{A}^{\prime}-\mathbf{F}^{\prime}\right)$ marker 


\section{Supplemental Figure S5: Tissue-specific quantification of MYB51 fold change}

$973 \log _{2}$ transformed fold change of intensity of MYB51::NLS-3mVenus in WT, fls2 and the different prom::FLS2-GFP fls2 lines. Pattern of induction of MYB51 changed between the different lines but increased signal is not restricted to tissue expressing FLS2 (stars). Note that MYB51 can be induced in the stellar meristem in UBQ10::FLS2 but not in SHR::FLS2 (!). MZ, meristematic zone; EZ, elongation zone; $15 c, 15$ cells after onset of elongation; 30c, 30 cells after onset of elongation; rc, root cap; epi, epidermis; cor, cortex; endo, endodermis; ste, stele.

(A) Maximum projection showing lignin deposition stained by basic fuchsin in the meristematic zone MZ and DZ. In contrast, CASP1:: and SHR::FLS2-GFP fls2 lignified heavily the DZ only. Black arrowheads, basic fuchsin (red). WER::FLS2-GFP expression drives lignin deposition between cortex and epidermal cells in DZ, and between epidermal cells and root cap in MZ. This pattern is also observed in UBQ10::FLS2, but extends to cortex and endodermis in DZ. Both CAPS1:: and SHR:: deposit lignin ectopically between cortex and endodermal cells after flg22 treatment. White arrowheads, ectopic

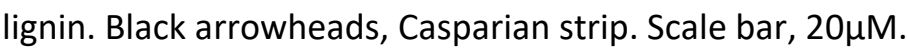


997 wild-type (WT) and strongly on WER::FLS2-GFP fls2 in the same extent than commercial flg22 for

$998 P$. aeruginosa. Seedlings were transferred for 7 days on plates containing 1 uM flg22, flg22 $2_{\text {R569 }}$ or mock.

999 Boxplot centre represents the median. Different letters indicate statistically significant difference

$1000(p<0.05)$ between means by 2-ways ANOVA and Tukey's multiple comparison tests. (C) Bacterial isolate

1001 R569 induces stronger root growth inhibition on wild-type seedlings (WT) than on WER::FLS2-GFP fls2.

1002 Replicate carried out in Cologne with different growth conditions (see material and methods). Five-

1003 days old seedlings were transferred for 11 days on plate containing bacteria at a concentration of

$1004 \mathrm{OD}_{600}=0.01$. Boxplot centre represents the median. Different letters indicate statistically significant

1005 difference $(p<0.05)$ between means by ANOVA and Tukey's multiple comparison tests. (D) Motility 1006 assay for $\mathrm{CHAO}$ and its $\triangle$ fliC mutant. 
bioRxiv preprint doi: https://doi.org/10.1101/2020.08.03.233817; this version posted August 3, 2020. The copyright holder for this preprint (which was not certified by peer review) is the author/funder. All rights reserved. No reuse allowed without permission.

\section{Supplemental Tables}


Supplemental Table S1: Summary of bacteria screen for PTI assay and RGI assay

\begin{tabular}{|c|c|c|c|c|c|c|c|c|}
\hline $\begin{array}{l}\text { Number } \\
\text { At- SPHERE }\end{array}$ & Phylum & Class & Order & Family & Genus & $\begin{array}{l}\text { flg22 } \\
\text { predicted }^{3}\end{array}$ & $\begin{array}{l}\text { MTI } \\
\text { WER>WT }\end{array}$ & $\begin{array}{l}\text { RGI } \\
\text { WER }>W T\end{array}$ \\
\hline Root 1464 & Actinobacteria & Actinobacteria & Actinomycetales & Microbacteriaceae & & no & no & no \\
\hline Root 227 & Actinobacteria & Actinobacteria & Actinomycetales & Microbacteriaceae & & no & no/yes & no \\
\hline Root 935 & Bacteroidetes & Flavobacteriia & Flavobacteriales & Flavobacteriaceae & Flavobacterium & no & no & no \\
\hline Root 444D2 & Firmicutes & Bacilli & Bacillales & Paenibacillaceae & Paenibacillus & no & yes & no \\
\hline Root 342 & Proteobacteria & Alphaproteobacteria & Caulobacterales & Caulobacteraceae & Caulobacter & no & $-a$ & no \\
\hline Root 700 & Proteobacteria & Alphaproteobacteria & Caulobacterales & Caulobacteraceae & & no & - & no \\
\hline Root 105 & Proteobacteria & Alphaproteobacteria & Rhizobiales & Hyphomicrobiaceae & & no & - & no \\
\hline Root 1471 & Proteobacteria & Alphaproteobacteria & Rhizobiales & Phyllobacteriaceae & & no & $-a$ & no \\
\hline Root 482 & Proteobacteria & Alphaproteobacteria & Rhizobiales & Rhizobiaceae & Rhizobium & no & no & no \\
\hline Root 954 & Proteobacteria & Alphaproteobacteria & Rhizobiales & Rhizobiaceae & Rhizobium & no & no & no \\
\hline Root 142 & Proteobacteria & Alphaproteobacteria & Rhizobiales & Rhizobiaceae & Sinorhizobium & no & no & no \\
\hline Root 50 & Proteobacteria & Alphaproteobacteria & Sphingomonadales & Sphingomonadaceae & Sphingomonas & yes & no & no \\
\hline Root 1294 & Proteobacteria & Alphaproteobacteria & Sphingomonadales & Sphingomonadaceae & Sphingomonas & yes & no & no \\
\hline Root 710 & Proteobacteria & Alphaproteobacteria & Sphingomonadales & Sphingomonadaceae & Sphingomonas & yes & no & no \\
\hline Root 241 & Proteobacteria & Alphaproteobacteria & Sphingomonadales & Sphingomonadaceae & Sphingomonas & yes & no/yes ${ }^{b}$ & no \\
\hline Root 1497 & Proteobacteria & Alphaproteobacteria & Sphingomonadales & Sphingomonadaceae & Sphingopyxis & yes & no & no \\
\hline Root 214 & Proteobacteria & Alphaproteobacteria & Sphingomonadales & Sphingomonadaceae & Sphingopyxis & yes & yes & no/yes \\
\hline Root 154 & Proteobacteria & Alphaproteobacteria & Sphingomonadales & Sphingomonadaceae & & yes & yes & no/yes \\
\hline Root 83 & Proteobacteria & Betaproteobacteria & Burkholderiales & Alcaligenaceae & Achromobacter & yes & no & no \\
\hline Root 170 & Proteobacteria & Betaproteobacteria & Burkholderiales & Alcaligenaceae & Achromobacter & yes & yes & no/yes \\
\hline Root 565 & Proteobacteria & Betaproteobacteria & Burkholderiales & Alcaligenaceae & Achromobacter & yes & $y^{b} s^{b}$ & no \\
\hline Root 473 & Proteobacteria & Betaproteobacteria & Burkholderiales & Comamonadaceae & Variovorax & yes & no & no \\
\hline Root 568 & Proteobacteria & Betaproteobacteria & Burkholderiales & Comamonadaceae & & no & no & no \\
\hline Root 1221 & Proteobacteria & Betaproteobacteria & Burkholderiales & Comamonadaceae & & yes & maybe & no \\
\hline Root 29 & Proteobacteria & Betaproteobacteria & Burkholderiales & Comamonadaceae & & yes & no & no \\
\hline Root 16D2 & Proteobacteria & Betaproteobacteria & Burkholderiales & Comamonadaceae & & yes & no/yes & no \\
\hline Root 209 & Proteobacteria & Betaproteobacteria & Burkholderiales & Comamonadaceae & & yes & yes & no \\
\hline Root 401 & Proteobacteria & Gammaproteobacteria & Pseudomonadales & Pseudomonadaceae & Pseudomonas & no & no & no \\
\hline Root 562 & Proteobacteria & Gammaproteobacteria & Pseudomonadales & Pseudomonadaceae & Pseudomonas & no & yes & no \\
\hline Root 9 & Proteobacteria & Gammaproteobacteria & Pseudomonadales & Pseudomonadaceae & Pseudomonas & no & yes & yes \\
\hline Root 569 & Proteobacteria & Gammaproteobacteria & Pseudomonadales & Pseudomonadaceae & Pseudomonas & no & yes & yes \\
\hline Root 68 & Proteobacteria & Gammaproteobacteria & Pseudomonadales & Pseudomonadaceae & Pseudomonas & yes & yes & no \\
\hline Root 71 & Proteobacteria & Gammaproteobacteria & Pseudomonadales & Pseudomonadaceae & Pseudomonas & yes & yes & no \\
\hline Root 179 & Proteobacteria & Gammaproteobacteria & Xanthomonadales & Xanthomonadaceae & Rhodanobacter & no & $\mathrm{no}^{\mathrm{b}}$ & no \\
\hline
\end{tabular}

a not tested: bacteria did not grow in drop inoculation experiment

${ }^{\mathrm{b}}$ induction of PER5 in differentiated zone for both WT and WER::FLS2-GFP fIs2

${ }^{c}$ Garrido-Oter et al. (2018) 
Supplemental Table S2: Primers and plasmids used for bacterial mutagenesis.

\begin{tabular}{|c|c|c|}
\hline Name & Sequence / plasmid characteristics ${ }^{a}$ & Reference \\
\hline \multicolumn{3}{|l|}{ Primers } \\
\hline fleQ-1 & CGGGATCCATTGAAGAAACCCGTGAGGC & Peter Kupferschmied (2015) \\
\hline fleQ-2 & CCCAAGCTTTAAAATCACCGCCAGGTCGCG & Peter Kupferschmied (2015) \\
\hline fleQ-3 & CCCAAGCTTTGACGCCGGTTTTTTCAAGTCTTTG & Peter Kupferschmied (2015) \\
\hline fleQ-4 & GGAATTCATTTCATGGCCATCGTCTTCGCG & Peter Kupferschmied (2015) \\
\hline flic-1 & ATAACAGGGTAATCTGAATTatgaatcagctagagcctgt & this study \\
\hline flic-2 & ccagctattacatgacgaattcctcgttg & this study \\
\hline flic-3 & attcgtcatgtaatagctggctaagctttggc & this study \\
\hline flic-4 & CCGGGTACCGAGCTCGAATTtcaggccttggcact & this study \\
\hline flic_check_F & gacttcgcagatccgtgg & this study \\
\hline flic_check_R & aactgcggtcgaagcttg & this study \\
\hline \multicolumn{3}{|l|}{ Plasmids } \\
\hline pEMG & $\begin{array}{l}\text { Expression vector; oriR6K, lacZ } \alpha \text { with two flanking I- } \\
\text { Scel sites; } \mathrm{Km}^{\mathrm{R}}, \mathrm{Ap}^{\mathrm{R}}\end{array}$ & $\begin{array}{l}\text { Martínez-García, E., and Lorenzo, V. } \\
\text { de (2011) }\end{array}$ \\
\hline pSW-2 & oriRK2, xylS, $P_{m}:: I$-scel; $\mathrm{Gm}^{\mathrm{R}}$ & $\begin{array}{l}\text { Martínez-García, E., and Lorenzo, V. } \\
\text { de (2011) }\end{array}$ \\
\hline pME8323 & $\begin{array}{l}\text { pEMG:: } \triangle \text { fleQ; suicide plasmid for the in-frame } \\
\text { deletion of fliC (PPRCHAO_1656) }\end{array}$ & Peter Kupferschmied (2015) \\
\hline pME11121 & $\begin{array}{l}\text { pEMG:: } \triangle \text { fliC; suicide plasmid for the in-frame } \\
\text { deletion of fliC (PPRCHAO_1651) }\end{array}$ & this study \\
\hline
\end{tabular}




\section{Supplemental Videos Titles}

Movie 1: Treatment of UBQ10::R-GECO1 in WT background with 1.25uM flg22 - overview

Movie 2: Treatment of UBQ10::R-GECO1 WER::FLS2-GFP fls2 with 1.25uM flg22 - overview

Movie 3: Treatment of UBQ10::R-GECO1 CASP1::FLS2-GFP fls2 with 1.25uM flg22 - overview

Movie 4: Treatment of UBQ10::R-GECO1 SHR::FLS2-GFP fls2 with 1.25uM flg22 - overview

Movie 5: Treatment of UBQ10::R-GECO1 UBQ10::FLS2-GFP fls2 with 1.25uM flg22 - overview

Movie 6: Treatment of UBQ10::R-GECO1 in WT background with 1.25uM flg22 - zoom in elongation zone

Movie 7: Treatment of UBQ10::R-GECO1 WER::FLS2-GFP fls2 with 1.25uM flg22 - zoom in elongation zone

Movie 8: Treatment of UBQ10::R-GECO1 CASP1::FLS2-GFP fls2 with 1.25uM flg22 - zoom in differentiated zone

Movie 9: Treatment of UBQ10::R-GECO1 SHR::FLS2-GFP fls2 with $1.25 \mathrm{uM} \mathrm{flg22} \mathrm{-} \mathrm{zoom} \mathrm{in}$ differentiated zone

Movie 10: Treatment of UBQ10::R-GECO1 UBQ10::FLS2-GFP fls2 with $1.25 \mathrm{uM}$ flg22 - zoom in differentiated zone 
bioRxiv preprint doi: https://doi.org/10.1101/2020.08.03.233817; this version posted August 3, 2020. The copyright holder for this preprint (which was not certified by peer review) is the author/funder. All rights reserved. No reuse allowed without permission.

\section{Figures}



(which was not certified by peer review) is the author/funder. All rights reserved. No reuse allowed without permission.

A
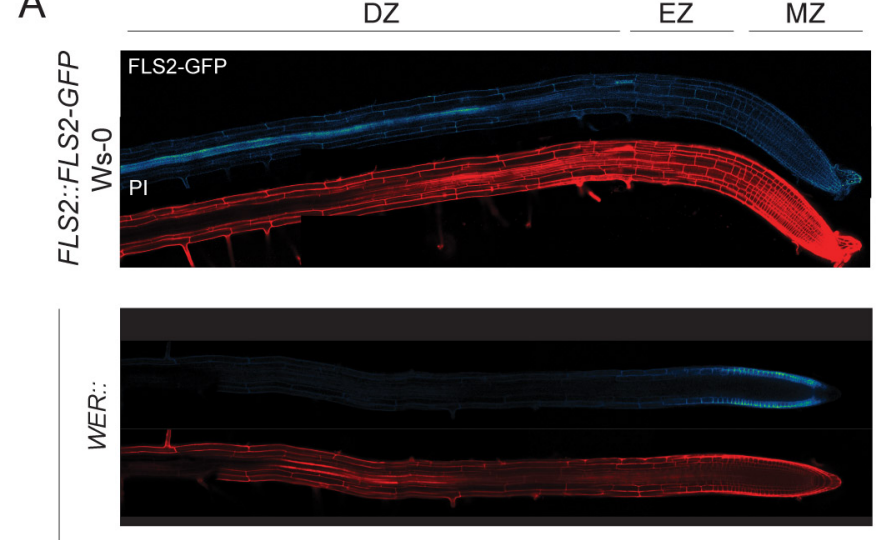

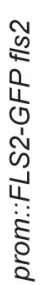
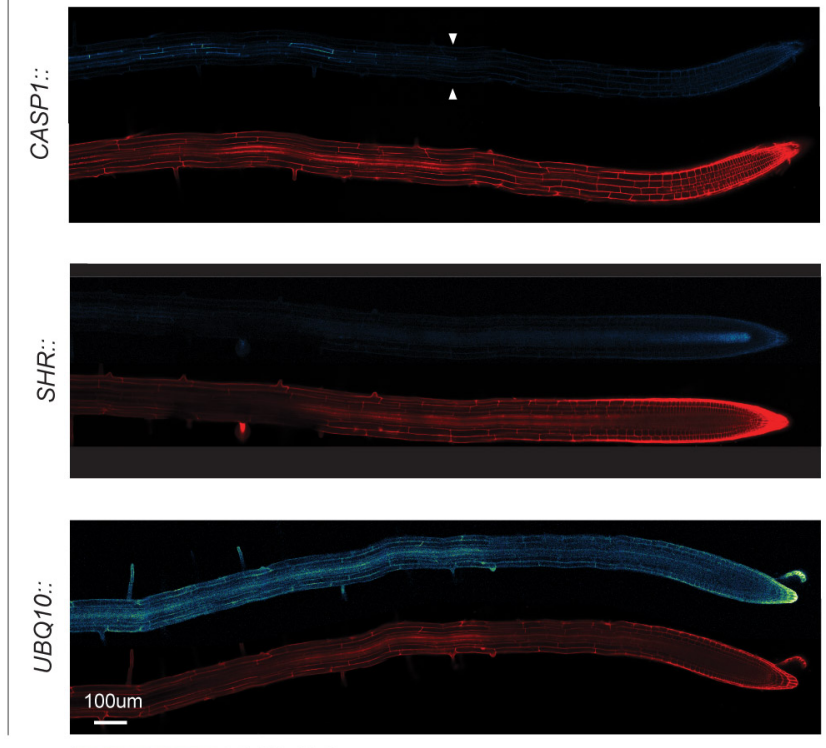

B
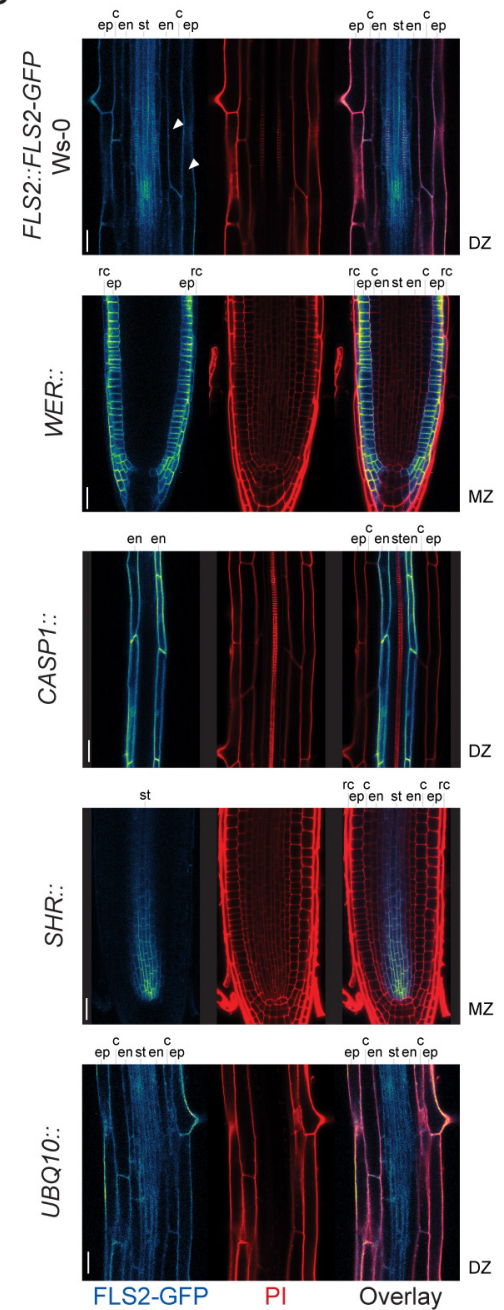

\section{Figure 1. Tissue-specific promoters drive FLS2 receptor expression ectopically}

(A) Tile scan of $f / s 2$ roots complemented with GFP-tagged FLS2 receptor under epidermal (WER::), endodermal (CASP1::), central cylinder (SHR::) and ubiquitous (UBQ10::) promoters. For comparison, endogenous FLS2 expression is shown in FLS2::FLS2-GFP Ws-0 lines. Root shape is highlighted with PI staining cell wall (PI, red). Scale bar, $100 \mu \mathrm{m}$. Developmental regions of the roots are labelled: differentiated zone (DZ), elongation zone (EZ), meristematic zone (MZ). (B) Close up view of FLS2-GFP expression at selected regions of the complemented lines. FLS2 driven by its endogenous promoter is expressed in all tissues in the differentiated zone (DZ). Note that in contrast to previous report, low FLS2 expression is observed in epidermis and cortex (white arrow). In the meristem (MZ), WER promoter expresses FLS2 specifically in epidermis (ep) and root cap (rc), SHR promoter in the stele (st) and endodermis (en). In the differentiated zone (DZ), FLS2 is expressed in all tissues under UBQ10 promoter, but is restricted to endodermis with CASP1 promoter. FLS2-GFP (BlueGreen) is co-vizualized with PI-stained cell wall (red). Separated and overlaid channels (right column) are presented. Scale bar, $25 \mu \mathrm{m}$. ep, epidermis; c, cortex; en, endodermis; st, stele; rc, root cap cells. 
bioRxiv preprint doi: https://doi.org/10.1101/2020.08.03.233817; this version posted August 3, 2020. The copyright holder for this preprint A (which was not certified by peer review) is the author/fthrbdev:LAdbrighths/seserved. No reuse allowed without permission.

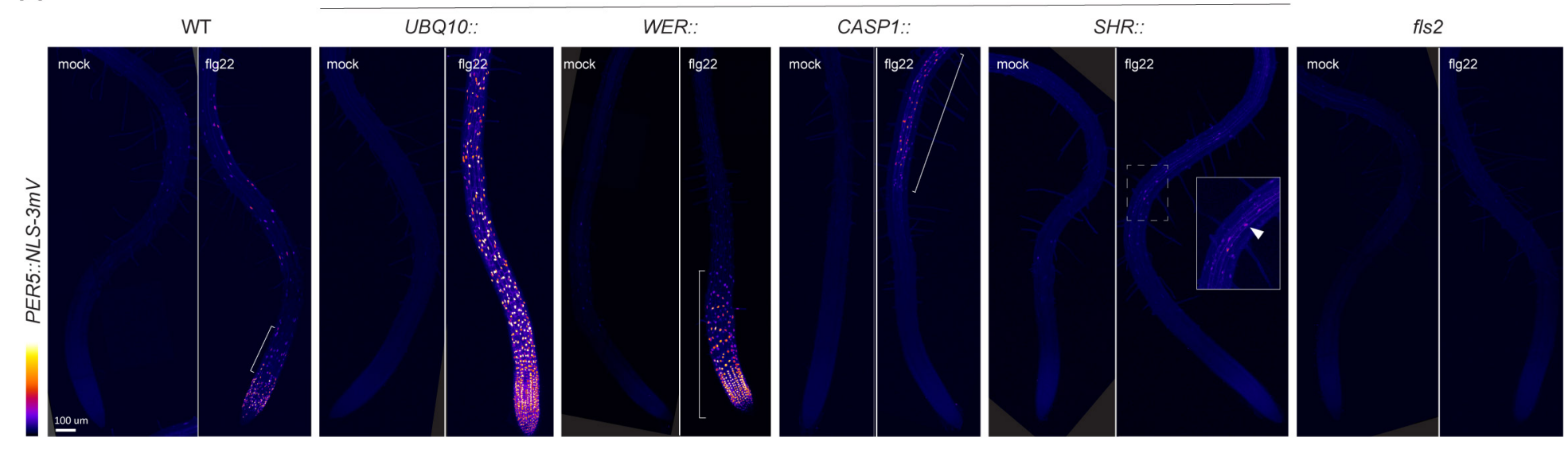

B

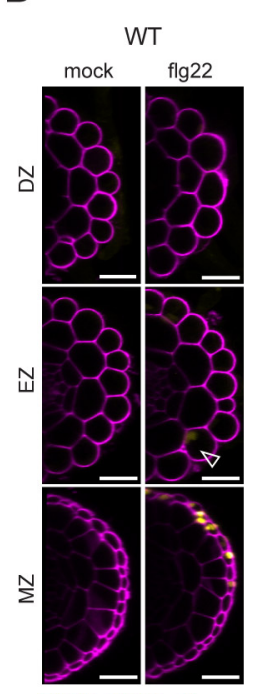

fls2

UBQ10::FLS2-GFP WER::FLS2-GFP

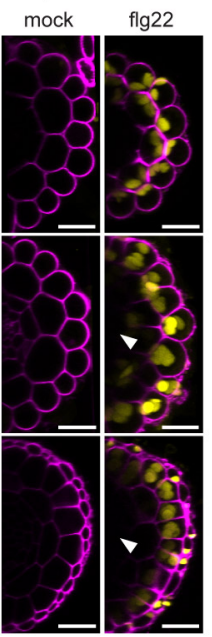

C

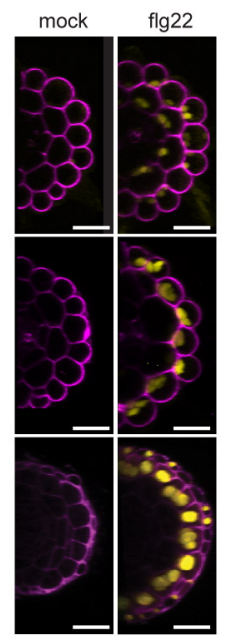

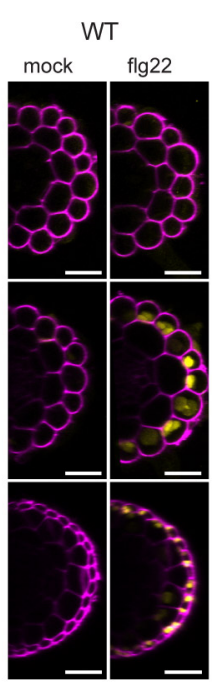

fls2

CASP1::FLS2-GFP SHR::FLS2-GFP fIs2
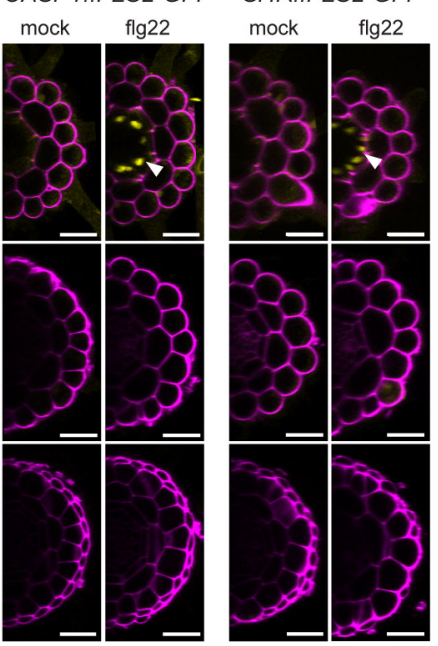

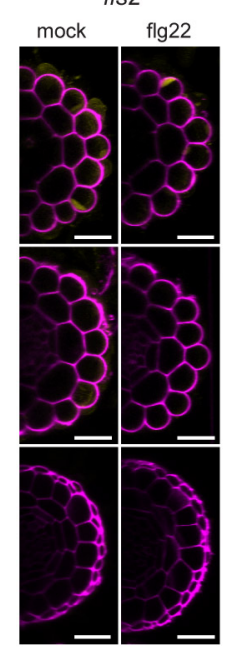

Cell wall

D

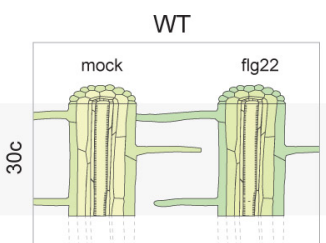

UBQ10::FLS2-GFP fIs2

WER::FLS2-GFP fls2

CASP1::FLS2-GFP fls2 SHR::FLS2-GFP fls2

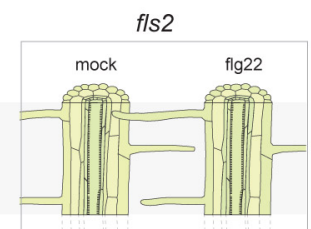

$\stackrel{0}{\simeq} N$
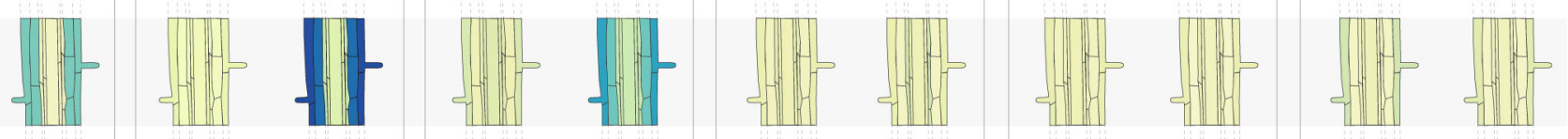

บै HAD
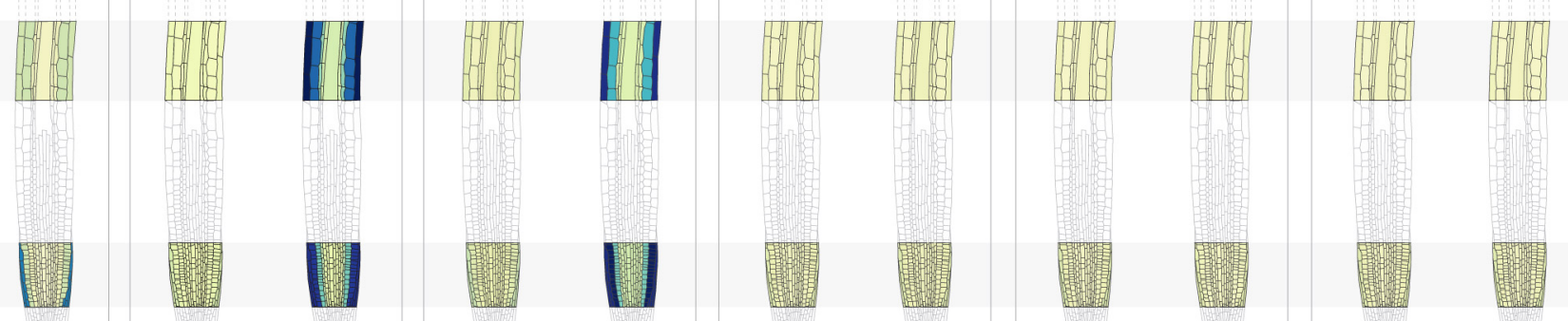

$\log _{10}$ (Mean Intensity)

$\begin{array}{llll}2.5 & 3.0 & 3.5 & 4.0\end{array}$ 


\section{Figure 2. PER5 marker gene is induced cell-autonomously by flg22 treatment}

(A) Overview of PER5::NLS-3mVenus marker response to flg22 in different FLS2 recombinant lines. Tile scan images of $1 \mu \mathrm{M}$ flg22 treated plants versus mock. Pictures were taken with similar settings. Settings were always identical between mock and corresponding flg22 treatment. Region of responsiveness is modified by the different expression patterns of $F L S 2$. Brackets indicate responsive regions. For SHR, close-up view was generated with increased brightness to highlight stellar signal (white arrow). Scale bar, $100 \mu \mathrm{m}$. (B) Maximal projection of transverse sections views of PER5 expression pattern in UBQ10:: and WER::FLS2-GFP fls2 compared to WT shown for meristematic zone (MZ), elongation zone (EZ) and differentiated zone (DZ, 30 cells after start of elongation). Seedlings were treated for $24 \mathrm{~h}$ with $1 \mu \mathrm{M}$ flg22. Note the refractory region in the central cylinder in UBQ10::FLS2 (white arrows). Nuclear localized mVenus signal (yellow) was co-displayed with propidium iodide cell wall marker (PI, purple). Images were taken with similar settings, but corresponding mock and flg22 treatment pictures for each zone separately always have identical parameters. Note that epidermal signal in flg22-treated wild-type seedlings is faint (EZ, black arrow), due to settings chosen to avoid saturation of signal in the transgenic lines. Compare to Fig.2C, WT. Scale bar, $25 \mu \mathrm{m}$. (C) Maximal projection of transverse section views of PER5::NLS-3mVenus expression pattern in CASP1:: and SHR::FLS2-GFP fls2 as well as WT and fls2 control. White arrows point at ectopic response in the endodermis. Images were acquired as in Fig.2B., with similar settings between genotypes, but with identical parameter for corresponding mock and flg22 treatment. Pictures were acquired with increased gain compared to Fig.2B due to lower average signal intensity. Scale bar, $25 \mu \mathrm{m}$. (D) Quantitative map of PER5::NLS-3mVenus responses inferred from tissue specific quantification after $24 \mathrm{~h}$ treatment with $1 \mu \mathrm{M}$ flg22. Nuclear signals were quantified in ROI delimited with UBQ10::NLSmTurquoises2 for all tissue-specific promoter lines, while wild-type (WT) signal was quantified with UBQ10::NLS-tdTomato marker. Mean intensity is therefore comparable between prom::FLS2-GFP fls2 lines, but not to wild-type. 
bioRxiv preprint doi: https://doi.org/10.1101/2020.08.03.233817; this version posted August 3, 2020. The copyright holder for this preprint (which was not certified by peer review) is the author/funder. All rights reserved. No reuse allowed without permission.

A UBQ10::R-GECO1+1.25uM flg22
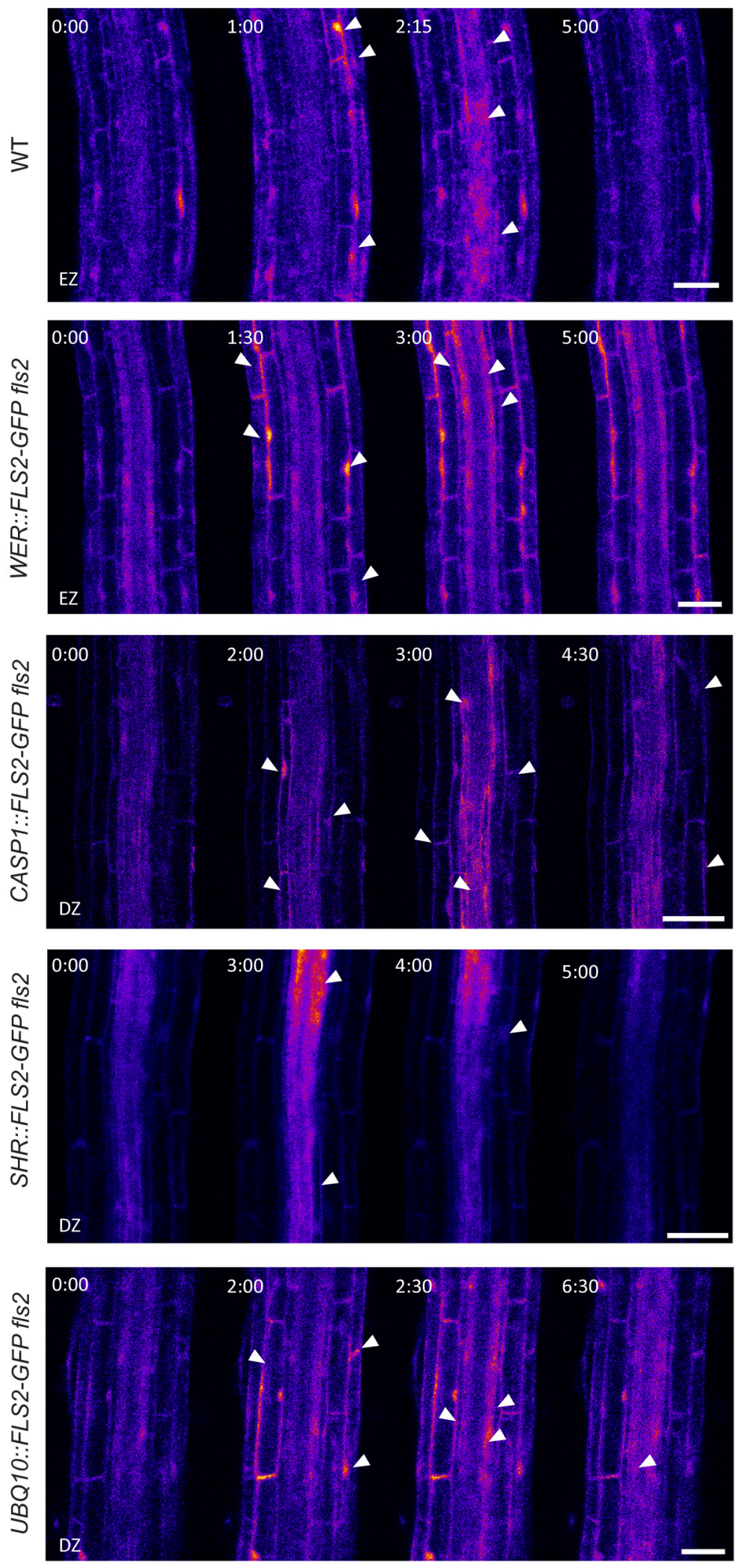

B
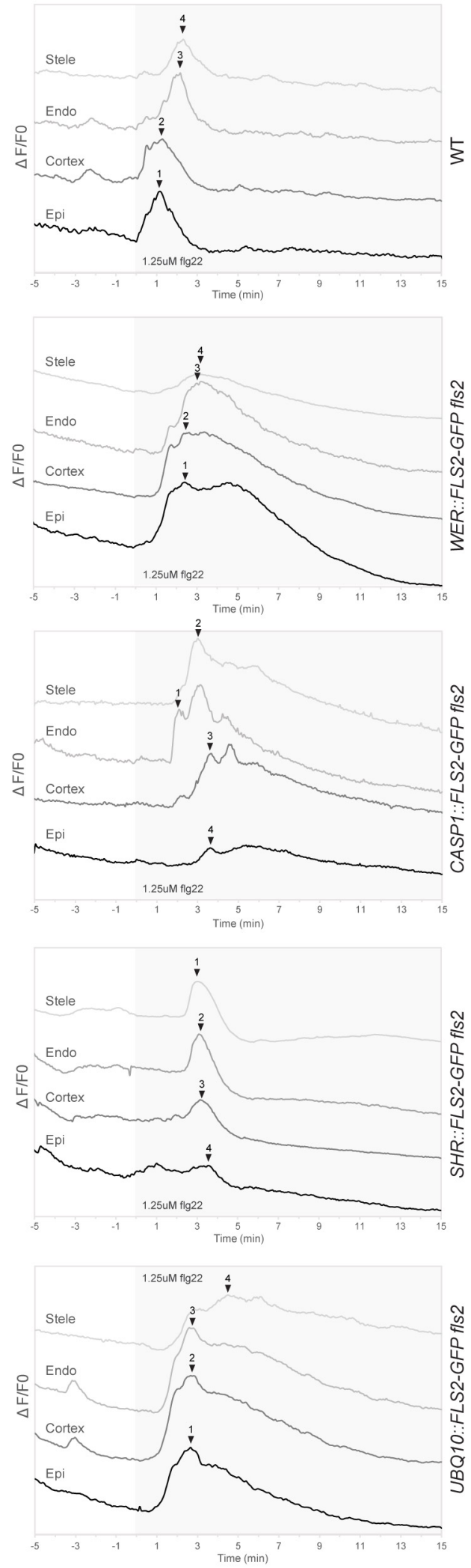

Figure 3. $\mathrm{Ca}^{2+}$ waves are non-cell autonomous responses

(A) $\mathrm{Ca}^{2+}$-dependent signal in the prom::FLS2-GFP fls2 lines in response to $1.25 \mu \mathrm{M}$ flg22. Time series of $U B Q 10:: R-G E C O 1$ fluorescence: pictures are longitudinal middle sections of roots at the elongation zone (EZ) or differentiated zone (DZ). Time 0:00 corresponds to the start of flg22 treatment. White arrows point at tissues showing a strong increase in $\mathrm{Ca}^{2+}$ content. Scale bar, $25 \mu \mathrm{m}$. (B) Normalized R-GECO1 fluorescence intensity $(\Delta F / F)$ measured in tissue-specific ROls. Values present the dynamics of $\mathrm{Ca}^{2+}$ cytosolic concentration in response to flg22 in the root shown in (A) for each tissue type. Black arrows point at the maximum intensity of the trace. Grey background corresponds to flg22 treatment. 
bioRxiv preprint doi: https://doi.org/10.1101/2020.08.03.233817; this version posted August 3, 2020. The copyright holder for this preprint (which was not certified by peer review) is the author/funder. All rights reserved. No reuse allowed without permission.

A

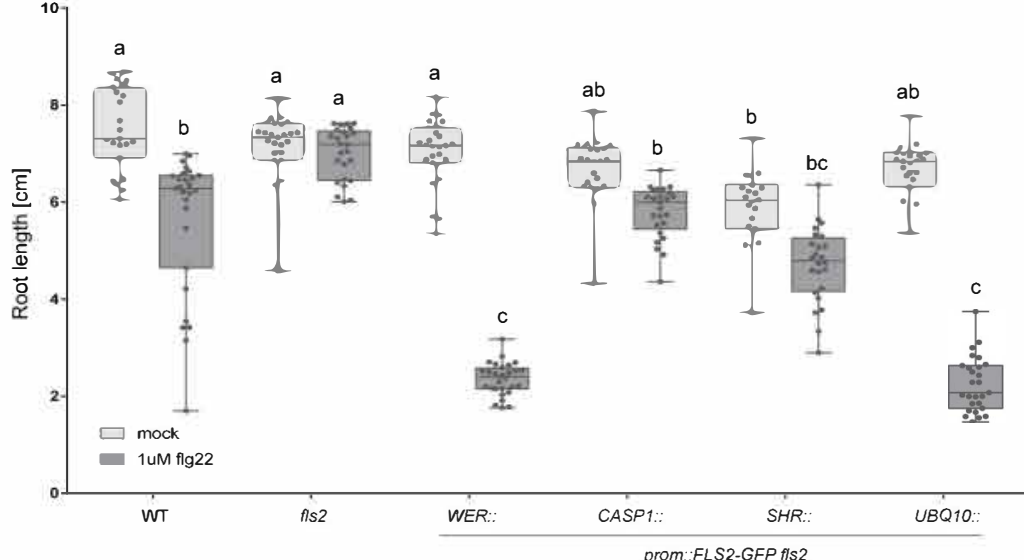

$\mathrm{B}$

prom::FLS2-GFP fls2

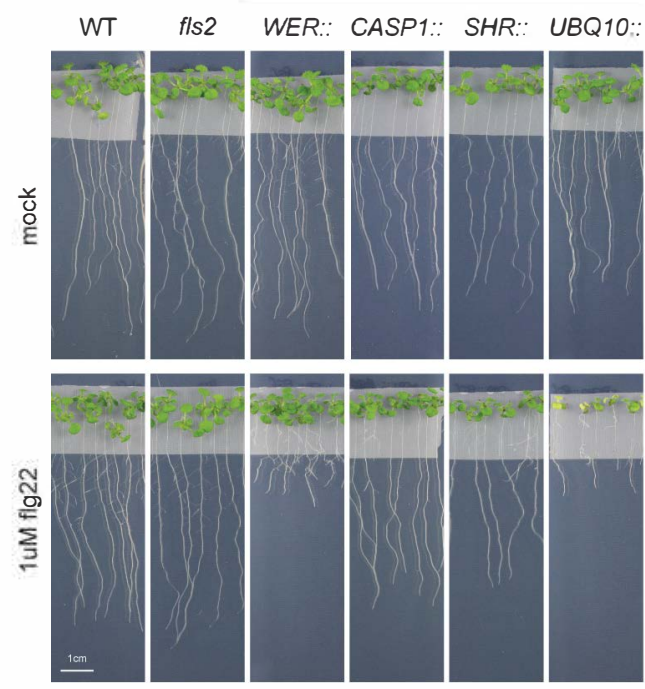

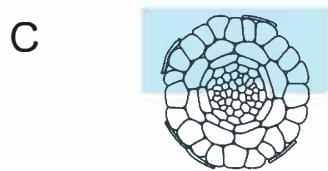
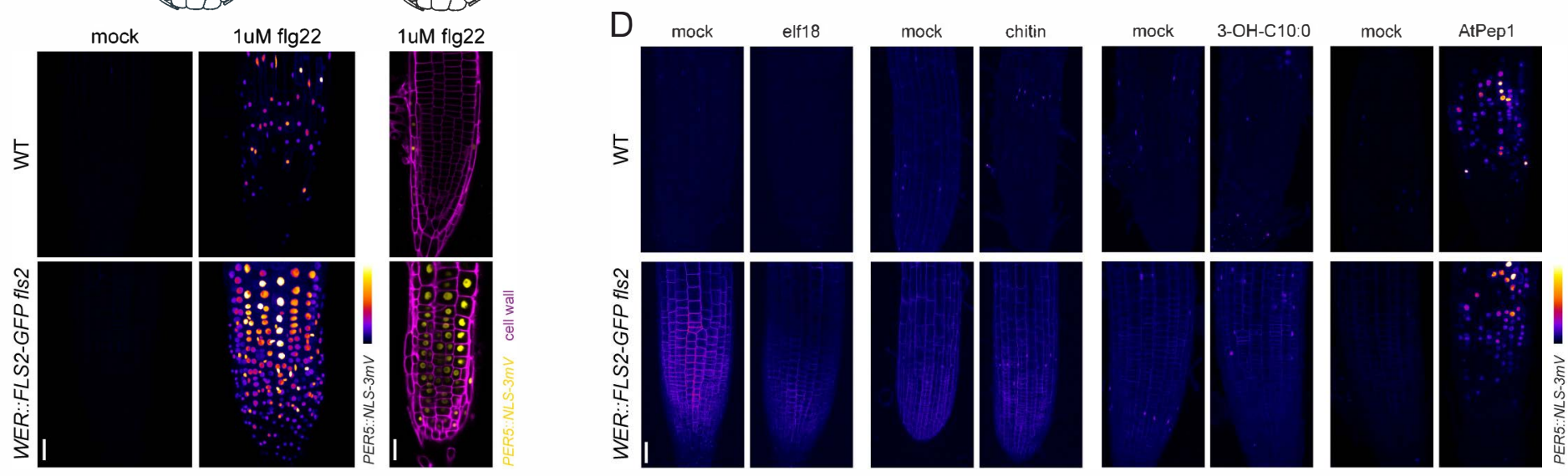

E
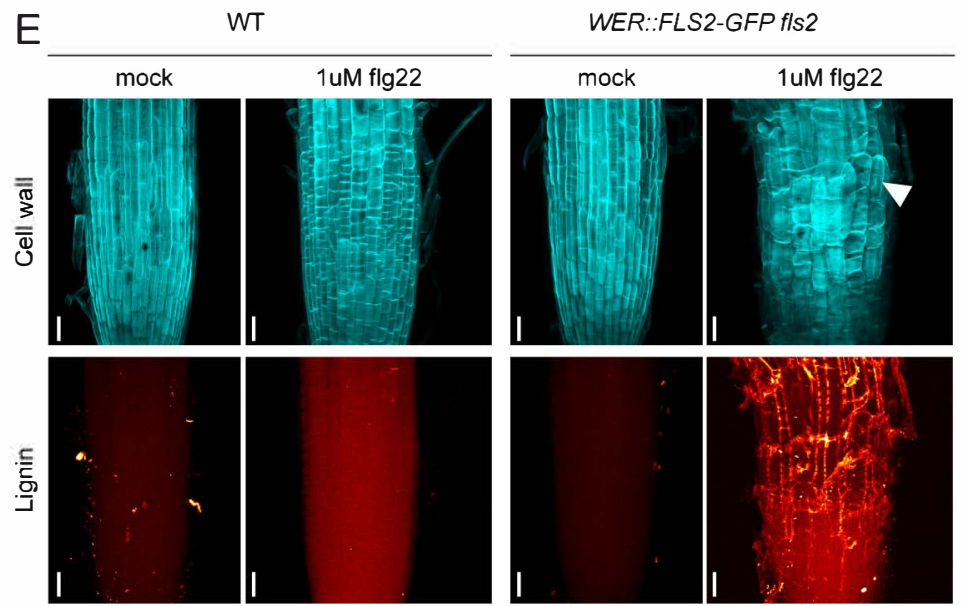

$\mathrm{F}$
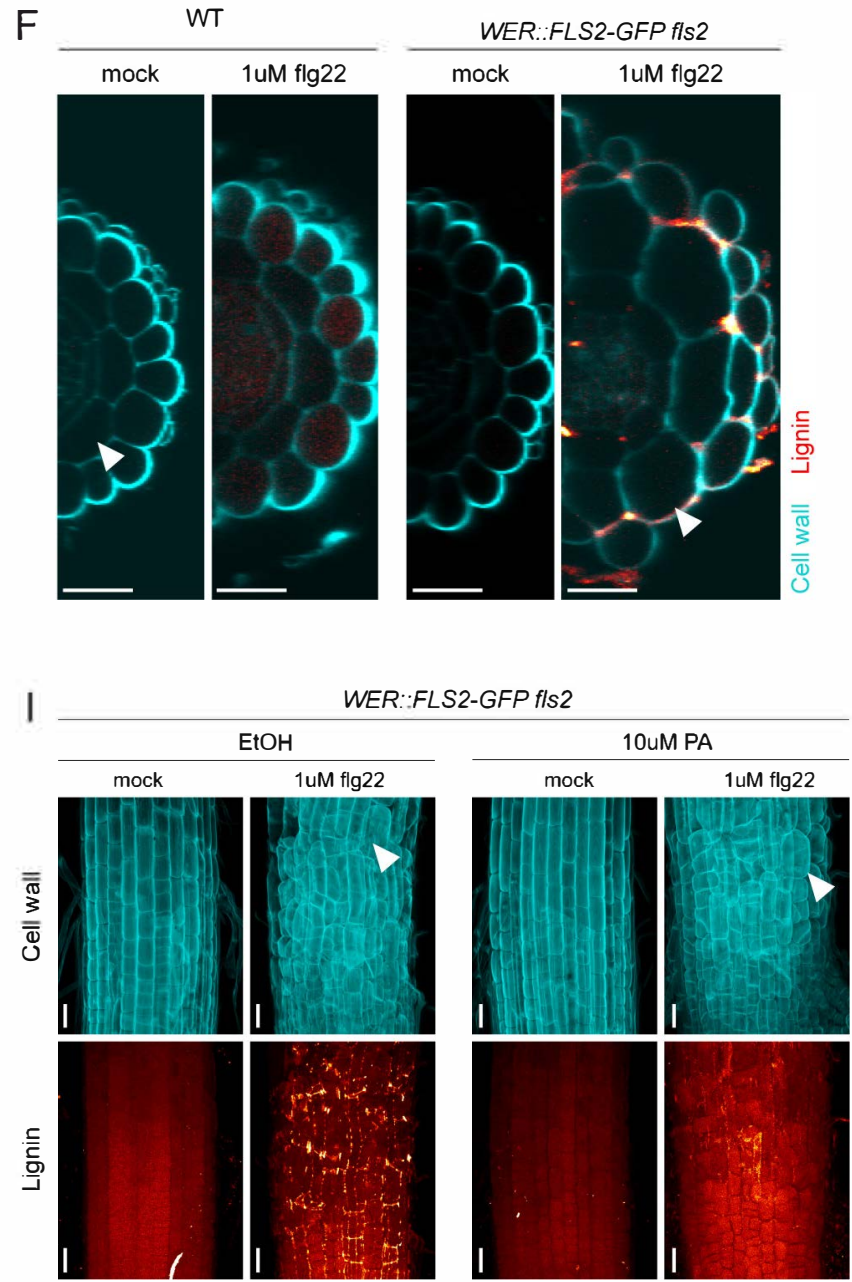
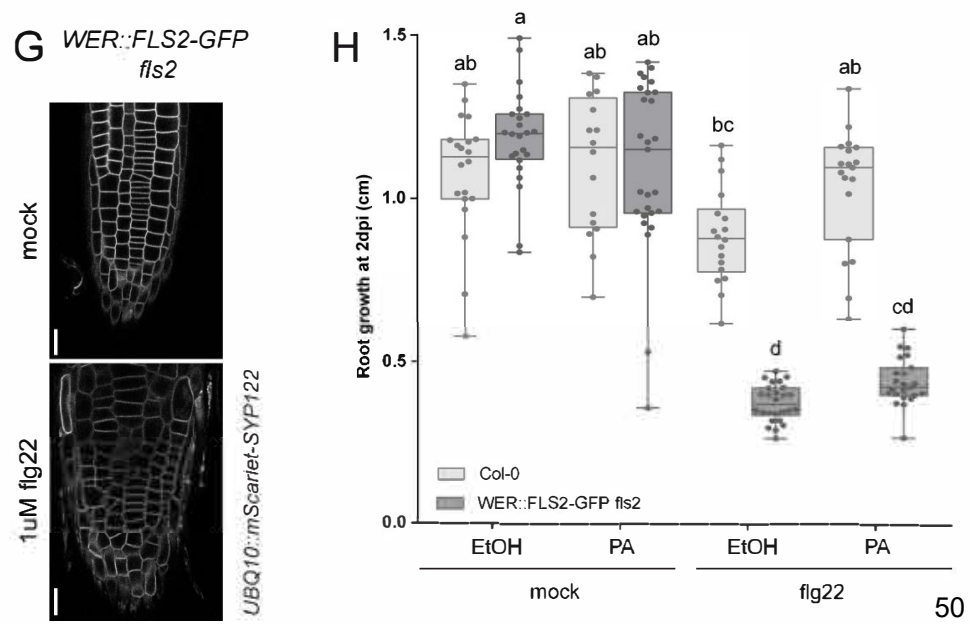
Figure 4. Epidermal meristematic expression of FLS2 leads to flg22 hypersensitivity and meristem collapse

(A) Flg22 treatment increases root growth inhibition in WER::FLS2 hypersensitive line. Root length quantification of prom::FLS2-GFP fls2 lines transferred on $1 \mu \mathrm{M}$ flg22 for $6 \mathrm{dpi}$. Boxplot centre represents the median ( $n=23$ to 28 roots). Different letters indicate statistically significant difference between means by Kruskal-Wallis test and Dunn's multiple comparison. (B) Representative pictures of seedlings transferred for 6 days on $1 \mu \mathrm{M}$ flg22. Scale bar, $1 \mathrm{~cm}$. (C) Flg22 induces strongly PER5::NLS-3mVenus in the epidermis of WER::FLS2-GFP fls2 hypersensitive line. On the right, maximum projection of zstacks taken in root tips of plants treated for $24 \mathrm{~h}$ with $1 \mu \mathrm{M}$ flg22 or mock. Schematic represents the depth of the z-stack. Pictures were taken with identical settings. Scale bar, $25 \mu \mathrm{m}$. (D) WER::FLS2-GFP fls2 hypersensitivity is specific to flg22. WER::FLS2-GFP fls2 and wild-type plants were treated for $24 \mathrm{~h}$ with either $1 \mu \mathrm{M}$ elf18, $2 \mu \mathrm{g} / \mathrm{ml}$ chitin, $1 \mu \mathrm{M}$ 3-OH-C10:0 or $1 \mu \mathrm{M}$ AtPEP1. Maximum projection of $z$-stacks taken in root tips. PER5 induction is highlighted with mVenus (Fire LUT). Parameters were identical for mock and treatment. Scale bar, $25 \mu \mathrm{m}$. (E) Treatment of WER ::FLS2-GFP fls2 for 2 days with $1 \mu \mathrm{M}$ flg22 induces meristem swelling and lignin deposition. Upper panel shows median projection of calcofluor white stained cell wall in the transition zone of the root tip (blue). Note bulky cells of the epidermis (white arrowhead). Lower panel presents maximum projection of lignin deposition stained with basic fuchsin (red). Lignin accumulates between cells only in WER::FLS2-GFP fls2 after flg22 treatment. Scale bar, $25 \mu \mathrm{m}$. $(\mathbf{F})$ Cross-section of pictures in $(E)$. Cell wall stained with calcofluor white (blue) is covisualized with lignin stained with basic fuchsin (red). Flg22 treatment induces massive swelling of cortex cells (white arrowheads) only in WER::FLS2-GFP fls2. Lignin is principally deposited between epidermal and cortex cells. Epidermal cells are pushed apart by the swelling cortex and are sometimes missing. Scale bar, $25 \mu \mathrm{m}$. (G) Epidermal view of plasma membrane visualized by the construct UBQ10::mScarlet-SYP122 in WER::FLS2-GFP fls2. Cell division is disorganized after $1 \mu \mathrm{M}$ flg22 treatment. Scale bar, $25 \mu \mathrm{m}$. (H) Inhibition of monolignol synthesis does not rescue meristem flg22driven increased root growth inhibition of WER::FLS2-GFP fls2. Root growth measured after overnight pre-treatment with 10uM PA inhibitor followed by $36 \mathrm{~h} 1 \mathrm{uM}$ flg22 combined to 10uM PA treatment. Boxplot centre represents the median ( $n=16$ to 27 seedlings). Different letters indicate statistically significant difference $(p<0.05)$ between means by Kruskal-Wallis test and Dunn's multiple comparison. (I) Flg22 induces meristem swelling despite inhibition of monolignol by PA treatment. Pictures taken from samples quantified in $(\mathrm{H})$. Upper panel shows median projection of calcofluor white stained cell wall in the transition zone of root tip (blue). Lower panel presents maximum projection of lignin deposition stained with basic fuchsin (red). White arrowheads points at examples of bulky cells. Scale bar, $25 \mu \mathrm{m}$. 
bioRxiv preprint doi: https://doi.org/10.1101/2020.08.03.233817; this version posted August 3, 2020. The copyright holder for this preprint (which was not certified by peer review) is the author/funder. All rights reserved. No reuse allowed without permission.

A

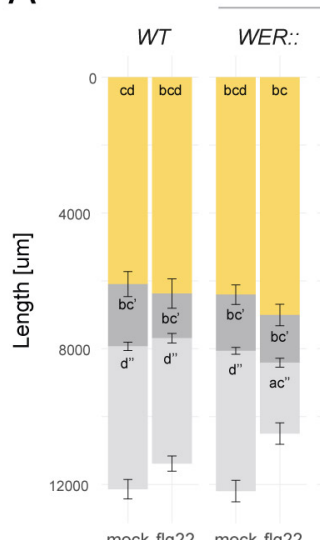

mock flg22 mock flg22 mock flg22 mock flg22 mock flg22 mock flg22
B

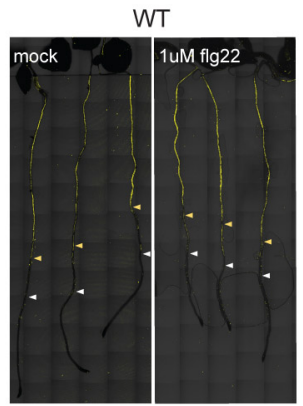

CASP1::FLS2-GFP fls2

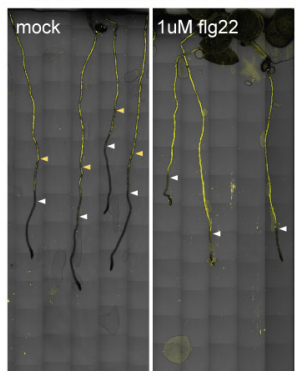

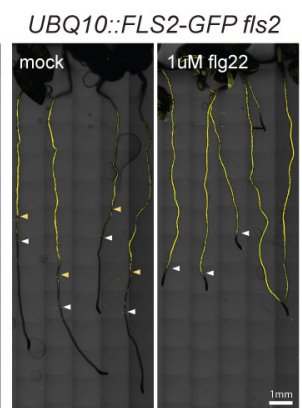

Figure 5. Suberin deposition is triggered by flg22 when endodermal cells expressed FLS2

(A) Quantification of suberized zone length in seedlings treated for 1 day with $1 \mathrm{uM}$ flg22 $(18<=\mathrm{n}<=27)$. Data of two replicates were pooled. Roots regions were classified as suberized, patchy and unsuberized zones. Error bars represent standard error (SE). Different letters indicate statistically significant differences amongst lines for the specified zone $(p<0.05)$. Multiple comparison was performed using ANOVA and Tukey's tests for the suberized zone, whereas Kruskal-Wallis and Dunn's tests were used multiple comparison of patchy and non-suberized zones. (B) Whole root views of suberin lamellae deposition in CASP1:: and UBQ10::FLS2-GFP fls2 lines compared to wild-type after $1 \mu \mathrm{M}$ flg22 treatment vs mock. Suberin was stained with fluorol yellow. White arrowheads start of patchy zone; yellow arrowheads, start of fully suberized zones. Scale bar, $1 \mathrm{~mm}$. 
bioRxiv preprint doi: https://doi.org/10.1101/2020.08.03.233817; this version posted August 3, 2020. The copyright holder for this preprint (which was not certified by peer review) is the author/funder. All rights reserved. No reuse allowed without permission.

A
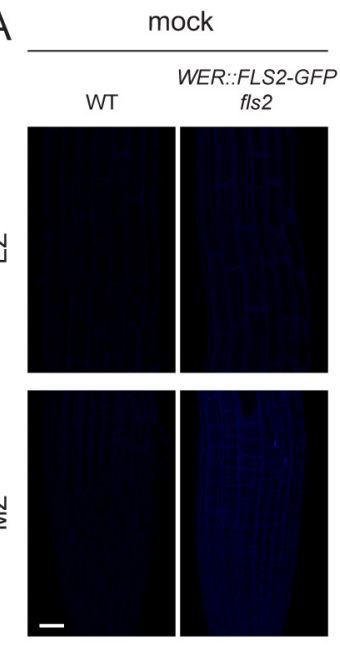
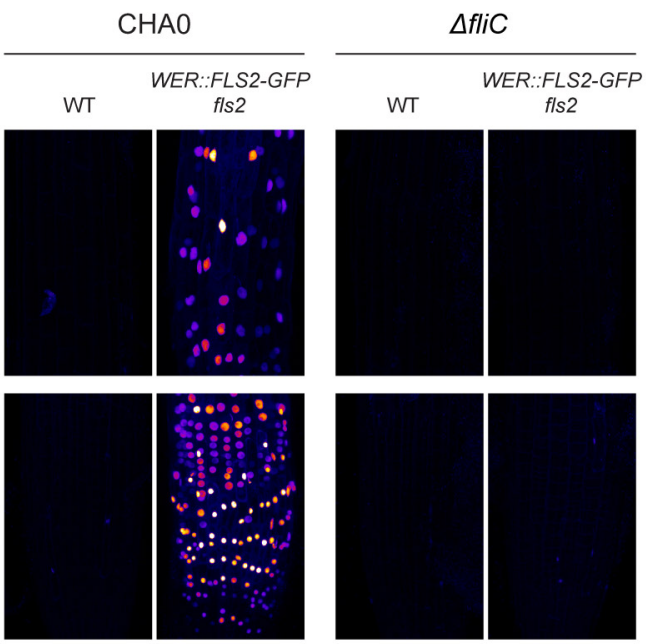
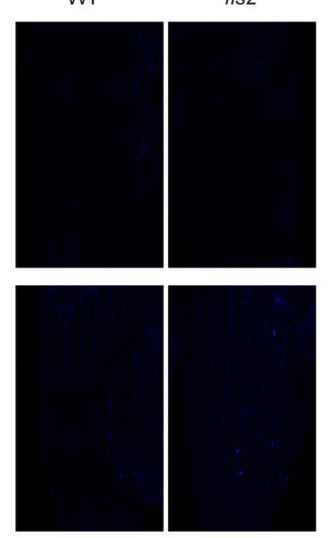

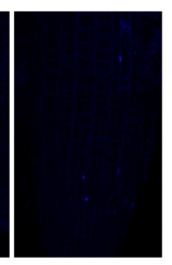

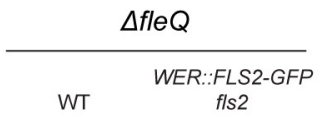
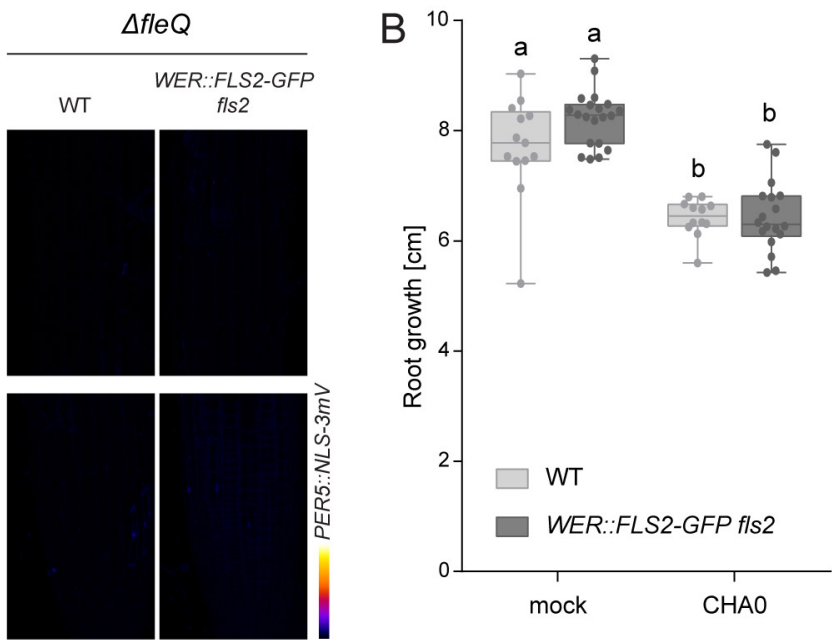

C

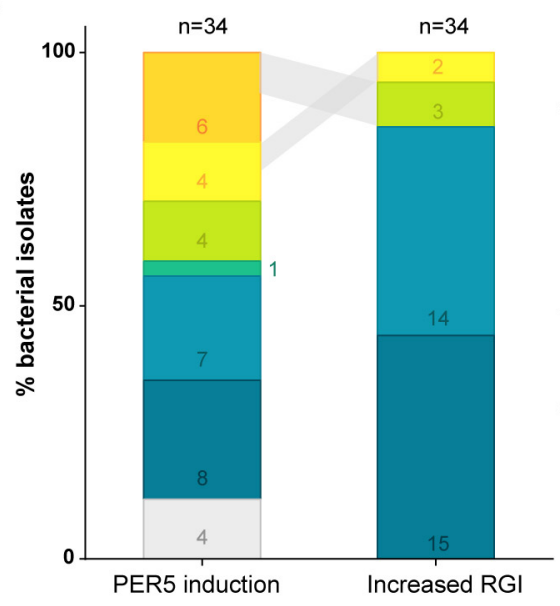

yes

$\square$ flg22 predicted

flg22 not predicted

variable

$\square$ flg22 predicted

- flg22 not predicted

no

flg22 predicted

flg22 not predicted

not tested

$\square$ flg22 not predicted

D

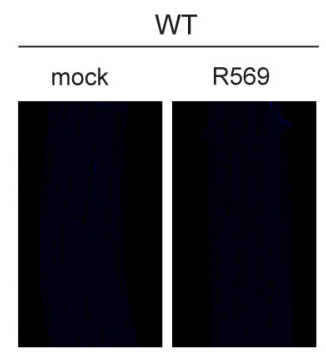

WER::FLS2-GFP fls2
mock
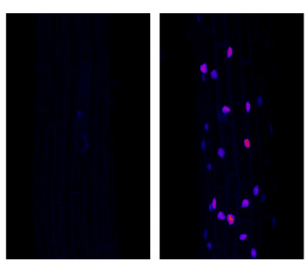

$\Sigma$
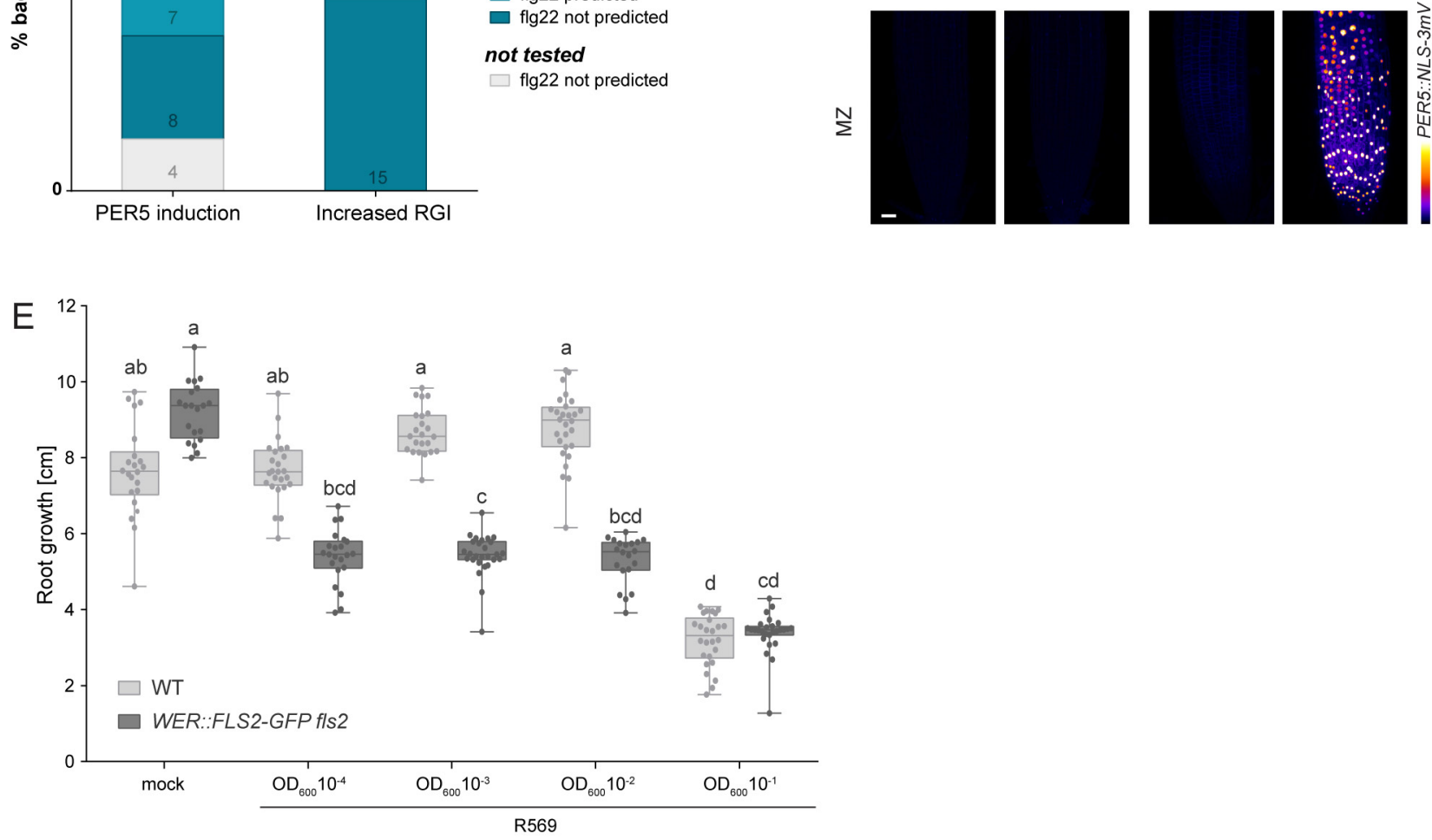


\section{Figure 6. WER::FLS2 line detects endogenous bacterial flg22}

(A) CHAO bacteria trigger a strong induction of PER5::NLS-3mVenus marker (Fire LUT) on WER::FLS2-GFP fls2. Mutants $\triangle$ fliC and $\triangle$ fleQ defective for flagellum lose their ability to induce detectable MTI. $\triangle$ fliC mutant was confirmed by motility assay (see Fig.S7D). Maximum projection of zstacks imaging meristematic (MZ) and elongation (EZ) zones treated with drop inoculation of bacterial solution of a concentration of $\mathrm{OD}_{600}=0.01$ or mock, respectively. Images were acquired at $1 \mathrm{dpi}$. Acquisition done with identical settings. Scale bar, $25 \mu \mathrm{m}$. (B) CHAO do not induce consistently increased root growth inhibition in WER::FLS2-GFP fls2. Root growth was quantified at 6 dpi on plate inoculated with bacteria at $\mathrm{OD}_{600}=10^{-3}$. Different letters indicate statistically significant differences $(p<0.05)$. Multiple comparison was performed using ANOVA and Tukey's test. (C) Proportion of natural isolates from At-SPHERE culture collection triggering stronger PER5::NLS-3mVenus induction and increased root growth inhibition (RGI) on WER::FLS2-GFP fls2 compared to wild-type seedlings (yes), or not (no). Bacteria classified in "variable" presented contradictory results between replicates. Bacteria flg22 sequence was predicted to be recognized by FLS2 (flg22 predicted) or not (flg22 not predicted). Numbers of bacterial isolates in each category are indicated in colour. Grey surfaces indicate identical bacteria strains. (D) Pseudomonas isolate R569 from At-SPHERE culture collection triggers strong PER5::NLS-3mVenus (Fire LUT ) induction on WER::FLS2-GFP fls2. Seedlings were imaged after one-day treatment with $\mathrm{OD}_{600}=0.01$. Maximum projection of $z$-stacks

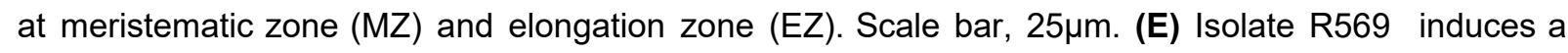
robust increased root growth inhibition on WER::FLS2-GFP fls2 compared to wild-type plants. High concentration of bacteria $\left(\mathrm{OD}_{600}=0.1\right)$ is deleterious to both genotypes. Root growth was quantified at $6 \mathrm{dpi}$ on plate inoculated with bacteria at $\mathrm{OD}_{600}=10^{-1}$ to $10^{-4}$. Different letters indicate statistically significant differences $(p<0.05)$. Multiple comparison was performed using Kruskal-Wallis and Dunn's test. 
bioRxiv preprint doi: https://doi.org/10.1101/2020.08.03.233817; this version posted August 3, 2020. The copyright holder for this preprint (which was not certified by peer review) is the author/funder. All rights reserved. No reuse allowed without permission.

\section{Supplemental Figures}


bioRxiv preprint doi: https://doi.org/10.1101/2020.08.03.233817; this version posted August 3, 2020. The copyright holder for this preprint (which was not certified by peer review) is the author/funder. All rights reserved. No reuse allowed without permission.

A

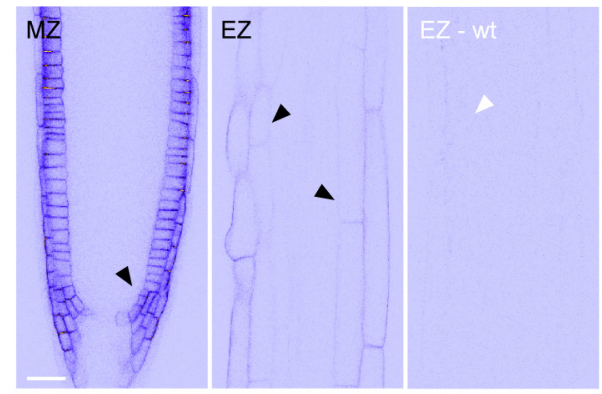

D

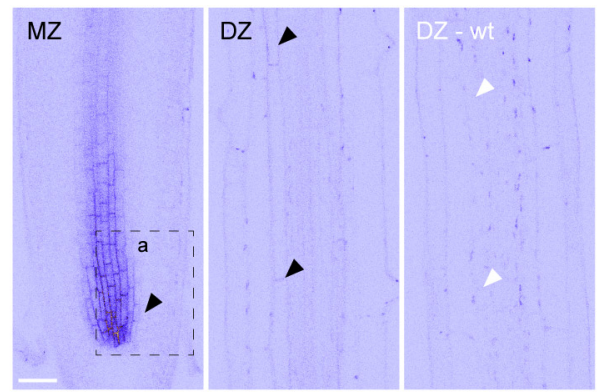

$E$

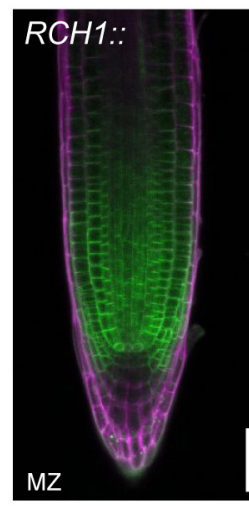

prom::FLS2-GFP fls2
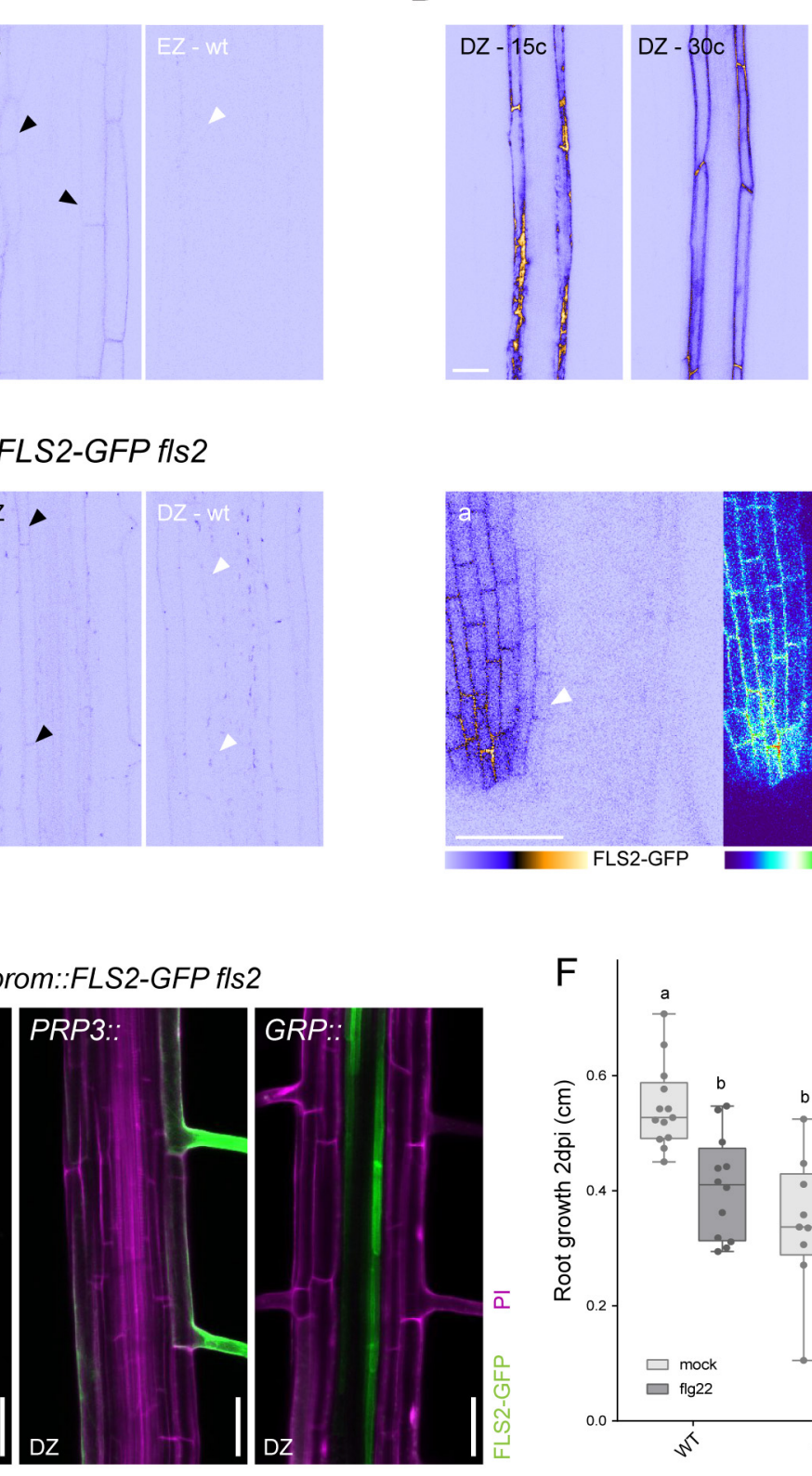

C

UBQ10::FLS2-GFP fls2
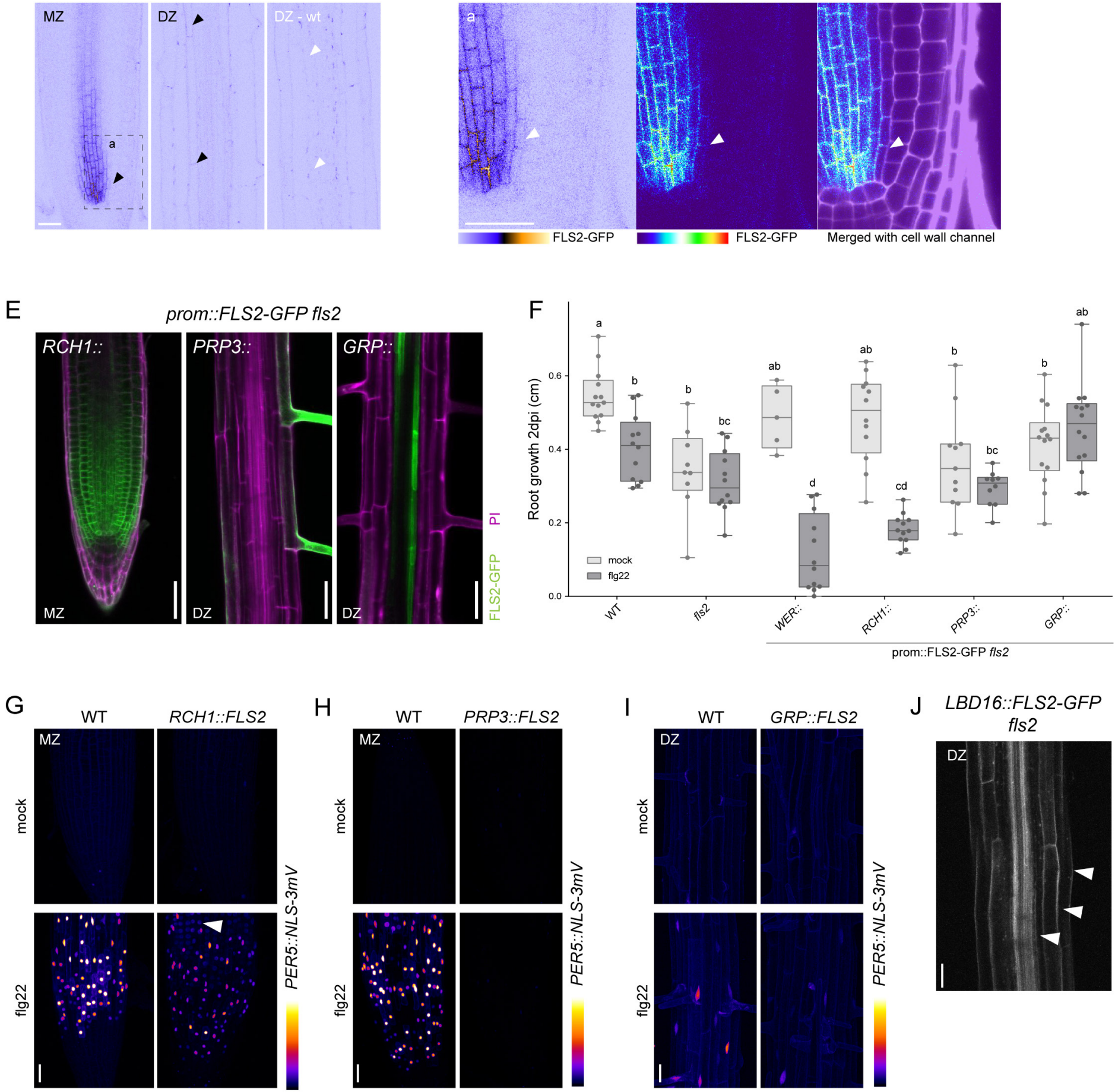

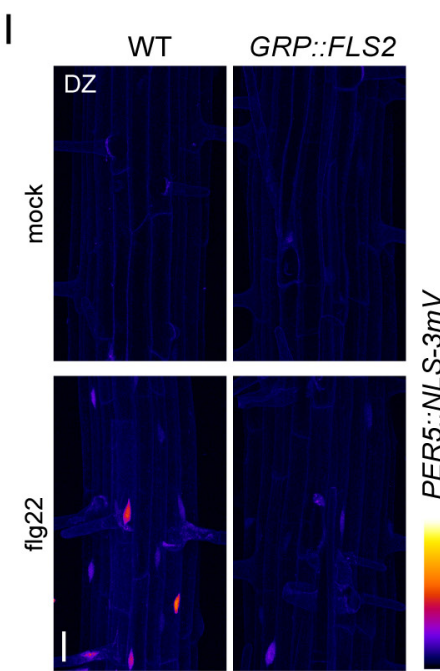

J LBD16::FLS2-GFP

fls2

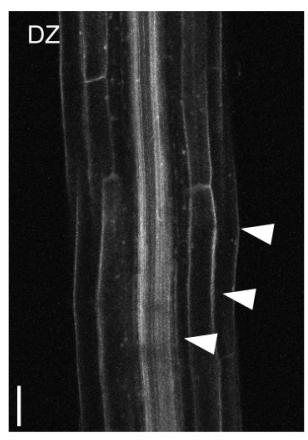




\section{Supplemental Figure S1. Expression pattern of prom::FLS2 complementing fls2.}

(A) WER::FLS2-GFP expression. WER promoter expressed principally FLS2 in epidermal cells, but some weak signal can be observed in cortex (black arrowheads). Picture of wild-type plants taken with identical setting (EZ-wt) is showed for comparison (cortical cell, white arrowhead). (B) CASP1::FLS2$G F P$ is expressed exclusively in endodermal cell line in early and later differentiated zones (15 cells respectively 30 cells after onset of elongation). (C) UBQ10::FLS2-GFP is expressed in all tissue types in every region of the root. (D) SHR::FLS2-GFP is expressed strongly in the stele of the meristem then decreases in intensity in later regions. Some weak signal can be detected in endodermal cells (black arrowheads). Picture of wild-type plants taken with identical setting (DZ-wt) is shown for comparison (endodermal cells, white arrowheads). Close-up view of dashed squared box is found in (a). FLS2-GFP (visualized by ICA and Thermal LUTs) is merged with cell wall stained by PI (white). White arrowheads point at endodermal cells expressing weakly $F L S 2$. (E) $R C H 1$ promoter expresses $F L S 2$ in the meristem, $P R P 3::$ in the root hair cells and GRP::: in the pericycle cells. FLS2-GFP (green) is co-visualized with PIstained cell wall (magenta). (F) Flg22 treatment increases root growth inhibition in WER::FLS2 and $R C H 1:: F L S 2$ hypersensitive line only. Root length quantification of prom::FLS2-GFP fls2 lines treated with $1 \mu \mathrm{M}$ flg22 for 2 days. Boxplot centre represents the median ( $n=5$ to 14 roots). Different letters indicate statistically significant difference between means by 2-ways ANOVA and Tukey multiple comparison. (G) Maximal projection PER5::NLS-3mVenus marker (Fire LUT) in RCH1::FLS2-GFP fls2 compared to WT shown for MZ. Seedlings were treated for $24 \mathrm{~h}$ with $1 \mu \mathrm{M}$ flg22. Images were taken with identical settings. White arrow, epidermal signal. (H) Maximal projection PER5::NLS-3mVenus marker (Fire LUT) in PRP3::FLS2-GFP fls2 compared to WT shown for MZ. Seedlings were treated for $24 \mathrm{~h}$ with $1 \mu \mathrm{M}$ flg22. Images were taken with identical settings. (I) Maximal projection PER5::NLS-3mVenus marker (Fire LUT) in GRP::FLS2-GFP fls2 compared to WT shown for the DZ. Seedlings were treated for $24 \mathrm{~h}$ with $1 \mu \mathrm{M}$ flg22. Images were taken with identical settings. (J) LBD16:: promoter expresses FLS2GFP in all tissues in the differentiated zone (DZ). Note that in contrast to previous report, FLS2 is present in epidermis, cortex and endodermis (white arrows) in addition to the stele. Meristematic zone (MZ), elongation zone (EZ), differentiation zone (DZ). Scale bar, $25 \mu \mathrm{M}$. 
bioRxiv preprint doi: https://doi.org/10.1101/2020.08.03.233817; this version posted August 3, 2020. The copyright holder for this preprint (which was not certified by peer review) is the author/funder. All rights reserved. No reuse allowed without permission.

A
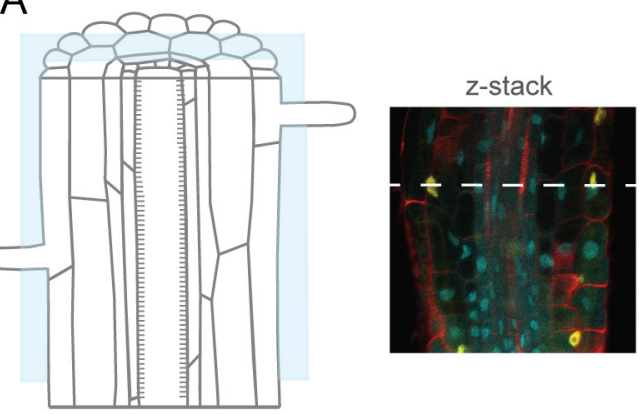

reslice

E

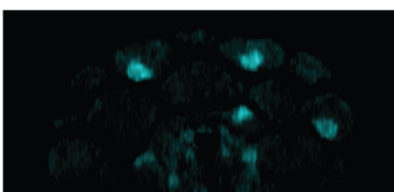

transform to 8 bits 3D iterative thresholding

$\mathrm{F}$

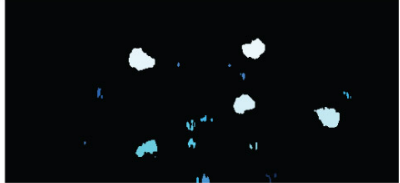

Threshold

$J$

Heat map generation
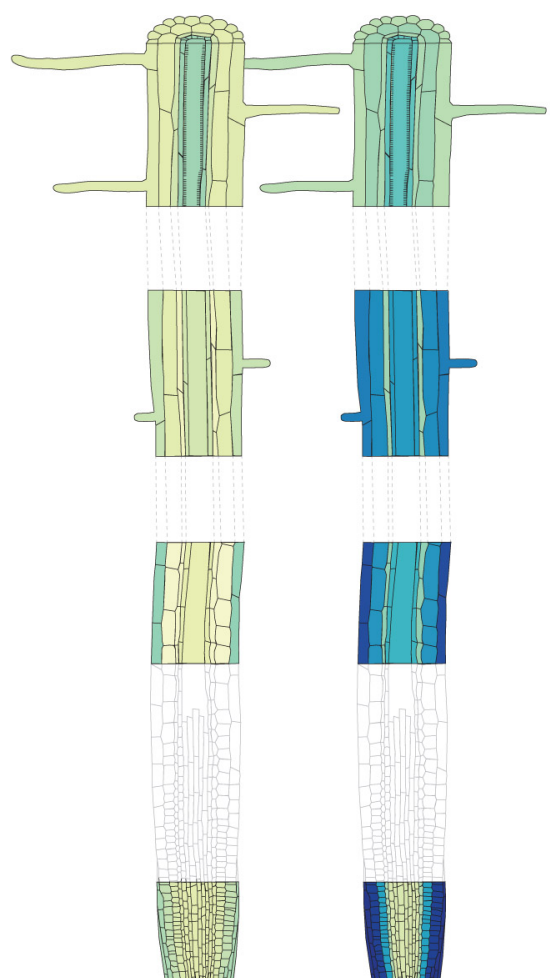

G

\section{D object counter}

define ROIs from threshold

redirect analysis on PTI marker channel

Gaussian

blurr
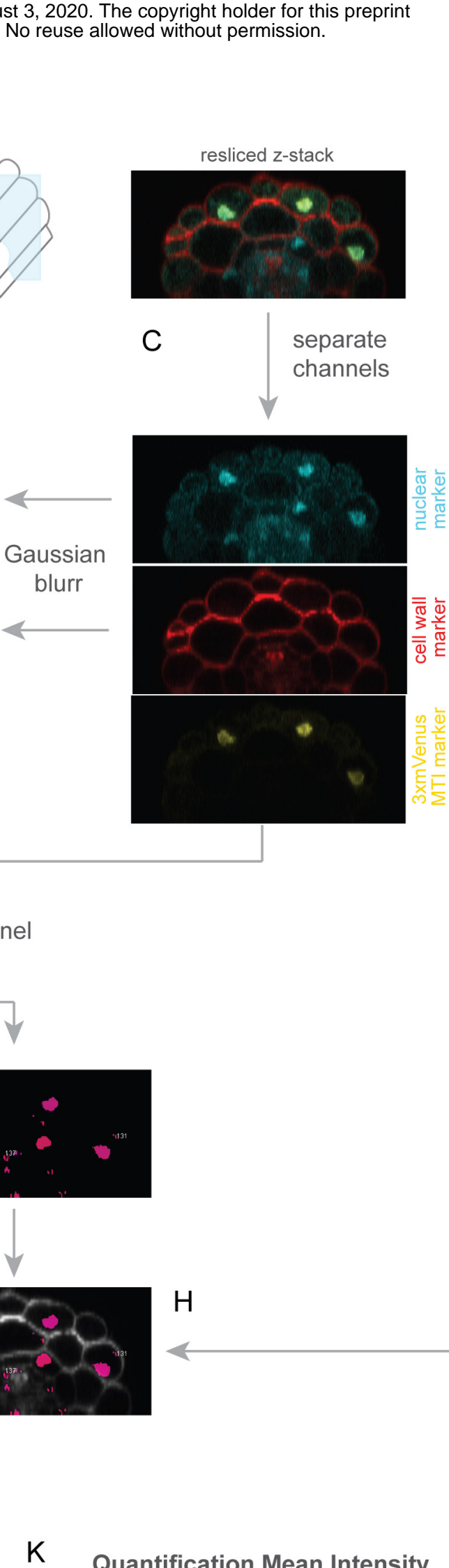

resliced z-stack
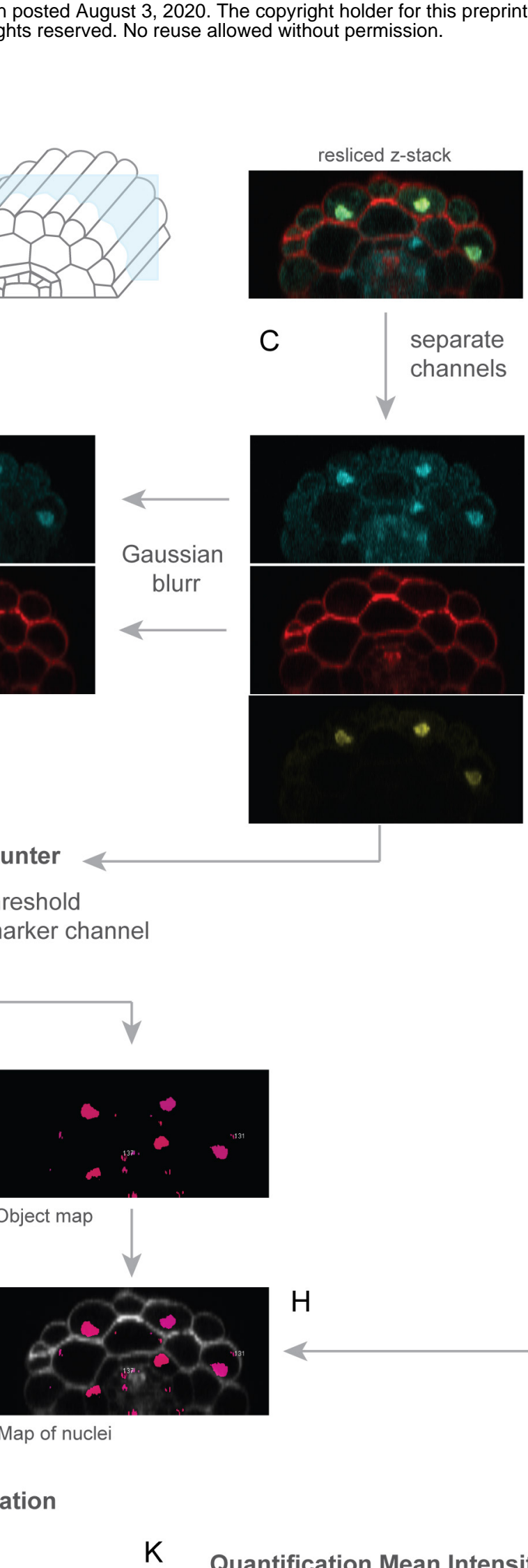

Object map
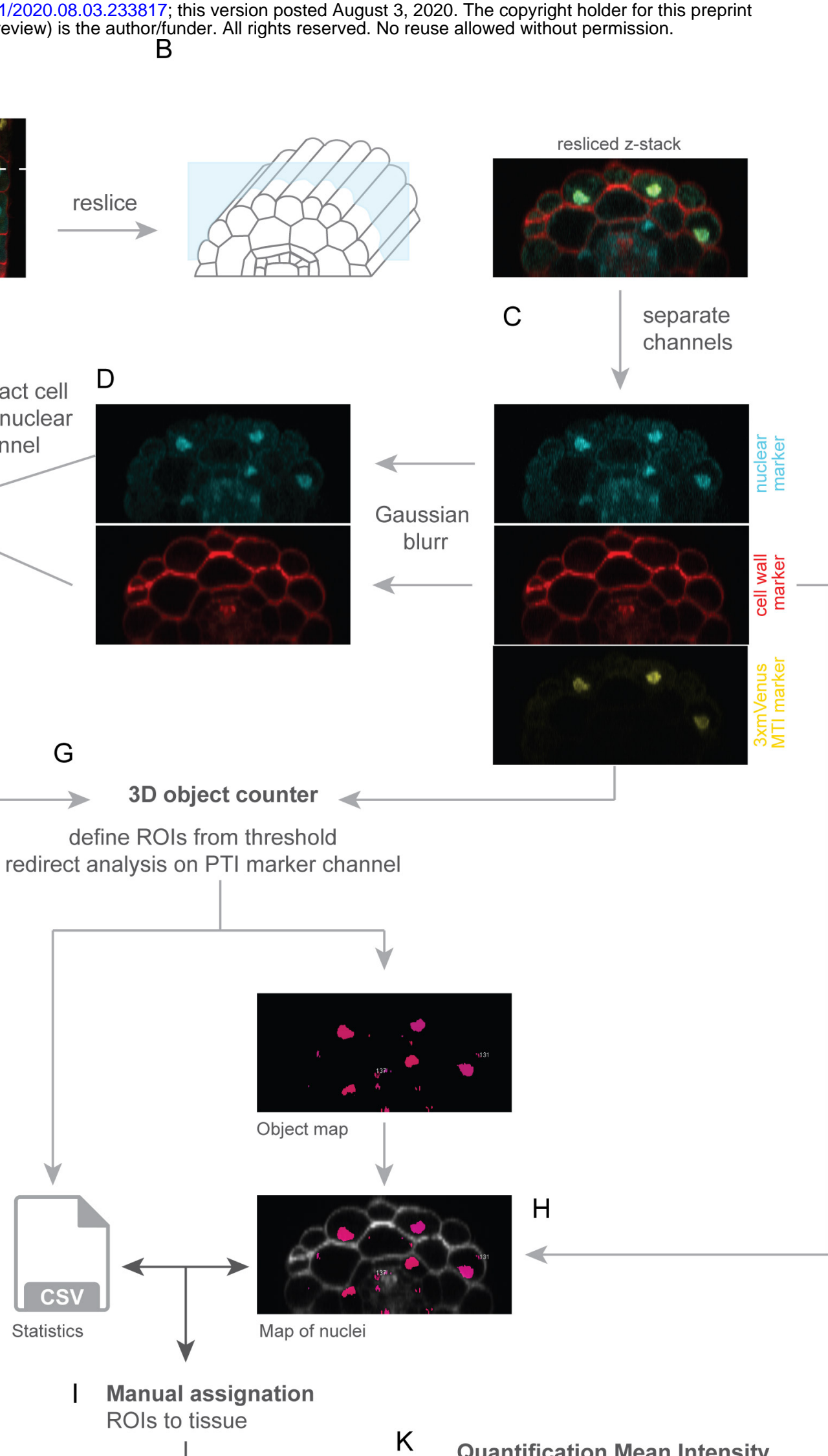

K

Quantification Mean Intensity for each tissue

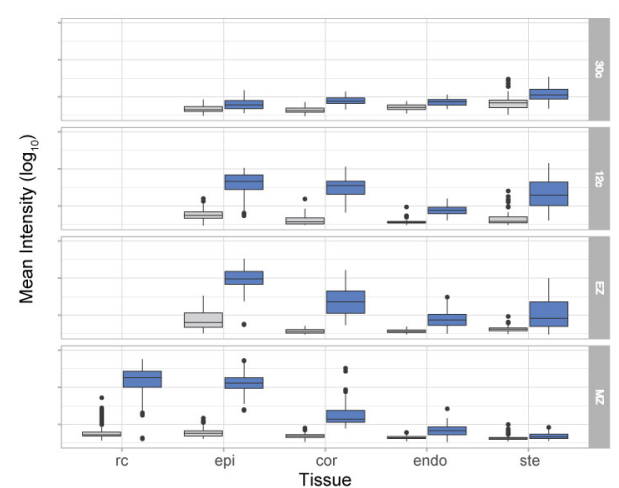




\section{Supplemental Figure S2. Quantification procedure}

(A) Z-stack images with 3 channels (red: cell wall, blue: UBQ10::NLS-mTurquoise/tdTomato, yellow: PER5/MYB51::NLS-3mVenus) were taken from 4 different regions of the root (meristematic zone, elongation zone, 12 cells and 30 cells after onset of elongation), for 3 to 6 seedlings by treatment by genotype. (B) Each Z-stacks are resliced to get cross-section view. The three channels are separated (C) and a Gaussian blur filter is applied on the cell wall and the MTI marker channel (D). Blurred cell wall channel is then subtracted from blurred nuclear marker channel to remove non-nuclear background (E). The obtained cleaned nuclear channel is then converted to 8-bit and a 3D iterative thresholding is performed to delimit ROI for each nuclei (F). The 3D object counter plugin is then used to measure the mean signal intensity of each nuclei delimited by the obtained ROIs in the MTI marker channel. The plugin gives as output a .csv file with the measured values, a masked image of the PTI marker channel and an object map, delimiting the identified nuclei (G). The object map is then coupled to the original cell wall marker to define the tissue origin of each nuclei $(\mathbf{H})$. Each map was then reviewed manually to assign 20 nuclei for each cell type and to complete .csv files (I). Average of the mean signal intensity of each nuclear tissue-specific signal were calculated, transformed into log10 and colour coded using the heatmaply() function in $R(\mathrm{~J})$. Boxplots were generated to represent signal variability (K). 
bioRxiv preprint doi: https://doi.org/10.1101/2020.08.03.233817; this version posted August 3, 2020. The copyright holder for this preprint (which was not certified by peer review) is the author/funder. All rights reserved. No reuse allowed without permission.

A
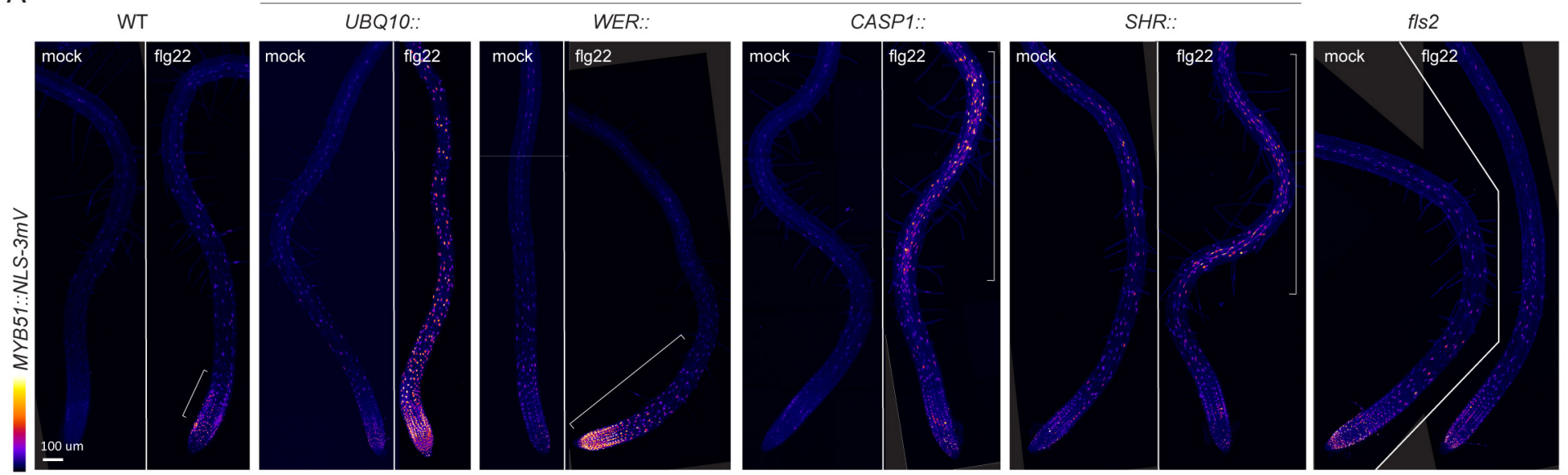

B

WT

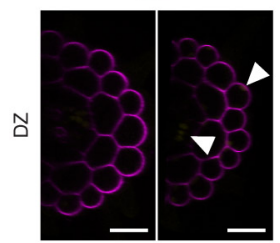

UBQ10 :FLS2-GFP
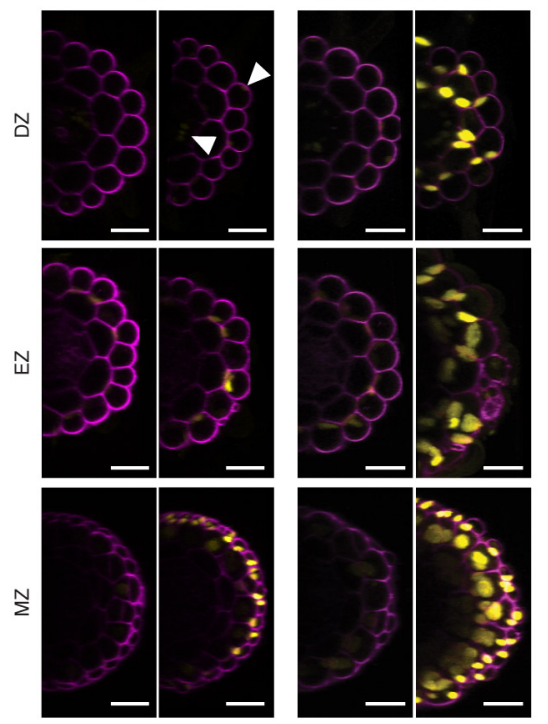

Cell wall fls2

C
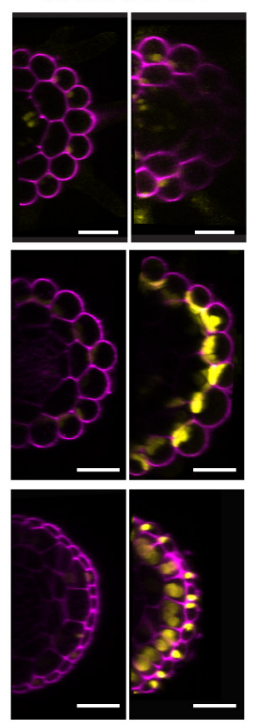
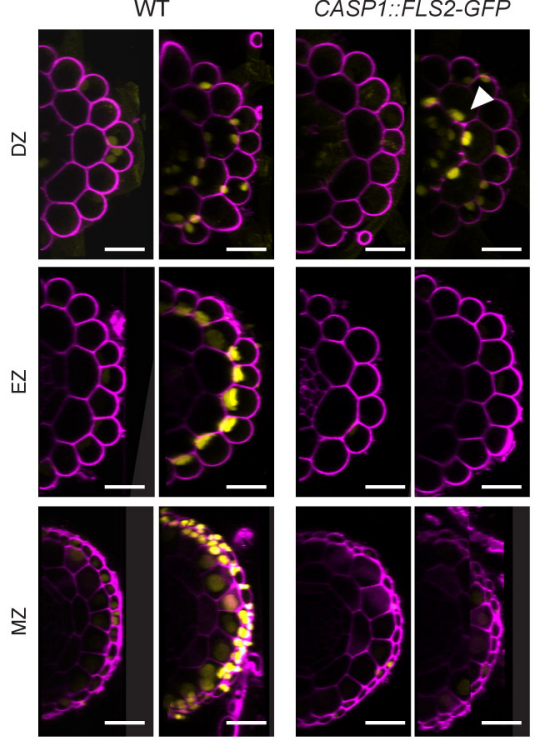

Cell wall fls2
SHR $\because F L S 2-G F P$
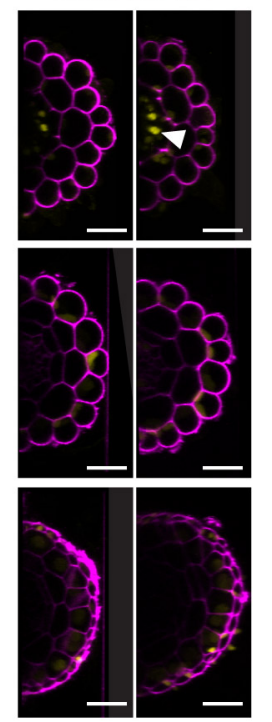

fls2
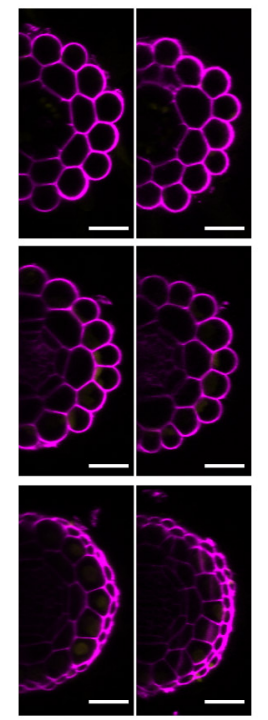

D WT UBQ10::FLS2-GFP fls2 WER::FLS2-GFP fls2 CASP1::FLS2-GFP fls2 SHR::FLS2-GFP fls2 fls2 mock flg22 flgeck

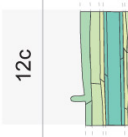

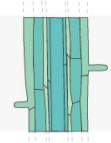
啉 If
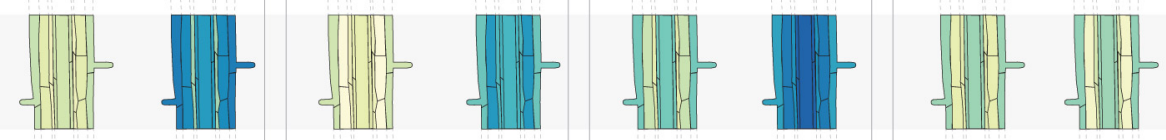

บै HAH HED HAD
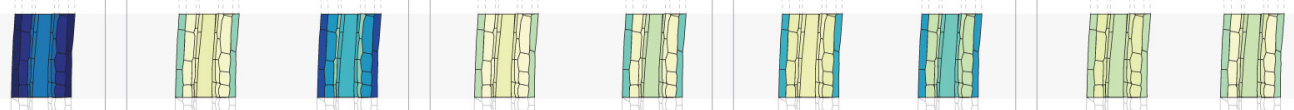

N
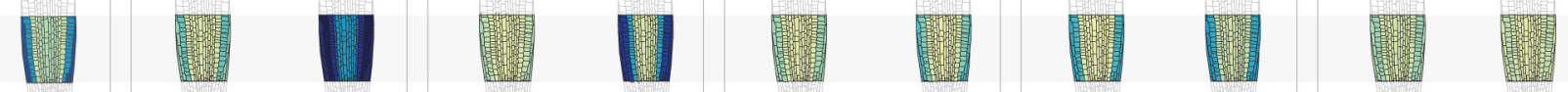

$\log 10$ (Mean Intensity) 


\section{Supplemental Figure S3. MYB51 marker is induced non cell-autonomously by flg22 treatment}

(A) Overview of MYB51::NLS-3mVenus response to $1 \mu \mathrm{M}$ flg22 after 1 day in different prom::FLS2-GFP fls2 lines. MYB51 zone of responsiveness follows FLS2 expression pattern. Tile scan images were taken with similar settings. Settings are always identical between mock and corresponding flg22 treatment.

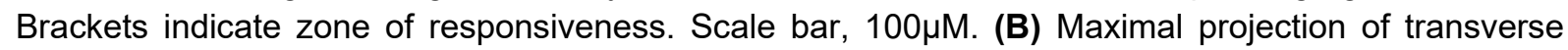
sections views of MYB51 expression pattern in UBQ10:: and WER::FLS2-GFP fls2 compared to WT shown for meristematic zone (MZ), elongation zone (EZ) and differentiated zone (DZ, 30 cells after start of elongation). Seedlings were treated for $24 \mathrm{~h}$ with $1 \mu \mathrm{M}$ flg22. Nuclear localized mVenus signal (yellow) was co-displayed with propidium iodide cell wall marker ( $\mathrm{PI}$, purple). Images were taken with similar settings, while corresponding mock and flg22 treatment pictures for each zone separately have identical parameters. Pictures were acquired with low gain compare to Fig.S2C due to strong average intensity of UBQ10:: and WER::FLS2-GFP fls2 responses, explaining the faint signal in WT (white arrowheads). Scale bar, $25 \mu \mathrm{m}$. (C) Maximal projection of transverse sections views of MYB51::NLS-3mVenus expression in CASP1:: and SHR::FLS2 as well as WT and fls2. MYB51 expression pattern stay conserved (epidermis-cortex-stele), but intensity is increased in neighbourhood of cells expressing FLS2, such as in cortex in CASP1::FLS2-GFP fls2 or stele in SHR::FLS2-GFP fls2 (white arrowheads). Imaged were acquired as Fig.S2B., with similar settings between genotypes, while corresponding mock and flg22 treatment pictures have identical parameters. Due to lower average signal intensity, pictures were acquired with increased gain compare to Fig.2B. Scale bar, $25 \mu \mathrm{M}$. (D) Quantitative map of MYB51::NLS-3mVenus responses inferred from tissue-specific quantification after $24 \mathrm{~h}$ treatment with $1 \mu \mathrm{M}$ flg22. Nuclear signals were quantified in ROI delimited with UBQ10::NLSmTurquoises2 for all tissue-specific promoter lines, while wild-type (WT) signal was quantified with UBQ10::NLS-tdTomato marker. Mean intensity is comparable between prom::FLS2-GFP fls2 lines but not to wild-type. Note the constitutive signal present in untreated seedlings. 


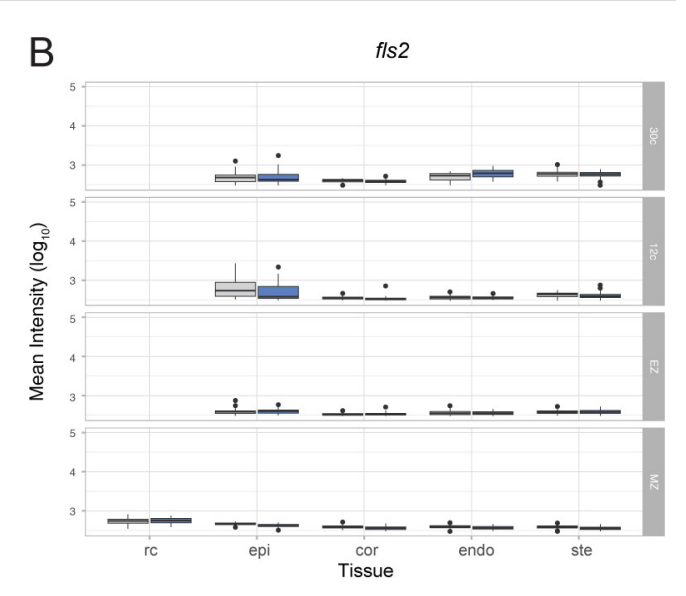

A

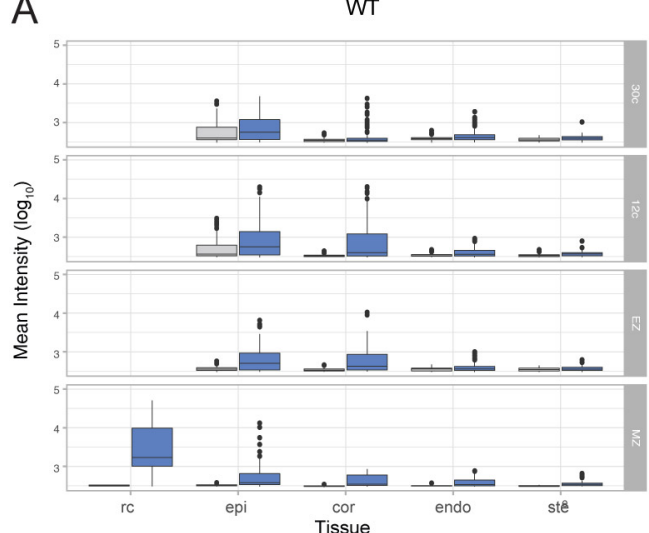

C

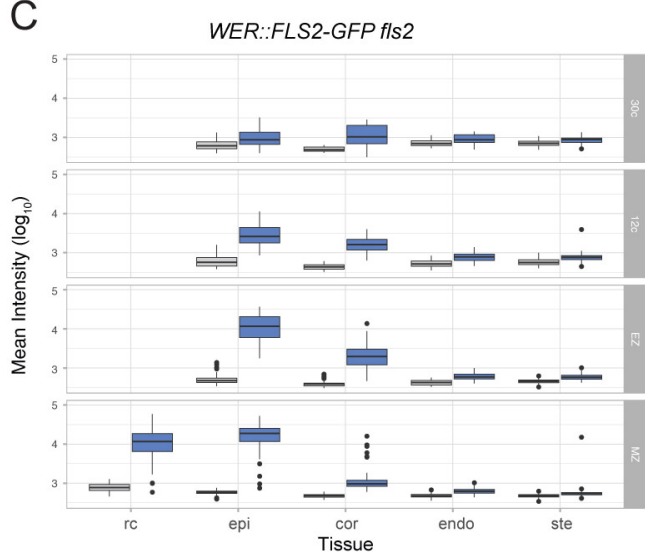

E

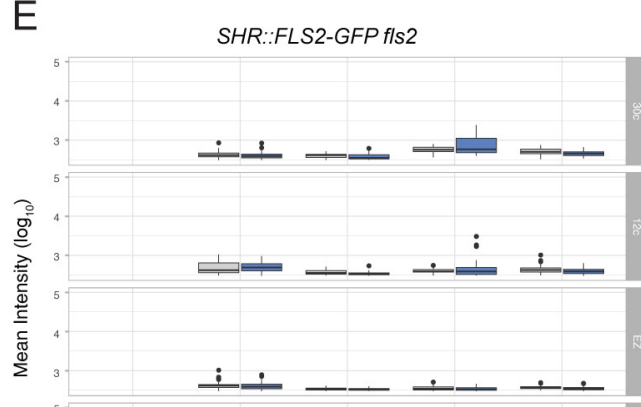

$\div+\div \underset{\text { rc }}{-}+\underset{\substack{\text { cor } \\ \text { Tissue }}}{-}+\frac{1 \text { endo }}{-}+\frac{}{\text { ste }}$
D

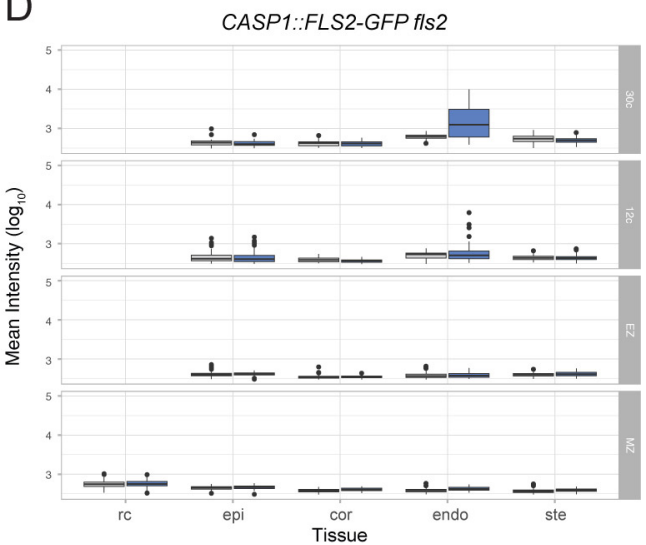

F

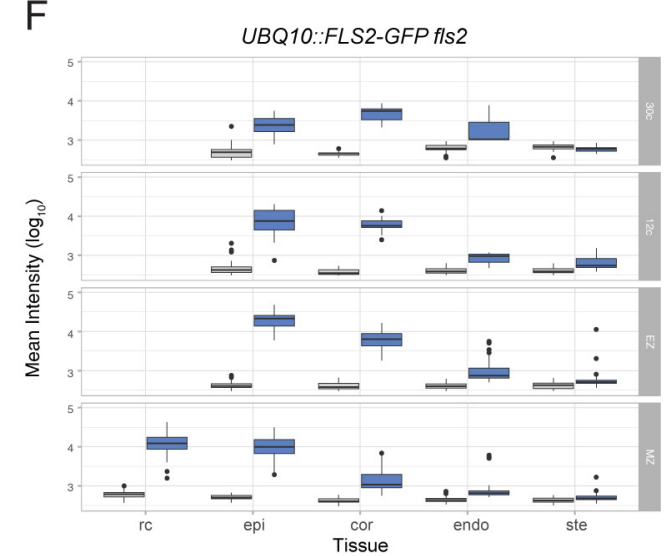

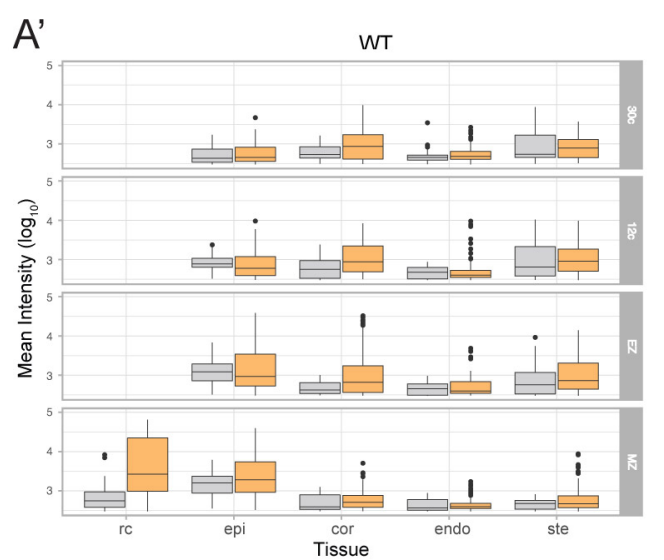

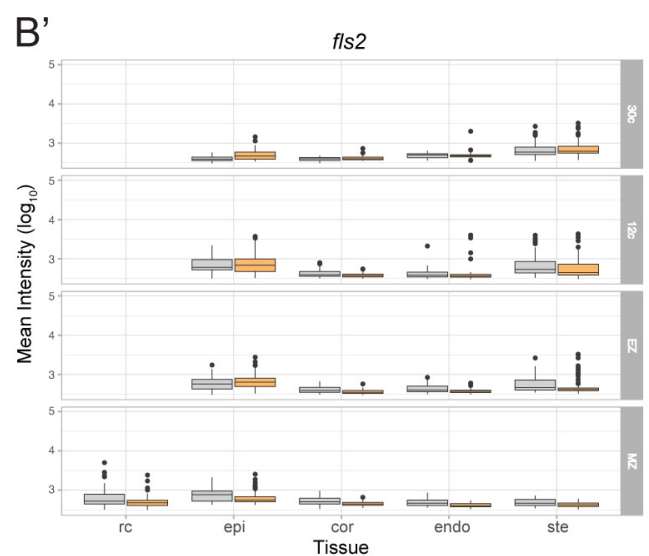

C

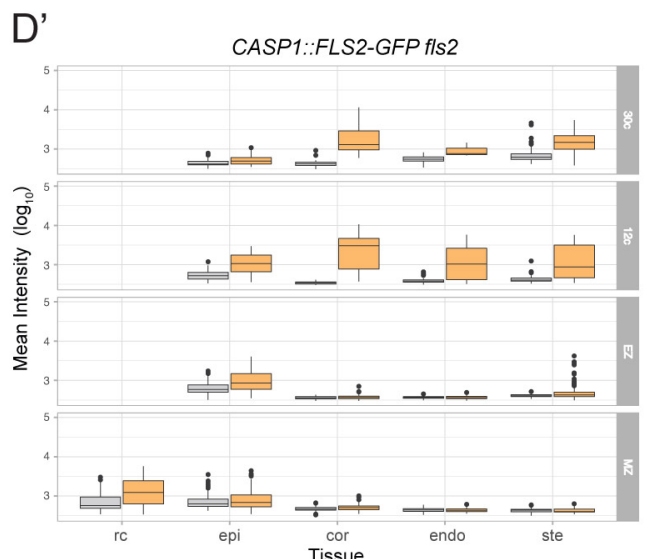

$E^{\prime}$

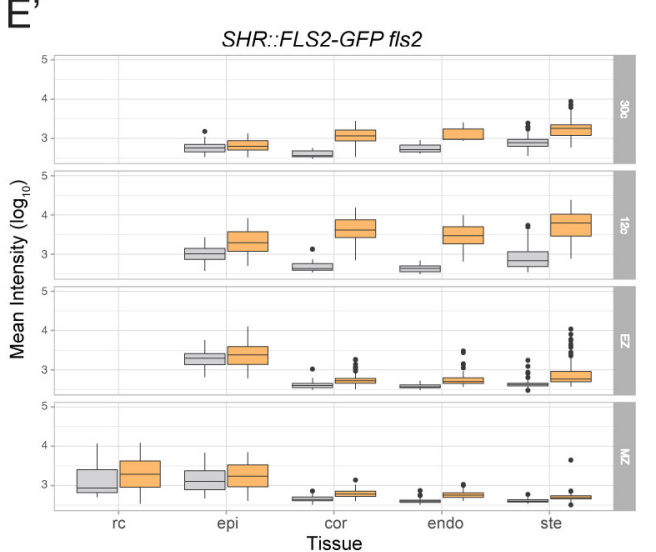




\section{Supplemental Figure S4. PER5 and MYB51 tissue-specific quantification values}

Boxplots for mean intensity of PER5::NLS-3mVenus (A-F) and MYB51::NLS-3mVenus (A'-F') marker calculated from tissue-specific nuclear signals for (A) wild-type plants, (B) fls2 mutant, (C) WER::FLS2-GFP fls2, (D) CASP1::FLS2-GFP fIs2, (E) SHR::FLS2-GFP fls2 and (F) UBQ10::FLS2GFP fls2. Boxplot centre represents the median. MZ, meristematic zone; EZ, elongation zone; $15 \mathrm{c}, 15$ cells after onset of elongation; 30c, 30 cells after onset of elongation; rc, root cap; epi, epidermis; cor, cortex; endo, endodermis; ste, stele. 
A
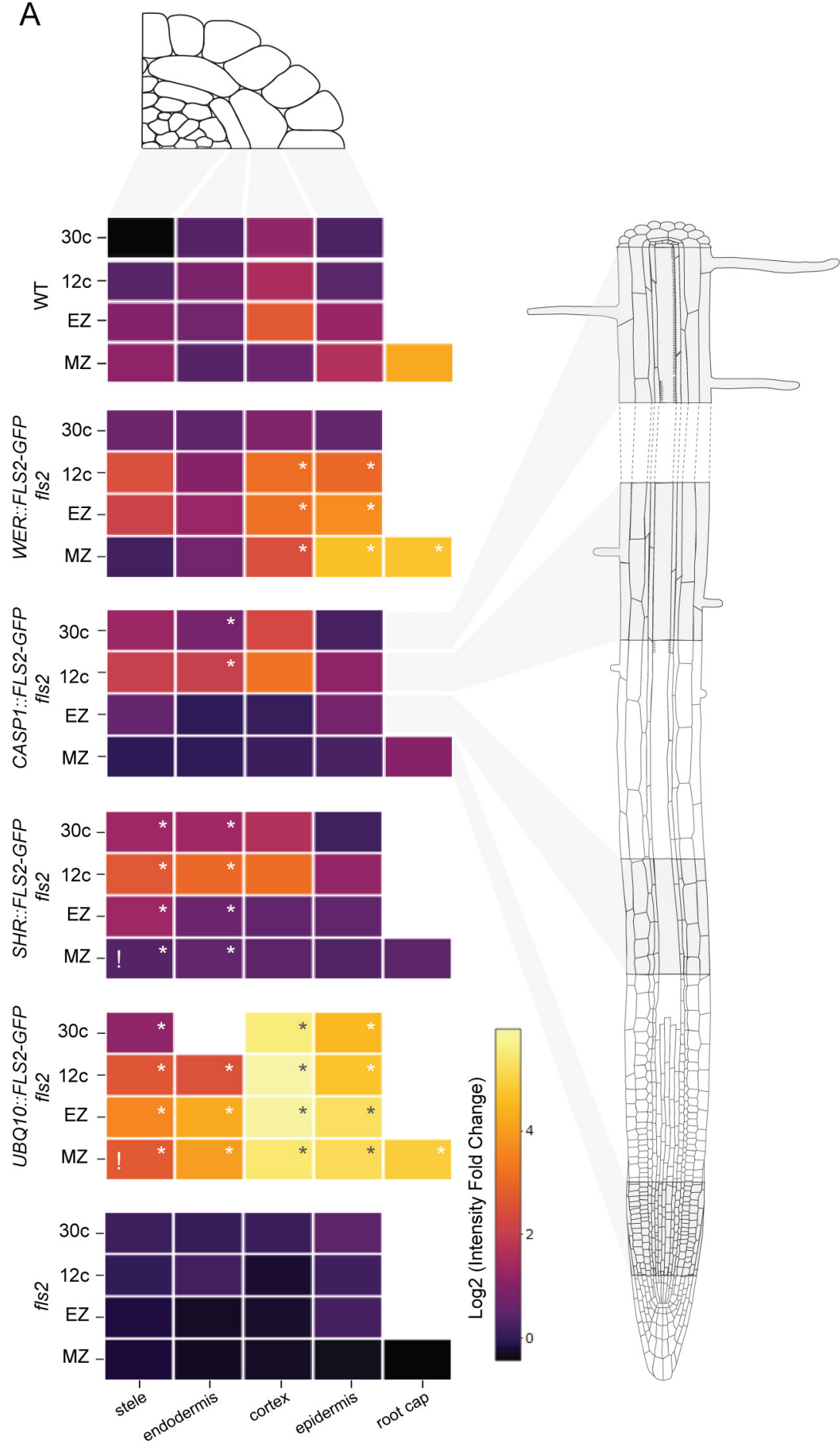

\section{Supplemental Figure S5. Tissue-specific quantification of MYB51 fold change}

$\log _{2}$ transformed fold change of intensity of MYB51::NLS-3mVenus in WT, fls2 and the different prom::FLS2-GFP fls2 lines. Pattern of induction of MYB51 changed between the different lines but increased signal is not restricted to tissue expressing FLS2 (stars). Note that MYB51 can be induced in the stellar meristem in UBQ10::FLS2-GFP fIs2 but not in SHR::FLS2-GFP fIs2 (!). MZ, meristematic zone; EZ, elongation zone; 15c, 15 cells after onset of elongation; 30c, 30 cells after onset of elongation; rc, root cap; epi, epidermis; cor, cortex; endo, endodermis; ste, stele. 
A

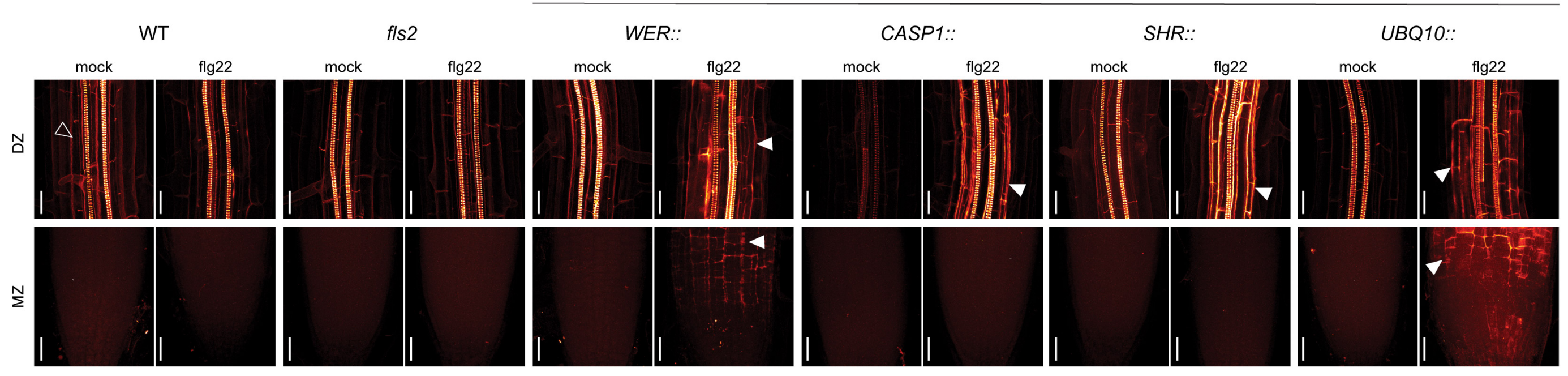

B
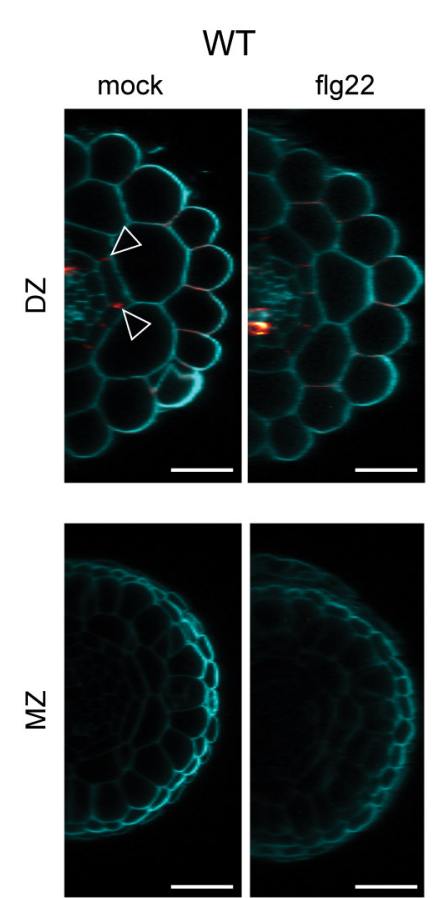
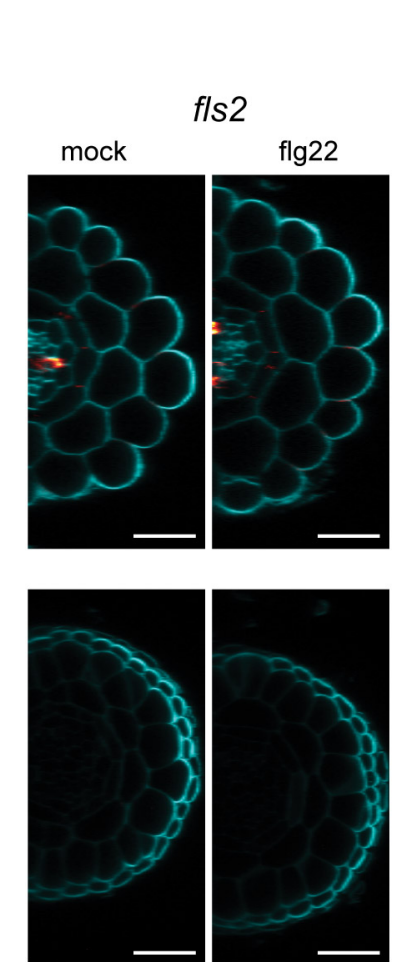

prom::FLS2-GFP fls2
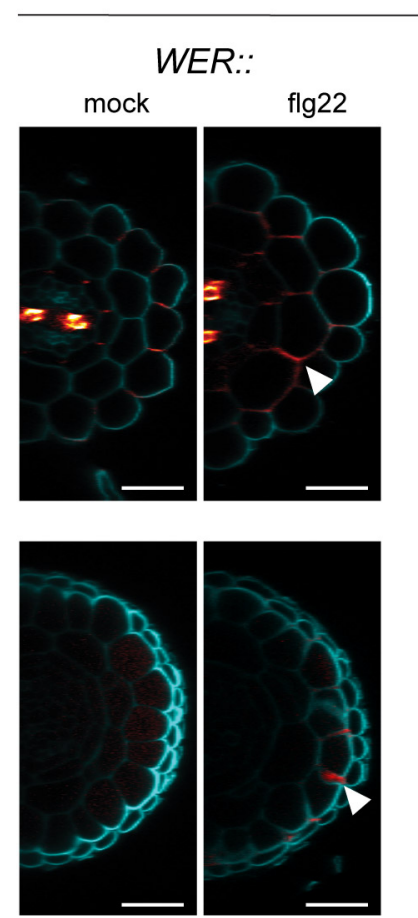
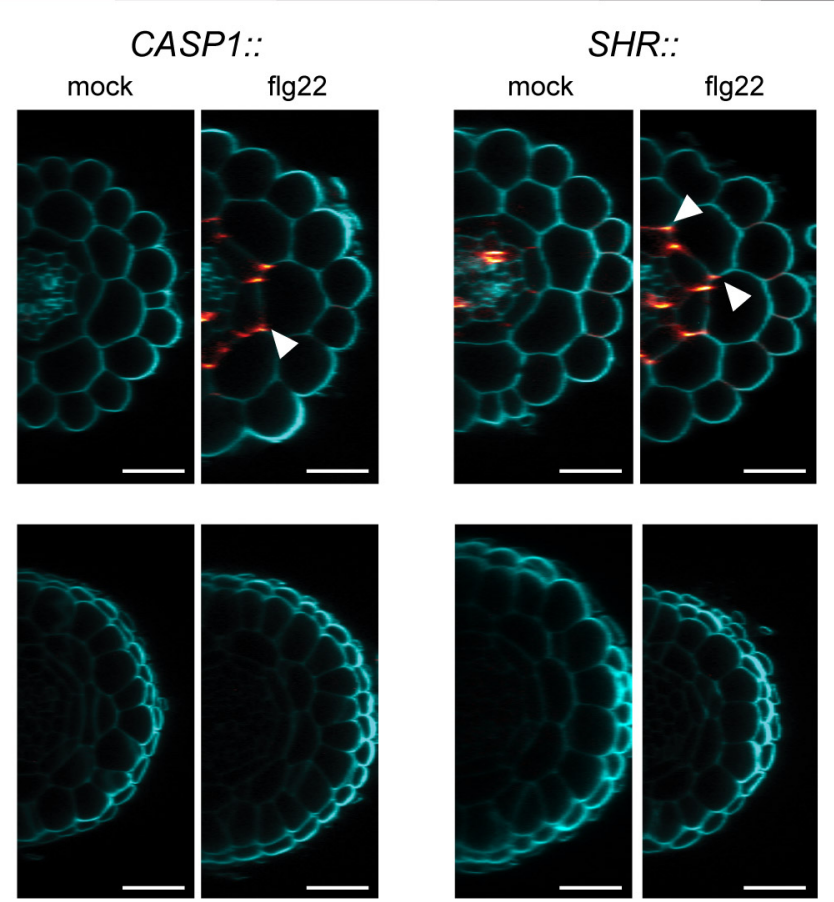
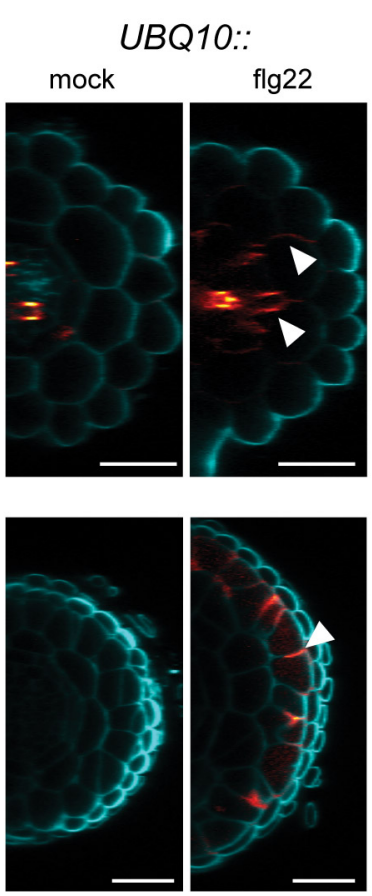


\section{Supplemental Figure S6. Lignin deposition is a cell-autonomous process}

(A) Maximum projection showing lignin deposition stained by basic fuchsin in the meristematic zone (MZ) and the differentiated zone (DZ) of the different prom::FLS2-GFP fls2 lines after 1 day treatment with $1 \mu \mathrm{M}$ flg22. While neither wild-type nor fls2 roots show lignin deposition outside of the xylem and the endodermal Casparian strip barrier, WER:: and UBQ10::FLS2-GFP fls2 lines deposit lignin in both $\mathrm{MZ}$ and DZ. In contrast, CASP1:: and SHR::FLS2-GFP fls2 lignified heavily the DZ only. Black arrowheads, Casparian strip. White arrowheads, ectopic lignin deposition. Scale bar, $25 \mu \mathrm{M}$. (B) Cross section of z-stack presented in (A). Cell wall stained with calcofluor (blue) is co-visualized with lignin stained with basic fuchsin (red). WER::FLS2-GFP expression drives lignin deposition between cortex and epidermal cells in $D Z$, and between epidermal cells and root cap in MZ. This pattern is also observed in UBQ10::FLS2, but extends to cortex and endodermis in DZ. Both CASP1:: and SHR:: deposit lignin ectopically between cortex and endodermal cells after flg22 treatment. White arrowheads, ectopic lignin. Black arrowheads, Casparian strip. Scale bar, $20 \mu \mathrm{M}$. 
bioRxiv preprint doi: https://doi.org/10.1101/2020.08.03.233817; this version posted August 3, 2020. The copyright holder for this preprint (which was not certified by peer review) is the author/funder. All rights reserved. No reuse allowed without permission.

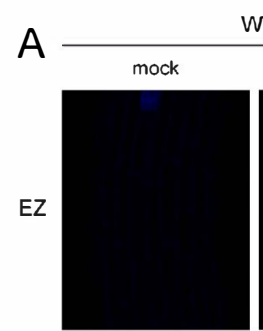

WT
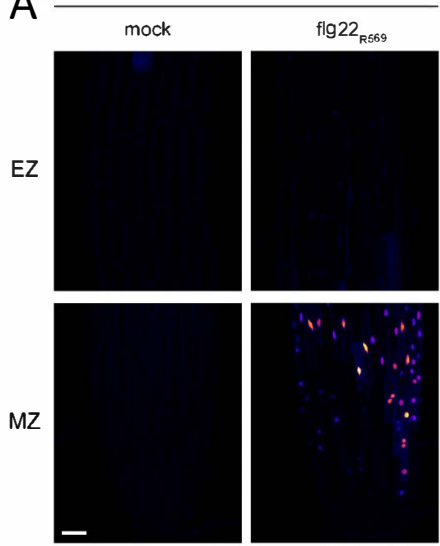

PER5::NLS-3mV

C

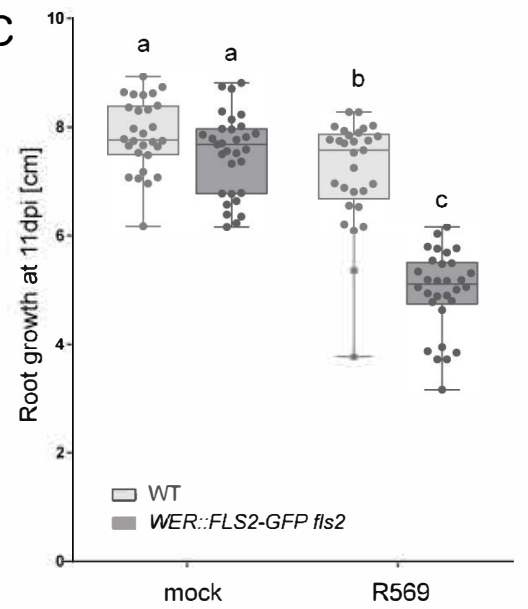

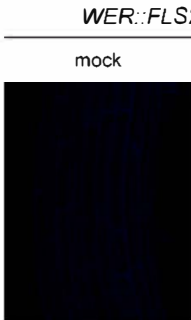
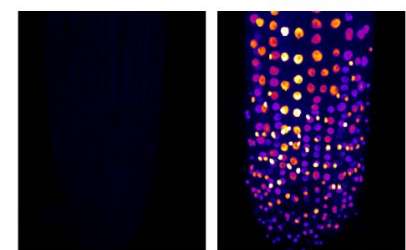

$\mathrm{D}$

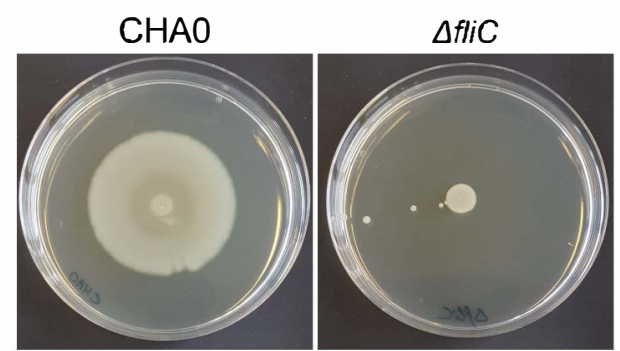

B

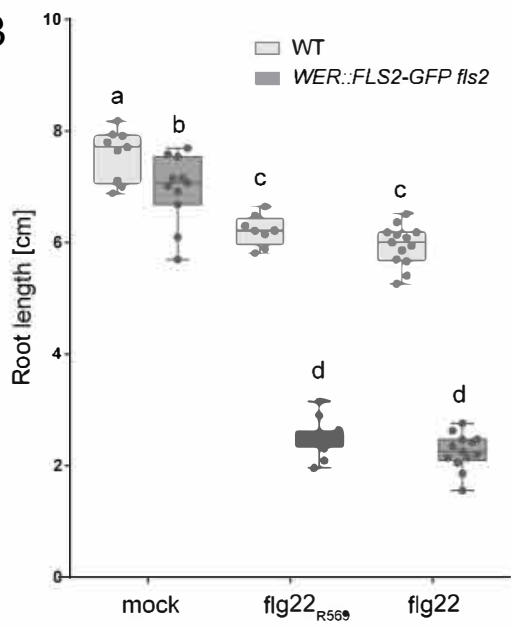

\section{Supplemental Figure S7. Flg22 from bacterial isolate Pseudomonas R569 is recognized by WER::FLS2}

(A) flg22R569 triggers a strong induction of PER5::NLS-3mVenus marker (Fire LUT) on WER::FLS2-GFP fls2 compared to wild-type plant, but the detection is abolished in the fls2 mutant. Maximum projection of z-stacks imaging meristematic $(\mathrm{MZ})$ and elongation $(E Z)$ zones treated for 1 day with $1 \mathrm{uM}$ flg22 $2_{\mathrm{R} 569}$. Acquisition done with identical settings. Scale bar, $25 \mu \mathrm{m}$. (B) flg22 $2_{\mathrm{R} 569}$ inhibits root growth weakly on wild-type (WT) and strongly on WER::FLS2-GFP fls2 in the same extent than commercial flg22 for P.aeruginosa. Seedlings were transferred for 7 days on plates containing $1 \mathrm{uM}$ flg22, flg22 $2_{\mathrm{R} 569}$ or mock. Boxplot centre represents the median. Different letters indicate statistically significant difference $(p<0.05)$ between means by 2 -ways ANOVA and Tukey's multiple comparison tests. (C) Bacterial isolate R569 induces stronger root growth inhibition on wild-type seedlings (WT) than on WER::FLS2-GFP fls2. Replicate carried out in Cologne with different growth conditions (see material and methods). Five-days old seedlings were transferred for 11 days on plate containing bacteria at a concentration of $\mathrm{OD}_{600}=0.01$. Boxplot centre represents the median. Different letters indicate statistically significant difference $(p<0.05)$ between means by ANOVA and Tukey's multiple comparison tests. (D) Motility assay for CHAO and its $\triangle$ fliC mutant. 